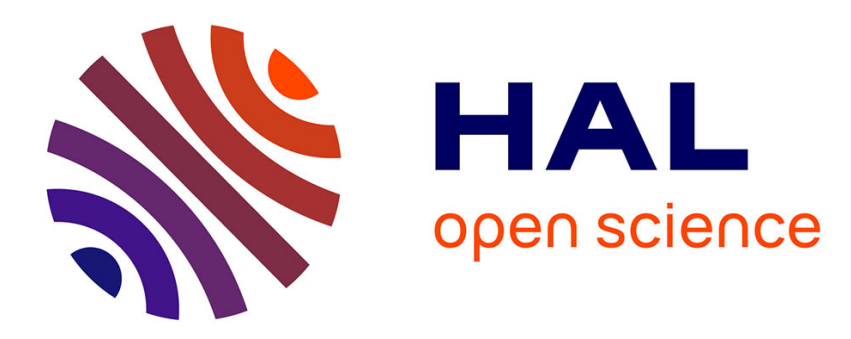

\title{
A note on the crowding-out of investment by public spending \\ Olivier Cardi
}

\section{- To cite this version:}

Olivier Cardi. A note on the crowding-out of investment by public spending. 2009. hal-00420132

\section{HAL Id: hal-00420132 \\ https://hal.science/hal-00420132}

Preprint submitted on 28 Sep 2009

HAL is a multi-disciplinary open access archive for the deposit and dissemination of scientific research documents, whether they are published or not. The documents may come from teaching and research institutions in France or abroad, or from public or private research centers.
L'archive ouverte pluridisciplinaire HAL, est destinée au dépôt et à la diffusion de documents scientifiques de niveau recherche, publiés ou non, émanant des établissements d'enseignement et de recherche français ou étrangers, des laboratoires publics ou privés. 


\title{
ECOLE POLYTECHNIQUE
}

CENTRE NATIONAL DE LA RECHERCHE SCIENTIFIQUE

\section{A NOTE ON THE CROWDING-OUT OF INVESTMENT BY PUBLIC SPENDING}

\author{
Olivier CARDI
}

June 2009

Cahier $n^{\circ}$ 2009-38

\section{DEPARTEMENT D'ECONOMIE}

Route de Saclay

91128 PALAISEAU CEDEX

(33) 169333033

http://www.enseignement.polytechnique.fr/economie/

mailto:chantal.poujouly@polytechnique.edu 


\title{
A NOTE ON THE CROWDING-OUT OF INVESTMENT BY PUBLIC SPENDING ${ }^{1}$
}

\author{
Olivier CARDI ${ }^{1}$ \\ ERMES, Université Panthéon-Assas Paris 2 \\ Département d'Économie, Ecole Polytechnique
}

1st June 2009

\begin{abstract}
One of the most prominent and consistent findings of the recent empirical literature on fiscal policy is that investment expenditure is crowded-out by public spending in the short-run. In this contribution, we address this empirical fact using a dynamic general equilibrium model and show that the introduction of a habit-forming behavior plays a major role in accommodating the observed negative relationship between investment and government expenditure. Our numerical experiments point out the role of consumption inertia in determining the reactions of the open economy: as habit persistence gets stronger, a fiscal expansion crowds-out real consumption by a smaller amount and investment by a larger one, while the current account enters into a greater deficit.
\end{abstract}

Keywords: Investment; Habit Formation; Current Account; Fiscal Expansion JEL Classification: F41; F32; E21; E62

\footnotetext{
${ }^{1}$ This paper is a shortened version of a previous manuscript available at http://ermes.u-paris2.fr/ doctrav08.htm. Constructive suggestions by Peter Claeys, Stefan Schubert, Partha Sen and Michael Visser are gratefully acknowledged. I am especially grateful to Luisito Bertinelli and Eric Strobl for their detailed comments. An earlier version of this paper was presented to the 62nd European Meeting of Econometric Society held in Budapest, 27-31st August 2007, the seminar Economics-Finance held at the University of Luxembourg, 26th September 2007, the 12th Conference Theories and Methods of Macroeconomics, 17-18th January 2008, and the 14th Conference on Computing in Economics and Finance, 26-28th June 2008, held in Paris and has benefited from helpful comments of participants. Finally, I would like to thank Cem Karayalçin and the editor William Barnett for very constructive suggestions and remarks which improve both content and exposition of a previous draft. All remaining shortcomings are ours.

${ }^{2}$ Correspondence: Département d'Économie, École Polytechnique 91128 Palaiseau Cedex, France, Phone: +3316933 30 38, Fax: +331693334 27. E-mail: olivier.cardi@u-paris2.fr.
} 


\section{Introduction}

One of the most prominent and consistent findings of the empirical literature related to the macroeconomic effects of fiscal shocks is that investment expenditure is crowded-out by public spending in the short-run (see e. g. Blanchard and Perotti [2002], Mountford and Uhlig [2005], Perotti [2005], Afonso and Sousa [2009]). However, the theoretical explanation of this empirical fact remains unsatisfactory. While standard Keynesian theory would predict that crowding-out occurs through increases in interest rates, Mountford and Uhlig [2005] find that empirically "government spending shocks crowd out both residential and non-residential investment without causing interest rates to rise". Similarly, although the neo-classical Real Business Cycle literature has the advantage of being based on sound theoretical foundations, as exemplified by Baxter and King [1993], its predictions are also at odds with empirical facts as we shall discuss below. In this paper, in contrast, we are able to provide a theoretical explanation of the crowding-out of investment by relaxing the assumption of time separability in utility within a Baxter and King type framework.

Open economy versions of Baxter and King's [1993] model considering standard preferences find that consumption reacts strongly to a fiscal shock, investment is crowded-in, and the current account enters in deficit due to capital accumulation (see e. g., Karayalçin [1999]). These conclusions come as a result of the assumption of time separable preferences implying that the representative household chooses a perfect smoothing temporal path for real expenditure. Thus, after a balanced-budget fiscal expansion, consumption overreacts to the fall in real disposable income which in turn releases enough resources for capital accumulation. Since saving behavior plays a minor role, the investment boom drives the current account into deficit. However, recent empirical results cast doubt over these three conclusions. First, according to estimates documented by Perotti [2005], Afonso and Sousa [2009], a fiscal shock crowds-out investment instead of boosting capital accumulation. Second, empirical evidence provided by Mountford and Uhlig [2005], and corroborated by Afonso and Sousa [2009], reveals that private consumption does not change significantly after a fiscal expansion. This finding suggests 
that consumption weakly reacts in the short-run, and thereby that the marginal propensity to consume displays a small value. Third, estimates by Chinn and Prasad [2003] show that an increase in fiscal deficit leads to a higher current account deficit in both developing and industrialized countries. More specifically, empirical evidence documented by Freund [2005] reveals that short-run current account deficits episodes are more associated with a decline in savings than with an increase in investment.

Here we show that the introduction of a habit index into the utility function helps to improve the predictive power of the Baxter and King [1993] model by modifying the chain of events following a fiscal expansion. More precisely, higher fiscal spending financed by lump-sum taxes reduces private wealth and induces agents to cut their real expenditure. However, since agents wish to sustain their original standard of living, consumption reacts smoothly and savings fall. At the same time, consumption inertia implies that the reduction in private demand is not large enough to offset additional public spending such that investment is crowded-out. Whatever the degree of habit persistence, the drop in savings always more than offsets the decline in investment and thereby the open country experiences an initial current account deficit.

The remainder of the paper is organized as follows. In section 2, we develop a novel twogood open economy model with habit formation. The macroeconomic equilibrium is analyzed in section 3. Section 4 discusses the effects of a permanent fiscal expansion and provides a sensitivity analysis which highlights the quantitative role of habit persistence. Section 5 concludes.

\section{An Open Economy Model with Habit Formation}

We consider a semi-small open economy that is populated by a large number of identical households and firms that have perfect foresight and live forever. The country is assumed to be semi-small in the sense that it is price-taker in international capital markets (i. e. , the world interest rate $r^{\star}$ is exogenous) but is large enough on world good markets to influence the price of its export goods. 


\subsection{Households}

At each instant the representative household consumes domestic and foreign goods denoted by $c^{D}$ and $c^{F}$, respectively, which are aggregated by means of a CES function:

$$
c=\left[\varphi^{\frac{1}{\phi}}\left(c^{D}\right)^{\frac{\phi-1}{\phi}}+(1-\varphi)^{\frac{1}{\phi}}\left(c^{F}\right)^{\frac{\phi-1}{\phi}}\right]^{\frac{\phi}{\phi-1}}
$$

where $\varphi$ is the weight of the domestic good in the overall consumption bundle $(0<\varphi<1)$ and $\phi$ corresponds to the intratemporal elasticity of substitution between domestic and foreign consumption goods. In line with Carroll, Overland and Weil [2000], agents care about both current and past real consumption, denoted by $c$ and $s$ respectively, in deriving utility. The representative household maximizes the following objective function:

$$
U=\int_{0}^{\infty} \frac{1}{1-\epsilon}\left[\frac{c}{(s)^{\gamma}}\right]^{1-\epsilon} e^{-\beta t} \mathrm{~d} t,=\int_{0}^{\infty} \frac{1}{1-\epsilon}\left[(c)^{1-\gamma}\left(\frac{c}{s}\right)^{\gamma}\right]^{1-\epsilon} e^{-\beta t} \mathrm{~d} t
$$

where $\beta$ is the consumer's discount rate, $\epsilon>0$ corresponds to the coefficient of relative risk aversion, and $\gamma>0$ stands for the weight attached to habits in utility. According to (2), agents derive utility from a geometric weighted average of absolute and relative consumption where $\gamma$ is the weight of relative consumption. If $\gamma=0$, the comparison of real consumption to the reference stock turns out to be irrelevant and the case of time separability in preferences is obtained. If $\gamma>0$, then agents care about their relative consumption in deriving utility which influences their consumption choices. They take into account that for a given change in their current consumption, the consecutive adjustment of habits influences the marginal utility of future consumption which modifies the intertemporal marginal rate of substitution and thereby the allocation of real expenditure.

The habit stock denoted by $s(t)$ is defined as a distributed lag on past real consumption:

$$
s(t)=\sigma \int_{-\infty}^{t} c(\tau) e^{-\sigma(t-\tau)} \mathrm{d} \tau
$$

where the parameter $\sigma$ indexes the relative weight of recent consumption in determining the reference stock $s$. Differentiating equation (3) w. r. t. time gives the law of motion of habit stock:

$$
\dot{s}(t)=\sigma[c(t)-s(t)]
$$


Intuitively, the larger is $\sigma$, the greater is the weight of consumption in the recent past in determining the stock of habits, and the faster the reference stock adjusts to current expenditure. By noting that habits coincide with real consumption in the long run, setting $c=s$ into the iso-elastic function (2) leads to a long-run intertemporal elasticity of substitution (IES) denoted by $\nu=\frac{1}{[\gamma+\epsilon(1-\gamma)]}$. Since the long-term IES under time non separable preferences is higher than the standard IES $1 / \epsilon$ as long as the $\epsilon>1$, the steady-state change of consumption is magnified. ${ }^{1}$

Households supply inelastically one unit of labor services and hold physical capital stock for which they receive wage rate $w(t)$ and the capital rental rate respectively. In addition, they accumulate internationally traded bonds holding, $b(t)$, that yields net interest rate earnings $r^{\star} b(t)$, expressed in terms of the foreign good. Denoting lump-sum taxes by $T$ and the consumption price index by $p_{c}$, the flow budget constraint is equal to households' real disposable income less absorption:

$$
\dot{b}(t)=\frac{1}{p(t)}\left\{r^{\star} p(t) b(t)+\left(r^{K}(t)+\delta_{K}\right) k(t)+w(t)-T-p_{c} c(t)-I(t)\right\},
$$

where gross investment $I$ is given by:

$$
I(t)=\dot{k}(t)+\delta_{K} k(t)
$$

where $\delta_{K} \geq 0$ is a fixed depreciation rate.

Since $c($.$) is homothetic, the household's maximization problem can be decomposed into two$ stages. In the first stage, the representative household chooses the optimal levels of consumption as well as investment in physical capital and foreign bonds. The cost-minimizing allocation of real expenditure between domestic goods and imports can be decided at the second stage which yields: $c^{D}=\left(1-\alpha_{c}\right) p_{c} c$ and $p c^{F}=\alpha_{c} p_{c} c$, with $\alpha_{c}$ the share of foreign goods in consumption expenditure.

\section{$2.2 \quad$ Firms}

A large number of identical and perfectly competitive firms produce a final good which can be consumed domestically, invested, or exported. They use physical capital $k$ and labor $n$, 
according to a constant returns to scale production function, $Y=F(k, n)$, which is assumed to have the usual neoclassical properties of positive and diminishing marginal products. Profit maximization gives the standard first order conditions according to which capital marginal product is equal to the capital rental rate, i. e. , $F_{k}=r^{K}+\delta_{K}$, and labor marginal product is equal to the the wage rate, i. e., $F_{n}=w .^{2}$

\subsection{Government}

The final agent in the economy is the government who finances public spending on the domestic good $g^{D}$ and imports $g^{F}$ by raising lump-sum taxes $T$ in accordance with the balanced budget condition: $g^{D}+p g^{F}=T$.

\section{Macroeconomic Equilibrium}

\section{First-Order Conditions}

Denoting by $\lambda$ and $\xi$ the shadow prices of wealth and habits, the macroeconomic equilibrium is defined by the following set of equations:

$$
\begin{gathered}
c^{-\epsilon} s^{-\gamma(1-\epsilon)}+\sigma \xi=p_{c}(p) \lambda / p, \\
\dot{\lambda}=\lambda\left(\beta-r^{\star}\right), \\
\dot{\xi}=(\beta+\sigma) \xi+\gamma c^{1-\epsilon} s^{-[\gamma(1-\epsilon)+1]}, \\
\dot{p}=p\left(r^{K}-r^{\star}\right), \quad \text { with } \quad r^{K}=F_{k}-\delta_{K},
\end{gathered}
$$

and dynamic equations (4), (5), (6), and appropriate transversality conditions. In order to generate an interior solution, we require that $\beta=r^{\star}$. This standard assumption made in the literature implies that the marginal utility of wealth, $\lambda$, will undergo a discrete jump when individuals receive new information and must remain constant over time from thereon.

The dynamic efficiency condition (7d) asserts that the real exchange rate adjusts so as to equalize the rate of return on domestic physical capital net of capital depreciation, i. e. , $r^{K}$, to the real rate of return on traded bonds, i. e. , $r^{\star}+\dot{p} / p$. The real exchange rate dynamics 
influence in turn the consumption temporal path by affecting the consumption-based real interest rate. The law of motion for real consumption can be expressed in an interpretable form by using static and dynamic optimality conditions (7a) and (7c). Differentiating (7a) with respect to time, eliminating the shadow value of habits $\xi(t)$ and substituting $(7 \mathrm{c})$, we obtain the dynamic equation for $c$ :

$$
\frac{\dot{c}}{c}=\frac{1}{\epsilon}\left[1+\sigma \xi c^{\epsilon} s^{\gamma(1-\epsilon)}\right]\left[r^{\star}+\left(1-\alpha_{c}\right) \frac{\dot{p}}{p}-\rho(c, s, p)\right] .
$$

Equation (8) restates the consumption Euler equation, which relates the optimal profile of consumption to the difference between the consumption-based real interest rate, $r^{c}=r^{\star}+$ $\left(1-\alpha_{c}\right) \frac{\dot{p}}{p}$, and the rate of time preference, denoted by $\rho(t)$, which is variable over time.

Denoting exports by $X$, we impose a domestic good market clearing condition to fully describe the macroeconomic equilibrium: ${ }^{3}$

$$
F(k)=c^{D}+X(p)+I+g^{D}
$$

where investment adjusts to equalize supply and aggregate demand along the transitional path.

\section{Steady-State}

The steady-state of the economy is obtained by setting $\dot{c}, \dot{s}, \dot{k}, \dot{p}, \dot{b}=0$ and is defined by the following set of equations: ${ }^{4}$

$$
\begin{gathered}
\tilde{c}=\left[\left(\frac{\beta+\sigma}{\beta+\sigma(1-\gamma)}\right) \frac{p_{c} \bar{\lambda}}{\tilde{p}}\right]^{-\frac{1}{\gamma+\epsilon(1-\gamma)}}, \\
F(\tilde{k})=\tilde{c}^{D}+X(\tilde{p})+g^{D}+\delta_{K} \tilde{k}, \\
r^{\star} \tilde{b}+X(\tilde{p}) / \tilde{p}-\tilde{c}^{F}-g^{F}=0, \\
\left(\tilde{b}-b_{0}\right)=\Phi_{1}\left(\tilde{k}-k_{0}\right)+\Phi_{2}\left(\tilde{s}-s_{0}\right),
\end{gathered}
$$

where the stock of habits $\tilde{s}$ must coincide with real consumption once the economy reaches the steady-state. Equation (10b) restates that the production of domestic goods must be exactly outweighed by a demand counterpart. Equation (10c) implies that in the steady-state equilibrium, net foreign interest receipts from traded bonds holding must be exactly matched by net exports. Finally, equation (10d) represents the linearized version of the intertermporal 
solvency constraint of the open economy according to which the net foreign asset position is determined by both investment and consumption decisions. ${ }^{5}$

Equation (10a) determines the long-run level of real consumption. Whenever the relative weight attributed to habit stock $\gamma$ is positive, the long-run rate of change of $c$ is influenced by the long-term IES with time non separable preferences $\nu$, which is higher than the inverse of relative risk aversion $1 / \epsilon$. Hence, after a reduction of private wealth, habit-forming consumers experience a larger steady-state fall in consumption than a household displaying standard preferences. More precisely, the closer to unity $\gamma$ is, the larger is $\nu$ and the greater is the long-run decrease in $c$ for a given reduction in private wealth. The explanation is that as $\gamma$ gets closer to unity, households cut their real expenditure by a smaller amount in the short-run. This behavior has a counterpart: they must reduce their real consumption by a greater amount in the long-run as they decumulate financial wealth over the transition. Finally, a greater trade openness softens the drop of real consumption by moderating the decrease in private wealth measured in terms of the domestic good.

\section{Fiscal Expansion and Adjustment of the Open Economy}

In this section, we investigate the steady-state and dynamic effects of a permanent increase in government spending on the domestic good $g^{D}$.

\subsection{Benchmark Parametrization}

We briefly discuss the calibration by starting first with the parameters that are kept fixed throughout the simulations. The parameter $\phi$ is set to 1.5 . The parameter $\varphi$ is set such that the share of consumption of imported goods is approximately $19 \%$ of total expenditure (see, e. g. , Corsetti and Müller [2006]). The world interest rate $r^{\star}$, is set at 4\%. Exports are assumed to take a power form: $X(p)=\gamma_{X} p^{\nu_{X}}$ with $\gamma_{X}>0$ a scaling parameter. The real exchange rate elasticity of exports denoted by $\nu_{X}$ is set to 0.8 . Government spending as a share of GDP $g / Y$ is assumed to be $20 \%$. The import content of government expenditure is 
set to $10 \%$ (see Corsetti and Müller [2006]). The production function takes a Cobb-Douglas form, $Y=F(k)=k^{\alpha_{K}}$, with an output share of capital income, $\alpha_{K}$, set to 0.35 . Finally, we set $\delta_{K}$ at $5 \%$ so as to be consistent with a ratio investment-GDP roughly equal to $20 \%$.

The critical parameters pertain to the weight of habits in utility, $\gamma$, and the speed of adjustment, $\sigma$, in the determination of the reference stock. We set $\gamma$ at 0.8 in line with empirical estimates documented by Sommer [2007]. The relative-risk aversion parameter $\epsilon$ is set at 2.5 which implies a long-run IES of $\nu=\frac{1}{\gamma+\epsilon(1-\gamma)}=0.77$ falling in the range of 0.5 and 1 of empirical estimates. Finally, we set $\sigma$ at 0.5 .

\subsection{Steady-State Effects}

We begin by examining the steady-state responses of a fiscal expansion, then turn to dynamic effects. Quantitative estimation of the long-term and impact responses of key macroeconomic variables are reported in Tables $1 \mathrm{~A}$ and $1 \mathrm{~B}$ respectively. We consider four alternative scenarios: time preferences $(\gamma=0)$, benchmark parametrization $(\gamma=0.8, \sigma=0.5)$, weak habits $(\gamma=0.3)$, a faster speed of habits $(\sigma=0.9)$, a greater trade openness $(\varphi=0.5){ }^{6}$

Since the increase in government purchases is financed by a rise in lump-sum taxes in order for the budget balanced condition to hold, a permanent fiscal expansion reduces households' after-tax lifetime income. As agents feel poorer, they are induced to cut their real expenditure. Real consumption is depressed further through an additional channel. An expansionary budget policy triggers an excess of demand in the home good market which requires a larger steadystate reduction in consumption. The associated long-term reduction in habits results in a decrease in the stock of foreign assets as agents decumulate financial wealth to sustain their original standard of living in the short-run. Additionally, in an open economy, a rise in $g^{D}$ depresses further private demand through a long-term real exchange rate appreciation which reduces exports.

Table $1 \mathrm{~A}$ provides a sensitivity analysis regarding the long-term effects of a rise in $g^{D}$. In line with theoretical results, as habit persistence in consumption gets stronger, real consumption 
falls by a larger amount. Whereas consumers cut their expenditure by around $-0.8 \%$ of initial GDP if preferences are time separable (i. e. , $\gamma=0$ ), $p_{c} \tilde{c}$ declines by $-0.81 \%$ if habits are weak (i. e., $\gamma=0.3$ ) and by $-0.85 \%$ for the benchmark parametrization (i. e., $\gamma=0.8$ ). Hence, as $\gamma$ gets closer to unity, the long-run IES gets larger such that private consumption drops further in response to the fall in private wealth. Additionally, as trade openness increases, the domestic content of consumption expenditure falls which in turn moderates sizeably the drop in private wealth measured in terms of the domestic good, and thereby softens considerably the reduction of consumption in the long-run.

$<$ Please insert Table 1 about here $>$

\subsection{Dynamic Effects}

\section{Impact Effects}

Without habits, the short-run marginal propensity to consume (MPC) is equal to unity such that at the time when fiscal expansion is implemented, real consumption falls by the same amount than the decline in real disposable income, leaving savings unaffected. In contrast, as long as people pay attention to relative consumption in deriving utility, the short-term MPC turns out to be smaller than unity. Henceforth, real consumption initially falls but by less than in the long-term. The explanation is that, facing a fall in their disposable income due to higher lump-sum taxes, habit-forming consumers are aware that their standard of living will be lower at the steady-state, which drives down the marginal utility of future consumption. At the time the fiscal expansion is implemented, the stock of habits remains fixed. Hence, the marginal utility of current consumption exceeds the marginal utility of future consumption, which in turn provides a strong incentive to reallocate expenditure in the present. The rise in the intertemporal marginal rate of substitution yields a smooth initial response of consumption in line with empirical evidence provided by Mountford and Uhlig [2005] and Afonso and Sousa 
[2009]. For the benchmark parametrization, the first row of Table 1B shows that the cut in real expenditure at time $t=0$ is about 2.5 times smaller than in the long run.

One prominent and consistent findings of the recent empirical literature on fiscal policy is that investment is crowded-out by public spending. As we shall see now, short-run consumption inertia plays a major role in accommodating this empirical fact. To derive the initial response of investment, we differentiate the market-clearing condition for the home good:

$$
\mathrm{d} I(0)=-\Theta \mathrm{d} p(0)-\left(c^{D} / c\right) \mathrm{d} c(0)-\mathrm{d} g^{D}
$$

where $\Theta \equiv \frac{X}{p}\left[\nu_{X}+\frac{c^{D}}{X} \phi \alpha_{c}\right]>0$ captures the impact of a change of the real exchange rate on exports and consumption. Equation (11) shows that the initial reaction of investment is the result of two opposing effects. On the one hand, the fall in private demand (i. e., exports and private consumption) releases resources for capital accumulation (see the first and second terms on the RHS of (11)). On the other hand, higher public purchases withdraw resources from the private sector, which implies the possibility that investment falls in the short-term (see the last term on the RHS of (11)). If preferences are time separable (i. e. , $\gamma=0$ ), investment is unaffected as the economy reaches its new steady-state immediately. In contrast, regardless of the strength of consumption inertia, the short-term decline in private demand is not large enough to compensate for the rise in $g^{D}$. Hence, an excess of demand arises in the home goods market which must be eliminated by a decumulation of physical capital. Interestingly, as can be seen from the last column of Table $1 \mathrm{~B}$, trade openness plays a major role in determining the size of the crowding-out as investment falls by about $-0.1 \%$, which is more than three times smaller than in the benchmark scenario. The reason for this is that as the economy gets more open, the real exchange appreciation crowds-out consumption by a smaller amount and exports by a much larger one.

Finally, from the fourth and fifth rows of Table 1B, the investment rate falls by $0.3 \%$ while savings drops by $0.4 \%$ of initial GDP for the baseline calibration. ${ }^{7}$ Hence, the current account enters into deficit. As expected, the worsening in the net foreign asset position is magnified as trade openness increases due to the dramatic reduction of exports. 
$<$ Please insert Figures 1(a), 1(b) about here $>$

\section{Transitional Paths}

The computed transitional paths for consumption and investment depicted in Figures 1(a)1(b) change dramatically in a habit-forming model. While the real exchange rate depreciation $(\dot{p}(0)>0)$ raises the consumption-based real rate of interest, the rise in the time preference rate $\rho$ is large enough to lead to a decreasing temporal path for consumption over a first phase. The gradual fall in the reference stock reduces $\rho$ which decreases monotonically during the entire phase of adjustment. By approaching its new lower steady-state value from above, the adjustment of real consumption releases resources for capital accumulation. After $t \simeq 9$ periods, the capital stock reaches its lowest value. Investment is crowded-in since output $Y$ now exceeds the demand for the domestic good. Because the rate of return on domestic assets starts its decrease, the rate of real exchange rate depreciation gets smaller.

After 11 periods, consumption equalizes its new lower steady-state level and keeps on decreasing. At time $t \simeq 20$ periods, the declining time preference rate equalizes the slippery slope side of the consumption-based real interest rate's hump-shaped transitional path. At this point, real consumption reaches its lowest level and starts increasing. While savings rises, Figure 1(b) indicates that the current account stays always in deficit due to a positive investment flow.

\subsection{Habit Persistence and Crowding-Out of Investment}

$<$ Please insert Figures 2(a), 2(b) about here $>$

We conducted a sensitivity analysis by plotting the size of the fall in the investment rate after a rise in $g^{D}$ by 1 percentage point of GDP against the weight of habits in utility $\gamma$ and 
the speed of habits $\sigma$, allowing for these two parameters to vary from 0 to 0.95 and 0.3 to 2 respectively. These two parameters jointly determine the strength of consumption inertia in the short-term. Figures 2(a) and 2(b) show that investment is crowded-out further by public spending as habit persistence gets stronger. More specifically, as $\gamma$ increases from 0.1 to 0.9 , the rate of investment declines from $0.04 \%$ to $0.35 \%$ of initial GDP. If $\gamma$ is kept fixed to 0.8 , the corresponding decline is from $0.3 \%$ to $0.4 \%$ of initial GDP as $\sigma$ increases from 0.5 to 1.5 . The explanation is that as $\gamma$ gets closer to unity and $\sigma$ gets larger, the short-run MPC gets smaller. Hence, private demand falls by a lower amount. Consequently, investment must be crowded-out further in the short-term to eliminate the greater excess of demand in the home goods market.

\section{Conclusion}

For countries of the euro area or a country that is in a "liquidity trap", fiscal policy may be the sole instrument of stabilization policy that the government may employ. Surprisingly, however, the exploration of the macroeconomic effects of fiscal policy has only recently received notable attention in the empirical literature. In this regard, one of the empirical facts for which the profession seems to be in agreement is that a rise in public spending has a negative impact on investment. However, in seeking a theoretical justification for this apparent stylized fact, recent conclusions by Mountford and Uhlig [2005] cast doubt over standard Keynesian explanation as a possibility. Similarly, the standard open economy version of the neoclassical RBC model predicts that investment would be crowded-in by public spending. In this paper, we show that by introducing habit-forming behavior into a dynamic general equilibrium model, one can broaden the predictive power of the standard neoclassical model in order to accommodate the observed negative relationship between investment and public spending. More precisely, habit-forming consumers, confronted with a fall in their disposable income, are unwilling to cut their real expenditure by a large amount and thus prefer to reallocate their real consumption towards the present. This consumption inertia then implies that investment is crowded-out 
by fiscal spending. The reason behind this is that because consumption reacts weakly, the decline in private demand fails to offset the rise in public spending, which in turn causes a fall in investment. As long as consumers care about habits in deriving utility, the drop in savings always more than offsets the decrease in investment such that the open economy experiences a short-term current account deficit.

We also shed light on the role of habit persistence in consumption in determining the size of the crowding-out of investment via numerical experiments. These show that as habit persistence gets stronger, the crowding-out effect of fiscal expansion is smaller for real consumption and larger for investment. For example, in the case of the latter, the drop in the investment rate falls from $0.04 \%$ to around $0.35 \%$ percentage points of GDP as the weight assigned to habits in utility increases from $10 \%$ to $90 \%$. Moreover, as consumption inertia rises, the fall in savings gets larger as individuals wish to sustain their original standard of living, and thereby the open economy experiences a greater current account deficit. Finally, as trade openness increases, both investment and consumption are crowded-out by a smaller amount and exports by a larger one which worsens further the net foreign asset position.

\section{Notes}

${ }^{1}$ If agents anticipate a steady-state fall in consumption, the intertemporal marginal rate of substitution gets larger which provides an incentive for agents to reallocate consumption towards the present. Because the slope of the indifference curves along a constant consumption path rises, individuals' impatience increases. Therefore, real consumption deviates from the usual perfectly smooth temporal profile and declines over time, whenever the real interest rate is kept fixed. By shifting the time paths for consumption and thereby savings, the introduction of habits affects the long-term response of consumption to a change of private wealth dictated by the long-run IES denoted by $\nu$.

${ }^{2}$ Since labor supply is inelastic and normalized to unity, employment will be dropped from the production function.

${ }^{3}$ Since a rise in the relative price of foreign goods makes domestic goods cheaper, a real exchange rate depreciation raises exports, i. e. , $X_{p}()>$.0 .

${ }^{4}$ Linearizing the accumulation equation for habits (4), the dynamic equation for consumption (8), the accu- 
mulation equation for physical capital (9) (combined with (6)) and the dynamic equation for the real exchange rate $(7 \mathrm{~d})$, we obtain a four by four linearized system. Since the number of predetermined variables $(s$ and $k)$ equals the number of negative eigenvalues, and the number of jump variables $(c$ and $p$ ) equals the number of positive eigenvalues, there exists a unique two-dimensional convergent path towards the steady-state.

${ }^{5}$ For all parametrization, we find that $\Phi_{1}<0$ and $\Phi_{2}>0$. Accordingly, a rise in $\tilde{k}$ or a decrease in $\tilde{s}$ leads to a decumulation of traded bonds holding.

${ }^{6}$ Decreasing $\varphi$ from 0.9 to 0.5 implies a rise in the import content of consumption expenditure from $15 \%$ to $55 \%$ and an increase in the exports-GDP ratio from $15 \%$ to $36 \%$.

${ }^{7}$ Because consumption weakly reacts to the reduction of wealth when the expansionary budget policy is implemented, the savings rate falls dramatically. As $\gamma$ gets closer to 1 and $\sigma$ gets larger, the short-term MPC shrinks further. Hence, households are more reluctant to cut their real expenditure initially, and thus are more willing to reallocate their consumption towards present by decumulating further the stock of financial wealth.

\section{References}

Afonso, António, and Ricardo Sousa (2009) The Macroeconomic Effects of Fiscal Policy. European Central Bank Working Paper Series n’ 991.

Baxter, Marianne and Robert G. King (1993) Fiscal Policy in General Equilibrium. American Economic Review, 83(3), 315-334.

Blanchard, Olivier J., and Roberto Perotti (2002) An Empirical Characterization of the Dynamic Effects of Changes in Government Spending and Taxes on Output. Quarterly Journal of Economics $177,1329-1368$.

Carroll, Christopher D., Jody Overland, and David N. Weil (2000) Saving and Growth with Habit Formation. American Economic Review 90(3), 341-355.

Chinn, Menzie and Eswar S. Prasad (2003) Medium-Term Determinants of Current Accounts in Industrial and Developing Countries: An Empirical Exploration. Journal of International Economics $59,47-76$.

Corsetti, Giancarlo, and Gernot Müller (2006) Twin Deficits: Squaring Theory, Evidence and Common Sense. Economic Policy 48, 597-638.

Freund, Caroline (2005) Current Account Adjustment in Industrial Countries. Journal of International Money and Finance 24, 1278-1298. 
Karayalcin, Cem (1999) Temporary and Permanent Government Spending in a Small Open Economy Model. Journal of Monetary Economics 43, 125-141.

Mountford, Andrew and Harald Uhlig (2005) What are the Effects of Fiscal Policy Shocks? SFB 649 Discussion Papers, Humboldt University.

Perotti, Roberto (2005) Estimating the Effects of the Fiscal Policy in OECD Countries. CEPR Discussion Paper 4842.

Sommer, Martin (2007) Habit Formation and Aggregate Consumption Dynamics. The B.E. Journal of Macroeconomics, 7(1) (Advances), Article 21. 


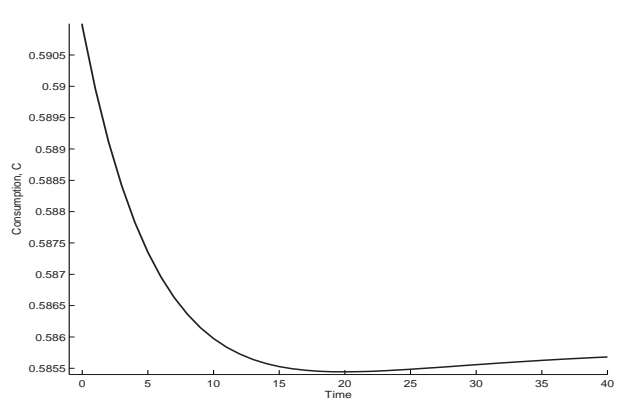

(a) Consumption

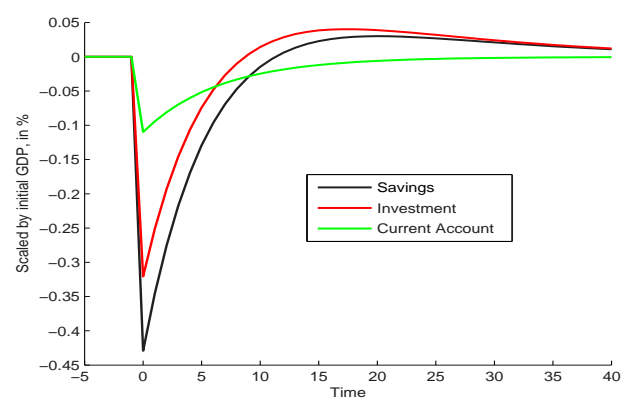

(b) Savings, Invest., Current Account

Figure 1: Computed Transitional Paths

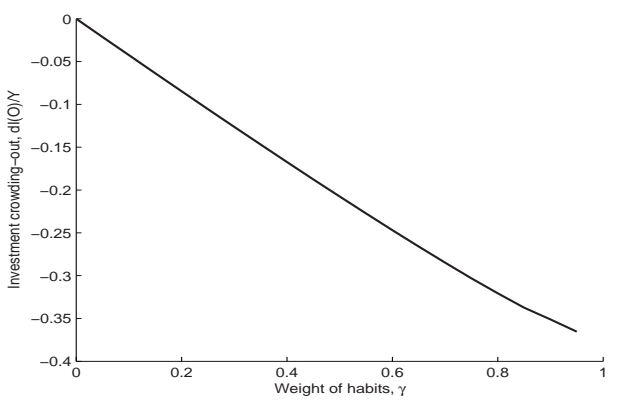

(a) Plot of $\mathrm{d} I(0) / Y$ against $\gamma$

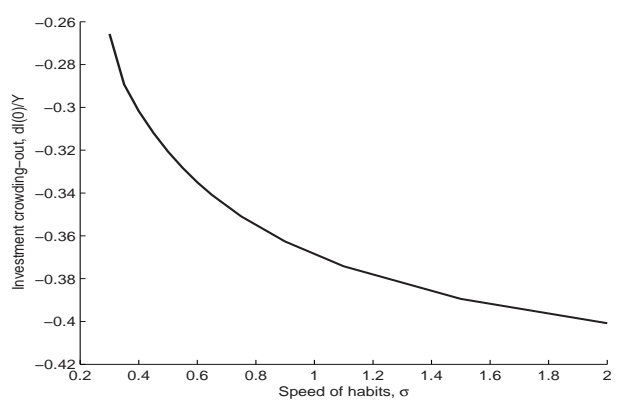

(b) Plot of $\mathrm{d} I(0) / Y$ against $\sigma$

Figure 2: Sensitivity of the Crowding-Out Effect to $\gamma$ and $\sigma$

Table 1: Quantitative Effects of a Permanent Fiscal Expansion (in \%)

\begin{tabular}{|r|ccccc|}
\hline Variables $^{a}$ & TS Pref. $\gamma=0$ & $(\gamma=0.8, \sigma=0.5)$ & $(\gamma=0.3)$ & $(\sigma=0.9)$ & $(\varphi=0.5)$ \\
\hline A.Long-Term & & & & & \\
Consumption $p_{c} \tilde{c}$ & -0.80 & -0.85 & -0.81 & -083 & -0.48 \\
Cons. in home good $\tilde{c}^{D} \tilde{\text { Exports } \tilde{X}}$ & -0.84 & -0.86 & -0.84 & -0.85 & -0.55 \\
B.Short-Term & -0.16 & -0.14 & -0.16 & -0.15 & -0.45 \\
Consumption $p_{c} c(0)$ & -0.80 & -0.34 & -0.63 & -0.31 & -0.17 \\
Cons. in home good $c^{D}(0)$ & -0.84 & -0.49 & -0.71 & -0.46 & -0.44 \\
Exports $X(0)$ & -0.16 & -0.19 & -0.16 & -0.18 & -0.48 \\
Investment rate $I(0)$ & 0.00 & -0.32 & -0.13 & -0.36 & -0.09 \\
Savings rate $S(0)$ & 0.00 & -0.43 & -0.16 & -0.47 & -0.23 \\
Current account $c a(0)$ & 0.00 & -0.11 & -0.04 & -0.11 & -0.15 \\
\hline
\end{tabular}

${ }^{a}$ We consider an increase in $g^{D}$ by 1 percentage point of GDP. All effects are scaled by initial GDP. TS Pref.: time separable preferences. 


\section{A Short-Run Static Solution}

Solving the domestic goods market-clearing condition (9) for the rate of investment in capital goods:

$$
I=I\left(\bar{\lambda}, c, p, k, g^{D}\right)
$$

where partial derivatives are given by

$$
\begin{aligned}
I_{c} & =\frac{\partial I}{\partial c}=-\left(1-\alpha_{c}\right) p_{c}<0 \\
I_{p} & =\frac{\partial I}{\partial p}=-\left(X_{p}-p p_{c}^{\prime \prime} c\right)<0 \\
I_{k} & =\frac{\partial I}{\partial k}=F_{k}>0 \\
I_{g^{D}} & =\frac{\partial I}{\partial g_{d}}=-1<0 .
\end{aligned}
$$

\section{B Equilibrium Dynamics and Formal Solutions}

In this section, making use of general forms for preferences and production function, we prove that the dynamic system exhibits saddle-point stability.

$$
(\dot{s}, \dot{c}, \dot{k}, \dot{p})^{T}=J(s(t)-\tilde{s}, c(t)-\tilde{c}, k(t)-\tilde{k}, p(t)-\tilde{p})^{T},
$$

where $J$ is given by

$$
J \equiv\left(\begin{array}{cccc}
-\sigma & \sigma & 0 & 0 \\
a_{21} & \delta+\sigma & a_{23} & a_{24} \\
0 & I_{c} & r^{\star} & I_{p} \\
0 & 0 & \tilde{p} F_{k k} & 0
\end{array}\right)
$$

where we used the fact that $I_{k}-\delta_{K}=r^{\star}$ we let

$$
\Gamma=u_{c s}+\frac{\sigma}{\delta+2 \sigma} u_{s s}>0 .
$$


and

$$
\begin{aligned}
a_{21} & =\frac{\delta+2 \sigma}{u_{c c}} \Gamma<0, \\
a_{23} & =-\frac{\bar{\lambda} p_{c}}{\tilde{p} u_{c c}}\left(1-\alpha_{c}\right) F_{k k}>0, \\
a_{24} & =\frac{\bar{\lambda} p_{c}(\delta+\sigma)}{u_{c c} \tilde{p}^{2}}\left(1-\alpha_{c}\right)<0 .
\end{aligned}
$$

The sign of $\Gamma$ depends on $u_{c s}$. If the marginal utility of real consumption is sufficiently increasing in stock of habits, the preferences of the representative consumer display adjacent complementarity and $\Gamma$ is positive (see Ryder and Heal [1973]). If $u_{c s}$ has a negative or a small positive value, $\Gamma$ is negative and preferences are said to display distant complementarity. We assume that $\Gamma$ is positive which holds for the utility function (??) as longer as $\gamma>0$.

\section{Saddle-Point Stability}

Denoting by $\mu$ the eigenvalue, the characteristic equation for the matrix (15) of the linearized system writes as follows:

$$
\mu^{4}+b_{1} \mu^{3}+b_{2} \mu^{2}+b_{3} \mu+b_{4}=0
$$

with

$$
\begin{aligned}
& b_{1}=-\operatorname{tr} J=-2 r^{\star}<0 \\
& b_{2}=M_{2}=\left(r^{\star}\right)^{2}-\left\{\frac{\sigma(\delta+\sigma)}{u_{c c}}\left[u_{c c}+\left(\frac{\delta+2 \sigma}{\delta+\sigma}\right) \Gamma\right]+\tilde{p} F_{k k}\left[I_{p}-I_{c} \frac{\bar{\lambda} p_{c}}{u_{c c} \tilde{p}^{2}}\left(1-\alpha_{c}\right)\right]\right\} \gtrless 0,(1 \\
& b_{3}=-M_{3}=r^{\star}\left\{\frac{\sigma(\delta+\sigma)}{u_{c c}}\left[u_{c c}+\left(\frac{\delta+2 \sigma}{\delta+\sigma}\right) \Gamma\right]+\tilde{p} F_{k k}\left[I_{p}-I_{c} \frac{\bar{\lambda} p_{c}}{u_{c c} \tilde{p}^{2}}\left(1-\alpha_{c}\right)\right]\right\}>0 \\
& b_{4}=\operatorname{Det} J=\frac{\sigma(\delta+\sigma) \tilde{p} F_{k k}}{u_{c c}}\left\{I_{p}\left[u_{c c}+\left(\frac{\delta+2 \sigma}{\delta+\sigma}\right) \Gamma\right]-I_{c} \frac{\bar{\lambda} p_{c}}{\tilde{p}^{2}}\left(1-\alpha_{c}\right)\right\}>0
\end{aligned}
$$

where $J$ is given by (15) and $M_{2}$ and $M_{3}$ are respectively the sum of all diagonal second and third order minors of $J$ (see Dockner and Feichtinger [1991], p. 45). The positive signs of (19c) and (19d) stem from the following (imposed) inequality

$$
u_{c s}+\frac{\sigma}{\delta+2 \sigma} u_{s s}<-\left(\frac{\delta+\sigma}{\delta+2 \sigma}\right) u_{c c}
$$

Imposing (20) ensures that adjacent complementarity is not too strong and that the dynamic system exhibits a saddle point stability (see Becker and Murphy [1988]). 
The characteristic polynomial of degree four (18) can be rewritten as a characteristic polynomial of second degree

$$
\theta^{2}+\frac{b_{3}}{r^{\star}} \theta+b_{4} \quad \text { with } \quad \theta=\mu_{i}\left(r^{\star}-\mu_{i}\right) .
$$

By evaluating first the eigenvalues $\theta$ from the second order polynomial and then calculating $\mu$ from the definition of $\theta$, the four eigenvalues of the upper-left four by four sub-matrix in the Jacobian are given by:

$$
\mu_{i} \equiv \frac{1}{2}\left\{r^{\star} \pm \sqrt{\left(r^{\star}\right)^{2}+2\left(\frac{b_{3}}{r^{\star}} \pm \sqrt{\left(\frac{b_{3}}{r^{\star}}\right)^{2}-4 b_{4}}\right)}\right\}, \quad i=1,2,3,4
$$

with

$$
\mu_{1}<\mu_{2}<0<r^{\star}<\mu_{3}<\mu_{4}
$$

and having the following properties

$$
r^{\star}-\mu_{1}=\mu_{4}, \quad r^{\star}-\mu_{2}=\mu_{3} .
$$

The determinant of matrix $J$ given by (19d) is positive, i. e. $a_{4}>0$. This is consistent with there being either 2 negative and 2 positive roots, 4 positive, or 4 negative roots. Since the trace of the matrix $J$ is equal to $2 r^{\star}=-a_{1}$ (which is positive) and the trace is equal to the sum of eigenvalues, only the first two cases have to be considered. By Descartes rule of signs, necessary and sufficient conditions for the characteristic equation to have just two positive roots is that either $a_{2}<0$ or $a_{3}>0$. From (19c), $a_{3}>0$ so we can exclude the case of four positive roots. Since the system features two state variables, $s$ and $k$, and two jump variables, $c$ and $p$, the equilibrium yields a unique stable saddle-path.

Following Dockner and Feichtinger [1991], the necessary and sufficient conditions for saddlepoint stability with real roots write as follows:

$$
\begin{aligned}
& \frac{b_{3}}{r^{\star}}>0, \\
& 0<4 b_{4} \leq\left(\frac{b_{3}}{r^{\star}}\right)^{2} .
\end{aligned}
$$


The first condition (25a) holds if the inequality (20) is respected. Expression $\left(\frac{b_{3}}{r^{*}}\right)^{2}-4 b 4$ can be rewritten as:

$$
\begin{aligned}
\left(\frac{b_{3}}{r^{\star}}\right)^{2}-4 b_{4} & =\left\{\frac{\sigma(\delta+\sigma)}{u_{c c}}\left[u_{c c}+\left(\frac{\delta+2 \sigma}{\delta+\sigma}\right) \Gamma\right]-\tilde{p} F_{k k}\left[I_{p}-I_{c} \frac{\bar{\lambda} p_{c}}{u_{c c} \tilde{p}^{2}}\left(1-\alpha_{c}\right)\right]\right\}^{2} \\
& -4 \frac{\sigma(\delta+2 \sigma) F_{k k}}{u_{c c}} \Gamma I_{c} \frac{\bar{\lambda} p_{c}}{u_{c c} \tilde{p}}\left(1-\alpha_{c}\right) \gtreqless 0 .
\end{aligned}
$$

As the second condition (25b) may quite plausibly not hold, the system (14) exhibits saddlepoint behavior (as we have shown previously) but the stable roots may be either real or complex. In the former case, trajectories may be monotonic or humped. In the latter case, the dynamics involve cyclical behavior. As we shall see later, for plausible values of $\sigma$, formal solution are real-valued.

\section{Formal Solutions}

Setting the constants $A_{3}=A_{4}=0$ to insure a converging adjustment for all macroeconomic aggregates, the stable paths are given by:

$$
\begin{aligned}
& s(t)-\tilde{s}=A_{1} e^{\mu_{1} t}+A_{2} e^{\mu_{2} t}, \\
& c(t)-\tilde{c}=\omega_{2}^{1} A_{1} e^{\mu_{1} t}+\omega_{2}^{2} A_{2} e^{\mu_{2} t}, \\
& k(t)-\tilde{k}=\omega_{3}^{1} A_{1} e^{\mu_{1} t}+\omega_{3}^{2} A_{2} e^{\mu_{2} t}, \\
& p(t)-\tilde{p}=\omega_{4}^{1} A_{1} e^{\mu_{1} t}+\omega_{4}^{2} A_{2} e^{\mu_{2} t},
\end{aligned}
$$

where the eigenvectors $\omega_{j}^{i}$ associated with eigenvalue $\mu_{i}$ are given by

$$
\begin{aligned}
\omega_{2}^{i} & =\left(\frac{\sigma+\mu_{i}}{\sigma}\right) \\
\omega_{3}^{i} & =-\frac{I_{c}\left(\sigma+\mu_{i}\right) \mu_{i}}{\sigma\left[\left(r^{\star}-\mu_{i}\right) \mu_{i}+I_{p} \tilde{p} F_{k k}\right]} \\
\omega_{4}^{i} & =\frac{\tilde{p} F_{k k}}{\mu_{i}} \omega_{3}^{i}=-\tilde{p} F_{k k} \frac{I_{c}\left(\sigma+\mu_{i}\right)}{\sigma\left[\left(r^{\star}-\mu_{i}\right) \mu_{i}+I_{p} \tilde{p} F_{k k}\right]} .
\end{aligned}
$$

We normalized $\omega_{1}^{i}$ to unity. The two constants write as follows

$$
A_{1}=\frac{\mathrm{d} \tilde{k}-\omega_{3}^{2} \mathrm{~d} \tilde{s}}{\omega_{3}^{2}-\omega_{3}^{1}}, \quad A_{2}=\frac{\omega_{3}^{1} \mathrm{~d} \tilde{s}-\mathrm{d} \tilde{k}}{\omega_{3}^{2}-\omega_{3}^{1}} .
$$

To determine the signs of eigenvectors, we have to establish the signs of useful expressions.

The first one is straightforward:

$$
\frac{a_{3}}{r^{\star}}>\sqrt{\left(\frac{a_{3}}{r^{\star}}\right)^{2}-4 a_{4}}>0
$$


Making use of (22), we can establish some conditions for the signs of the following expressions:

$$
\begin{array}{lll}
\mu_{1} \mu_{4}+I_{p} \tilde{p} F_{k k} \lessgtr 0 & \text { depending on wether } & \tilde{p} F_{k k} I_{p} \lessgtr \sigma(\delta+\sigma), \\
\mu_{2} \mu_{3}+I_{p} \tilde{p} F_{k k} \gtrless 0 & \text { depending on wether } & \tilde{p} F_{k k} I_{p} \gtrless \sigma(\delta+\sigma) .
\end{array}
$$

Since for plausible (and a large range of) values of preferences and production-side parameters, inequality $0<\tilde{p} F_{k k} I_{p}<\sigma(\delta+\sigma)$ numerically holds, we deduce that $\mu_{1} \mu_{4}+I_{p} \tilde{p} F_{k k}<0$ and $\mu_{2} \mu_{3}+I_{p} \tilde{p} F_{k k}<0$ (see inequality (32b)).

From the discussion above, we assume thereafter that $0<\tilde{p} F_{k k} I_{p}<\sigma(\delta+\sigma)$ which implies in turn:

$$
\begin{aligned}
\left(r^{\star}-\mu_{1}\right) \mu_{1}+I_{p} \tilde{p} F_{k k} & =\mu_{4} \mu_{1}+I_{p} \tilde{p} F_{k k}<0, \\
\left(r^{\star}-\mu_{2}\right) \mu_{2}+I_{p} \tilde{p} F_{k k} & =\mu_{3} \mu_{2}+I_{p} \tilde{p} F_{k k}<0 .
\end{aligned}
$$

In addition, the sign of eigenvector $\left(\sigma+\mu_{1}\right)$ can be established as follows:

$$
\left(\sigma+\mu_{1}\right) \gtrless 0 \text { depending on wether } \quad \tilde{p} F_{k k} I_{p} \lessgtr \sigma(\delta+\sigma) \text {. }
$$

Imposing the inequality has the following implications for eigenvectors $\omega_{2}^{1}$ and $\omega_{2}^{2}$ :

$$
\left(\sigma+\mu_{1}\right)>0, \quad\left(\sigma+\mu_{2}\right)>0 \quad \text { if } \quad \tilde{p} F_{k k} I_{p}<\sigma(\delta+\sigma)
$$

where it is straightforward to deduce that $\left(\sigma+\mu_{2}\right)>0$ since $\mu_{1}<\mu_{2}<0$.

\section{Signs of Eigenvectors}

We write out the four eigenvectors $\omega^{i}$, corresponding with stable eigenvalues $\mu_{i}$ with $i=1,2$, to determine their signs:

$$
\omega^{1}=\left(\begin{array}{cc}
1 & (+) \\
\left(\frac{\sigma+\mu_{1}}{\sigma}\right) & (+) \\
-\frac{I_{c}\left(\sigma+\mu_{1}\right) \mu_{1}}{\sigma\left[\left(r^{\star}-\mu_{1}\right) \mu_{1}+I_{p} \tilde{p} F_{k k}\right]} & (+) \\
-\tilde{p} F_{k k} \frac{I_{c}\left(\sigma+\mu_{1}\right)}{\sigma\left[\left(r^{\star}-\mu_{1}\right) \mu_{1}+I_{p} \tilde{p} F_{k k}\right]} & (+)
\end{array}\right), \quad \omega^{2}=\left(\begin{array}{cc}
1 & (+) \\
\left(\frac{\sigma+\mu_{2}}{\sigma}\right) & (+) \\
-\frac{I_{c}\left(\sigma+\mu_{2}\right) \mu_{2}}{\sigma\left[\left(r^{\star}-\mu_{2}\right) \mu_{2}+I_{p} \tilde{p} F_{k k}\right]} & (+) \\
-\tilde{p} F_{k k} \frac{I_{c}\left(\sigma+\mu_{2}\right)}{\sigma\left[\left(r^{\star}-\mu_{2}\right) \mu_{2}+I_{p} \tilde{p} F_{k k}\right]} & (+)
\end{array}\right) .
$$




\section{Formal Solution for the Stock of Foreign Assets}

Using the market clearing condition (9), we may express the current account equation given by (5) in terms of net exports:

$$
\dot{b}=r^{\star} b+\frac{X(p)}{p}-p_{c}^{\prime}(p) c-g^{F}
$$

where the term $X / p-c^{F}-g^{F}$ represents the balance of trade measured in terms of the foreign good.

We first linearize equation (36) around the steady-state:

$$
\dot{b}(t)=r^{\star}(b(t)-\tilde{b})+\tilde{\Omega}(p(t)-\tilde{p})-p_{c}^{\prime}(\tilde{p})(c(t)-\tilde{c})
$$

with

$$
\tilde{\Omega} \equiv \frac{1}{\tilde{p}}\left[\frac{\tilde{X}}{\tilde{p}}\left(\tilde{\nu}_{X}-1\right)+\tilde{c}^{F} \tilde{\nu}_{F}\right]=-\frac{1}{\tilde{p}}\left[I_{p}+\frac{\tilde{X}}{\tilde{p}}\right]>0,
$$

where $\nu_{X} \equiv \frac{X_{p} p}{X}>0$ and $\nu_{F}=-\frac{p_{c}^{\prime \prime} p}{p}=\phi\left(1-\alpha_{c}\right)>0$ represent respectively the elasticities of domestic exports and imports. Expression (38) gives the net exports reaction expressed in terms of the foreign good to a change in the real exchange rate. We assume that the generalized version of the Marshall-Lerner condition, that is with an unbalanced trade balance, holds. Hence, a rise in the relative price of import goods leads to an improvement of the trade balance evaluated at the steady-state, so we set $\tilde{\Omega}>0$. Second equality of (38) has been obtained by substituting the expression of $I_{p}$ which is equal to $-\left(X_{p}-p p_{c}^{\prime \prime} c\right)$.

Inserting the stable solutions for $(p(t)-\tilde{p})$ and $(c(t)-\tilde{c})$ given respectively by $(27 \mathrm{~d})$ and (27b), the solution for the current account writes as follows :

$$
\dot{b}(t)=r^{\star}(b(t)-\tilde{b})+\tilde{\Omega} \sum_{i=1}^{2} A_{i} \omega_{4}^{i} e^{\mu_{i} t}-p_{c}^{\prime} \sum_{i=1}^{2} A_{i} \omega_{2}^{i} e^{\mu_{i} t} .
$$

Solving the differential equation leads to:

$$
\begin{aligned}
b(t)-\tilde{b} & =\left[\left(b_{0}-\tilde{b}\right)-\frac{N_{1} A_{1}}{\mu_{1}-r^{\star}}-\frac{N_{2} A_{2}}{\mu_{2}-r^{\star}}\right] e^{r^{\star} t} \\
& +\frac{N_{1} A_{1}}{\mu_{1}-r^{\star}} e^{\mu_{1} t}+\frac{N_{2} A_{2}}{\mu_{2}-r^{\star}} e^{\mu_{2} t}
\end{aligned}
$$

where

$$
N_{1}=\tilde{\Omega} \omega_{4}^{1}-p_{c}^{\prime}\left(\frac{\sigma+\mu_{1}}{\sigma}\right), \quad N_{2}=\tilde{\Omega} \omega_{4}^{2}-p_{c}^{\prime}\left(\frac{\sigma+\mu_{2}}{\sigma}\right)
$$


Invoking the transversality condition for intertemporal solvency, i. e. equation (??), we obtain the linearized version of the nation's intertemporal budget constraint:

$$
b_{0}-\tilde{b}=\frac{N_{1} A_{1}}{\mu_{1}-r^{\star}}+\frac{N_{2} A_{2}}{\mu_{2}-r^{\star}} .
$$

For the national intertemporal solvency to hold, the terms in brackets of equation (40) must be null, so the stable solution for net foreign assets finally reduces to

$$
b(t)-\tilde{b}=\frac{N_{1} A_{1}}{\mu_{1}-r^{\star}} e^{\mu_{1} t}+\frac{N_{2} A_{2}}{\mu_{2}-r^{\star}} e^{\mu_{2} t} .
$$

Inserting the values for the constants $A_{1}$ and $A_{2}$ given by equations (37), we obtain (after some tedious computations) the linearized version of the national intertemporal budget constraint expressed as a function of initial stocks of capital and habits:

$$
\tilde{b}-b_{0}=\Phi_{1}\left(\tilde{k}-k_{0}\right)+\Phi_{2}\left(\tilde{s}-s_{0}\right)
$$

with

$$
\begin{aligned}
\Phi_{1} & =\frac{\left(\mu_{1}-r^{\star}\right) N_{2}-\left(\mu_{2}-r^{\star}\right) N_{1}}{\left(\mu_{1}-r^{\star}\right)\left(\mu_{2}-r^{\star}\right)\left(\omega_{3}^{2}-\omega_{3}^{1}\right)} \lessgtr 0, \\
\Phi_{2} & =\frac{\left(\mu_{2}-r^{\star}\right) \omega_{3}^{2} N_{1}-\left(\mu_{1}-r^{\star}\right) \omega_{3}^{1} N_{2}}{\left(\mu_{1}-r^{\star}\right)\left(\mu_{2}-r^{\star}\right)\left(\omega_{3}^{2}-\omega_{3}^{1}\right)}>0,
\end{aligned}
$$

where $\left(\omega_{3}^{2}-\omega_{3}^{1}\right)>0$ is given by

$$
\omega_{3}^{2}-\omega_{3}^{1}=\frac{I_{c}\left(\mu_{1}-\mu_{2}\right)\left\{\mu_{1} \mu_{2}\left(\sigma+r^{\star}\right)+I_{p} \tilde{p} F_{k k}\left[\sigma+\left(\mu_{1}+\mu_{2}\right)\right]\right\}}{\sigma\left[\mu_{1} \mu_{4}+I_{p} \tilde{p} F_{k k}\right]\left[\mu_{2} \mu_{3}+I_{p} \tilde{p} F_{k k}\right]}=\frac{(+)}{(+)}>0,
$$

where we have used the fact that $\mu_{3}-\mu_{4}=\mu_{1}-\mu_{2}$ and $\mu_{1} \mu_{3}-\mu_{2} \mu_{4}=r^{\star}\left(\mu_{1}-\mu_{2}\right)$. The sign of (46) stems from: $\mu_{1}-\mu_{2}<0, I_{c}<0$, together with

$$
\begin{aligned}
& \mu_{1} \mu_{2}\left(\sigma+r^{\star}\right)+I_{p} \tilde{p} F_{k k}\left[\sigma+\left(\mu_{1}+\mu_{2}\right)\right] \\
= & {\left[I_{p} \tilde{p} F_{k k}+\mu_{1} \mu_{2}\right]\left(\sigma+\mu_{1}\right)+\mu_{2}\left[I_{p} \tilde{p} F_{k k}+\mu_{1} \mu_{4}\right]=(+)+(+)>0, }
\end{aligned}
$$

where $\left[I_{p} \tilde{p} F_{k k}+\mu_{1} \mu_{4}\right]<0($ see $(32 \mathrm{a}))$.

In addition, we have computed the following expressions:

$$
\begin{aligned}
& \left(\mu_{2}-r^{\star}\right) \omega_{3}^{2} N_{1}-\left(\mu_{1}-r^{\star}\right) \omega_{3}^{1} N_{2} \\
= & \frac{\left(1-\alpha_{c}\right)\left(p_{c}\right)^{2} F_{k k}\left(I_{p}+\left(1-\alpha_{c}\right) \frac{\tilde{X}}{\tilde{p}}\right)}{\sigma^{2}\left[\left(r^{\star}-\mu_{1}\right) \mu_{1}+I_{p} \tilde{p} F_{k k}\right]\left[\left(r^{\star}-\mu_{2}\right) \mu_{2}+I_{p} \tilde{p} F_{k k}\right]} \\
\times & \frac{\left(\sigma+\mu_{1}\right)\left(\sigma+\mu_{2}\right)\left(\mu_{2}-\mu_{1}\right)\left[r^{\star}-\left(\mu_{1}+\mu_{2}\right)\right]}{\sigma^{2}\left[\left(r^{\star}-\mu_{1}\right) \mu_{1}+I_{p} \tilde{p} F_{k k}\right]\left[\left(r^{\star}-\mu_{2}\right) \mu_{2}+I_{p} \tilde{p} F_{k k}\right]}=\frac{(+)}{(+)}>0 .
\end{aligned}
$$


and

$$
\begin{aligned}
& \left(\mu_{1}-r^{\star}\right) N_{2}-\left(\mu_{2}-r^{\star}\right) N_{1} \\
= & p_{c} \tilde{p} F_{k k}\left[I_{p}+\left(1-\alpha_{c}\right) \frac{\tilde{X}}{\tilde{p}}\right] \frac{\left[I_{p} \tilde{p} F_{k k}\left(\mu_{2}-\mu_{1}\right)\left(\sigma+r^{\star}\right)+\left(\sigma+\mu_{2}\right) \mu_{1}\left(\mu_{4}\right)^{2}-\left(\sigma+\mu_{1}\right) \mu_{2}\left(\mu_{3}\right)^{2}\right]}{\tilde{p} \sigma\left[\left(r^{\star}-\mu_{1}\right) \mu_{1}+I_{p} \tilde{p} F_{k k}\right]\left[\left(r^{\star}-\mu_{2}\right) \mu_{2}+I_{p} \tilde{p} F_{k k}\right]} \\
& +\alpha_{c} p_{c} \frac{\mu_{3} \mu_{4}\left(\mu_{2}-\mu_{1}\right)\left[I_{p} \tilde{p} F_{k k}\left[\sigma+\left(\mu_{1}+\mu_{2}\right)\right]+\mu_{1} \mu_{2}\left(\sigma+r^{\star}\right)\right]}{\tilde{p} \sigma\left[\left(r^{\star}-\mu_{1}\right) \mu_{1}+I_{p} \tilde{p} F_{k k}\right]\left[\left(r^{\star}-\mu_{2}\right) \mu_{2}+I_{p} \tilde{p} F_{k k}\right]} \gtrless 0 .
\end{aligned}
$$

By using the second equality of (38) and after some algebra, $N_{1}$ and $N_{2}$ can be rewritten as follows

$$
\begin{aligned}
N_{1} & =-p_{c}\left(\frac{\sigma+\mu_{1}}{\sigma}\right)\left\{\frac{\tilde{p} F_{k k}\left(I_{p}+\left(1-\alpha_{c}\right) \frac{\tilde{X}}{\tilde{p}}\right)+\alpha_{c} \mu_{4} \mu_{1}}{\tilde{p}\left[\mu_{4} \mu_{1}+I_{p} \tilde{p} F_{k k}\right]}\right\} \lessgtr 0, \\
& =(-) \times\left\{\frac{(+)+(-)}{(-)}\right\} . \\
N_{2} & =-p_{c}\left(\frac{\sigma+\mu_{2}}{\sigma}\right)\left\{\frac{\tilde{p} F_{k k}\left(I_{p}+\left(1-\alpha_{c}\right) \frac{\tilde{X}}{\tilde{p}}\right)+\alpha_{c} \mu_{3} \mu_{2}}{\tilde{p}\left[\mu_{3} \mu_{2}+I_{p} \tilde{p} F_{k k}\right]}\right\} \gtrless 0, \\
& =(-) \times\left\{\frac{(+)+(-)}{(-)}\right\} .
\end{aligned}
$$

The signs of $N_{1}$ and $N_{2}$ remain undetermined since the numerator is the sum of a positive term and a negative term. We do not impose any sign to expressions (50a)-(50a) but for all parametrization, simulations indicate that $N_{1}<0$ whereas $N_{2} \gtrless 0$. The sign of $N_{2}$ relies upon the strength of habit persistence in consumption which size depends on $\gamma$ and $\sigma$; furthermore, its sign is quite sensitive to the intratemporal elasticity of substitution $\phi$ between $c^{D}$ and $c^{F}$ since this parameter influences the magnitude of the first in square brackets (i. e. $I_{p}$ ), that is the Marshall-Lerner condition.

We have computed an expression of $\Phi_{1}$ by making use of (49) and (46) in order to have an analytical expression that allows to bring out the parameters which influences its size:

$$
\begin{aligned}
\Phi_{1}= & -\frac{F_{k k}\left[I_{p}+\left(1-\alpha_{c}\right) \frac{\tilde{X}}{\tilde{p}}\right]\left[I_{p} \tilde{p} F_{k k}\left(\mu_{2}-\mu_{1}\right)\left(\sigma+r^{\star}\right)+\left(\sigma+\mu_{2}\right) \mu_{1}\left(\mu_{4}\right)^{2}-\left(\sigma+\mu_{1}\right) \mu_{2}\left(\mu_{3}\right)^{2}\right]}{\left(1-\alpha_{c}\right) \mu_{3} \mu_{4}\left(\mu_{1}-\mu_{2}\right)\left\{\mu_{1} \mu_{2}\left(\sigma+r^{\star}\right)+I_{p} \tilde{p} F_{k k}\left[\sigma+\left(\mu_{1}+\mu_{2}\right)\right]\right\}} \\
& +\frac{1}{\tilde{p}}\left(\frac{\alpha_{c}}{1-\alpha_{c}}\right) \lessgtr 0 .
\end{aligned}
$$

In addition, we have evaluated $\Phi_{2}$ by making use of (48) and (46):

$$
\Phi_{2}=\frac{p_{c} F_{k k}\left[I_{p}+\left(1-\alpha_{c}\right) \frac{\tilde{X}}{\tilde{p}}\right]\left(\sigma+\mu_{1}\right)\left(\sigma+\mu_{2}\right)\left[r^{\star}-\left(\mu_{1}+\mu_{2}\right)\right]}{\sigma \mu_{3} \mu_{4}\left\{\mu_{1} \mu_{2}\left(\sigma+r^{\star}\right)+I_{p} \tilde{p} F_{k k}\left[\sigma+\left(\mu_{1}+\mu_{2}\right)\right]\right\}}=\frac{(+)}{(+)}>0,
$$


where the sign of (51) comes from the assumption of a low value of $\alpha_{c}$ as data suggest it (close to $20 \%$ ). The sign of (52) has been determined by rewriting the term in square brackets located in the denominator as follows:

$$
\begin{aligned}
& \mu_{1} \mu_{2} \sigma\left(\sigma+r^{\star}\right)+I_{p} \tilde{p} F_{k k} \sigma\left[\sigma+\left(\mu_{1}+\mu_{2}\right)\right] \\
= & -\mu_{1} \mu_{2}\left[I_{p} \tilde{p} F_{k k}+\mu_{1} \mu_{4}\right]+\left(\sigma+\mu_{1}\right)\left[\mu_{1} \mu_{2}\left(\sigma+\mu_{4}\right)+I_{p} \tilde{p} F_{k k}\left(\sigma+\mu_{2}\right)\right]>0,
\end{aligned}
$$

where we used the fact that $\sigma\left[\sigma+\left(\mu_{1}+\mu_{2}\right)\right]=\left(\sigma+\mu_{1}\right)\left(\sigma+\mu_{2}\right)-\mu_{1} \mu_{2}$ and $\sigma\left(\sigma+r^{\star}\right)=$ $\sigma\left[\sigma+\left(\mu_{1}+\mu_{4}\right)\right]=\left(\sigma+\mu_{1}\right)\left(\sigma+\mu_{4}\right)-\mu_{1} \mu_{4}$.

\section{Formal Solution for the Stock of Financial Wealth}

Financial wealth measured in terms of the domestic good, $a(t)$, is equal to the sum of the stock of foreign assets, $p(t) b(t)$, measured in terms of the foreign good and the capital stock. The law of motion for financial wealth $(S(t)=\dot{a}(t))$ is given by:

$$
\dot{a}(t)=r^{K}(t) a(t)+w(t)-p_{c}(p(t)) c-T,
$$

where lump-sump taxes cover overall government spending, i. e. $T=g^{D}+p g^{F}$. Remembering that $w=F_{n}$ and $r^{K}=F_{k}-\delta_{K}$, and linearizing (53) in the neighborhood of the steady-state, we get:

$$
\dot{a}(t)=r^{\star}(a(t)-\tilde{a})+F_{k k} \tilde{p} \tilde{b}(k(t)-\tilde{k})-p_{c}(c(t)-\tilde{c})-\left(\tilde{c}^{F}+g^{F}\right)(p(t)-\tilde{p}),
$$

where we used the fact that $\tilde{r}^{K} \equiv F_{k}-\delta_{K}=r^{\star}$ at the steady-state; we used property $F_{k n}=$ $-\frac{k}{n} F_{k k}$ of the linear homogenous function and $a \equiv p b+k$ to rewrite $F_{k k} \tilde{a}+F_{k n}$ as $F_{k k} \tilde{p} \tilde{b}$.

By inserting the stable solutions, using the fact that $\omega_{3}^{i}=\frac{\mu_{i}}{\tilde{p} F_{k k}} \omega_{4}^{i}$, and rearranging terms, the solution for the stock of financial wealth writes as follows:

$$
\dot{a}(t)=r^{\star}(a(t)-\tilde{a})-\sum_{i=1}^{2}\left[\left(r^{\star}-\mu_{i}\right) \tilde{b}+\frac{\tilde{X}}{\tilde{p}}\right] \omega_{4}^{i} A_{i} e^{\mu_{i} t}-p_{c} \sum_{i=1}^{2} A_{i} \omega_{2}^{i} e^{\mu_{i} t}
$$

where we used the fact that $\left(\tilde{c}^{F}+g^{F}\right)=r^{\star} \tilde{b}+\frac{\tilde{X}}{\tilde{p}}$.

Solving the differential equation leads to:

$$
a(t)-\tilde{a}=\left[\left(a_{0}-\tilde{a}\right)-\frac{S_{1} A_{1}}{\mu_{1}-r^{\star}}-\frac{S_{2} A_{2}}{\mu_{2}-r^{\star}}\right] e^{r^{\star} t}+\frac{S_{1} A_{1}}{\mu_{1}-r^{\star}} e^{\mu_{1} t}+\frac{S_{2} A_{2}}{\mu_{2}-r^{\star}} e^{\mu_{2} t},
$$


where

$$
\begin{aligned}
& S_{1}=-\left[\mu_{4} \tilde{b}+\frac{\tilde{X}}{\tilde{p}}\right] \omega_{4}^{1}-p_{c} \omega_{2}^{1}<0, \\
& S_{2}=-\left[\mu_{3} \tilde{b}+\frac{\tilde{X}}{\tilde{p}}\right] \omega_{4}^{2}-p_{c} \omega_{2}^{2}<0,
\end{aligned}
$$

with $\omega_{4}^{1}>, \omega_{4}^{2}>0, \omega_{2}^{1}>0, \omega_{2}^{2}>0$.

Invoking the transversality condition for intertemporal solvency, we get the linearized version of the households' intertemporal budget constraint:

$$
a_{0}-\tilde{a}=\frac{S_{1} A_{1}}{\mu_{1}-r^{\star}}+\frac{S_{2} A_{2}}{\mu_{2}-r^{\star}} .
$$

For the intertemporal solvency to hold, the terms in brackets of equation (58) must be null, so as the stable solution for the stock of financial wealth finally reduces to:

$$
a(t)-\tilde{a}=\frac{S_{1} A_{1}}{\mu_{1}-r^{\star}} e^{\mu_{1} t}+\frac{S_{2} A_{2}}{\mu_{2}-r^{\star}} e^{\mu_{2} t} .
$$

\section{Steady-State Changes of Government Spending Shocks}

\section{C.1 Steady-State Changes}

Totally differentiating equations (10) yields in matrix form

$$
\begin{aligned}
& \left(\begin{array}{ccccc}
{\left[u_{c c}+\left(\frac{\delta+2 \sigma}{\delta+\sigma}\right) \Gamma\right]} & \frac{p_{c} \bar{\lambda}\left(1-\alpha_{c}\right)}{\tilde{p}^{2}} & -\frac{p_{c}}{\tilde{p}} & 0 & 0 \\
0 & 0 & 0 & F_{k k} & 0 \\
-p_{c} & -\left(\tilde{c}^{F}+g^{F}-r^{\star} \tilde{b}\right) & 0 & r^{\star} & \tilde{p} r^{\star} \\
I_{c} & I_{p} & 0 & r^{\star} & 0 \\
-\Phi_{2} & 0 & 0 & -\Phi_{1} & 1
\end{array}\right)\left(\begin{array}{c}
\mathrm{d} \tilde{c} \\
\mathrm{~d} \tilde{p} \\
\mathrm{~d} \bar{\lambda} \\
\mathrm{d} \tilde{k} \\
\mathrm{~d} \tilde{b}
\end{array}\right) \\
& =\left(\begin{array}{c}
0 \\
\mathrm{~d} g^{D}+\tilde{p} \mathrm{~d} g^{F} \\
\mathrm{~d} g^{D} \\
\mathrm{~d} b_{0}-\Phi_{1} \mathrm{~d} k_{0}-\Phi_{2} \mathrm{~d} s_{0}
\end{array}\right),\left(\begin{array}{c} 
\\
\end{array}\right)
\end{aligned}
$$


where $\left(\tilde{c}^{F}+g^{F}-r^{\star} \tilde{b}\right)=\tilde{X} / \tilde{p}$.

Before turning to long-run changes, we introduce some useful properties we will use later:

$$
\begin{aligned}
\left(\sigma+\mu_{1}\right)\left(\sigma+\mu_{2}\right) & =\sigma\left[\sigma+\left(\mu_{1}+\mu_{2}\right)\right]+\mu_{1} \mu_{2}>0, \\
\mu_{3} \mu_{4} & =\left(r^{\star}-\mu_{1}\right)\left(r^{\star}-\mu_{2}\right)=r^{\star}\left[r^{\star}-\left(\mu_{1}+\mu_{2}\right)\right]+\mu_{1} \mu_{2}>0 .
\end{aligned}
$$

Wa calculate the determinant $D$ which is given by:

$$
\begin{aligned}
D & \equiv F_{k k} \frac{p_{c}}{\tilde{p}}\left\{-\tilde{p} I_{p} r^{\star} \Phi_{2}+p_{c}\left[I_{p}+\left(1-\alpha_{c}\right)\left(\tilde{c}^{F}+g^{F}-r^{\star} \tilde{b}\right)\right]\right\}, \\
& \equiv F_{k k} \frac{p_{c}}{\tilde{p}}\left\{-\tilde{p} I_{p} r^{\star} \Phi_{2}+p_{c}\left[I_{p}+\left(1-\alpha_{c}\right) \frac{\tilde{X}}{\tilde{p}}\right]\right\}, \\
& =(-) \times(-)>0,
\end{aligned}
$$

where we used the market-clearing condition, i. e. $\tilde{c}^{F}+g^{F}-r^{\star} \tilde{b}=\tilde{X} / \tilde{p}$ (see eq (10b)). To determine the sign of the determinant $D$, we have rewritten the term in square brackets as follows by making use of properties (61):

$$
\begin{aligned}
& -\tilde{p} I_{p} r^{\star} \Phi_{2}+p_{c}\left[I_{p}+\left(1-\alpha_{c}\right) \frac{\tilde{X}}{\tilde{p}}\right] \\
= & p_{c}\left[I_{p}+\left(1-\alpha_{c}\right) \frac{\tilde{X}}{\tilde{p}}\right]\left\{\frac{\mu_{1} \mu_{2} \mu_{3} \mu_{4} \sigma\left(\sigma+r^{\star}\right)}{\sigma \mu_{3} \mu_{4}\left\{\mu_{1} \mu_{2}\left(\sigma+r^{\star}\right)+I_{p} \tilde{p} F_{k k}\left[\sigma+\left(\mu_{1}+\mu_{2}\right)\right]\right\}}\right. \\
& \left.+\frac{I_{p} \tilde{p} F_{k k}\left\{\sigma \mu_{3} \mu_{4}\left[\sigma+\left(\mu_{1}+\mu_{2}\right)\right]-r^{\star}\left[r^{\star}-\left(\mu_{1}+\mu_{2}\right)\right]\left(\sigma+\mu_{1}\right)\left(\sigma+\mu_{2}\right)\right\}}{\sigma \mu_{3} \mu_{4}\left\{\mu_{1} \mu_{2}\left(\sigma+r^{\star}\right)+I_{p} \tilde{p} F_{k k}\left[\sigma+\left(\mu_{1}+\mu_{2}\right)\right]\right\}}\right\}, \\
= & p_{c}\left[I_{p}+\left(1-\alpha_{c}\right) \frac{\tilde{X}}{\tilde{p}}\right] \mu_{1} \mu_{2}\left\{\frac{\left(\sigma+\mu_{1}\right)\left(\sigma+\mu_{2}\right) I_{p} \tilde{p} F_{k k}+\mu_{3} \mu_{4}\left[\sigma\left(\sigma+r^{\star}\right)-I_{p} \tilde{p} F_{k k}\right]}{\sigma \mu_{3} \mu_{4}\left\{\mu_{1} \mu_{2}\left(\sigma+r^{\star}\right)+I_{p} \tilde{p} F_{k k}\left[\sigma+\left(\mu_{1}+\mu_{2}\right)\right]\right\}}\right\}, \\
= & p_{c}\left[I_{p}+\left(1-\alpha_{c}\right) \frac{\tilde{X}}{\tilde{p}}\right] \mu_{1} \mu_{2}\left\{\frac{\left(\sigma+\mu_{1}\right)\left(\sigma+\mu_{2}\right) I_{p} \tilde{p} F_{k k}}{\sigma \mu_{3} \mu_{4}\left\{\mu_{1} \mu_{2}\left(\sigma+r^{\star}\right)+I_{p} \tilde{p} F_{k k}\left[\sigma+\left(\mu_{1}+\mu_{2}\right)\right]\right\}}\right. \\
+ & \left.\frac{\mu_{3} \mu_{4}\left\{\left(\sigma+\mu_{1}\right)\left(\sigma+\mu_{4}\right)-\left[\mu_{1} \mu_{4}+I_{p} \tilde{p} F_{k k}\right]\right\}}{\sigma \mu_{3} \mu_{4}\left\{\mu_{1} \mu_{2}\left(\sigma+r^{\star}\right)+I_{p} \tilde{p} F_{k k}\left[\sigma+\left(\mu_{1}+\mu_{2}\right)\right]\right\}}\right\}, \\
= & (-) \times\left\{\frac{(+)}{(+)}\right\}<0,
\end{aligned}
$$

and by computing some useful expressions:

$$
\begin{aligned}
& \sigma \mu_{3} \mu_{4}\left[\sigma+\left(\mu_{1}+\mu_{2}\right)\right]-r^{\star}\left[r^{\star}-\left(\mu_{1}+\mu_{2}\right)\right]\left(\sigma+\mu_{1}\right)\left(\sigma+\mu_{2}\right) \\
= & \mu_{1} \mu_{2}\left[\left(\sigma+\mu_{1}\right)\left(\sigma+\mu_{2}\right)-\mu_{3} \mu_{4}\right] \\
& \sigma\left(\sigma+r^{\star}\right)-I_{p} \tilde{p} F_{k k} \\
= & \left(\sigma+\mu_{1}\right)\left(\sigma+\mu_{4}\right)-\left[\mu_{1} \mu_{4}+I_{p} \tilde{p} F_{k k}\right]>0 .
\end{aligned}
$$




\section{Domestic Good $g^{D}$}

The long-run effects after an unanticipated permanent increase in government expenditure on the domestic good are obtained from the total differential of the equilibrium system (10) with respect to $g^{D}$ :

$$
\begin{aligned}
\frac{\mathrm{d} \tilde{c}}{\mathrm{~d} g^{D}} & =\frac{\mathrm{d} \tilde{s}}{\mathrm{~d} g^{D}}=-\frac{F_{k k}}{D} \frac{p_{c}}{\tilde{p}}\left(I_{p}+\frac{\tilde{X}}{\tilde{p}}\right)=-\frac{(+)}{(+)}<0, \\
\frac{\mathrm{d} \tilde{p}}{\mathrm{~d} g^{D}} & =-\frac{F_{k k}}{D} \frac{p_{c}}{\tilde{p}}\left(\tilde{p} r^{\star} \Phi_{2}-\alpha_{c} p_{c}\right) \gtrless 0, \\
\frac{\mathrm{d} \bar{\lambda}}{\mathrm{d} g^{D}} & =-\frac{F_{k k}}{D} \frac{p_{c} \bar{\lambda}\left(1-\alpha_{c}\right)}{\tilde{p}^{2}}\left(\tilde{p} r^{\star} \Phi_{2}-\alpha_{c} p_{c}\right)-\frac{F_{k k}}{D}\left[u_{c c}+\frac{\delta+2 \sigma}{\delta+\sigma} \Gamma\right]\left(I_{p}+\frac{\tilde{X}}{\tilde{p}}\right) \gtrless 0, \\
\frac{\mathrm{d} \tilde{k}}{\mathrm{~d} g^{D}} & =0, \\
\frac{\mathrm{d} \tilde{b}}{\mathrm{~d} g^{D}} & =-\frac{F_{k k}}{D} \frac{p_{c}}{\tilde{p}} \Phi_{2}\left(I_{p}+\frac{\tilde{X}}{\tilde{p}}\right)=-\frac{(+)}{(+)}<0,
\end{aligned}
$$

where the determinant $D>0$ is given by (65), $\Phi_{2}>0$ (see (52)), $I_{p}+\frac{\tilde{X}}{\tilde{p}}=$ $-\frac{\tilde{X}}{\tilde{p}}\left[\nu_{X}+\nu_{f} \frac{\tilde{p} \tilde{c}^{F}}{\tilde{X}}-1\right]<0$, and we have computed the following expression:

$$
\begin{aligned}
& \tilde{p} r^{\star} \Phi_{2}-\alpha_{c} p_{c} \\
= & \frac{p_{c} I_{p} \tilde{p} F_{k k}\left\{r^{\star}\left[r^{\star}-\left(\mu_{1}+\mu_{2}\right)\right]\left(\sigma+\mu_{1}\right)\left(\sigma+\mu_{2}\right)-\alpha_{c} \mu_{3} \mu_{4} \sigma\left[\sigma+\left(\mu_{1}+\mu_{2}\right)\right]\right\}}{\sigma \mu_{3} \mu_{4}\left\{\mu_{1} \mu_{2}\left(\sigma+r^{\star}\right)+I_{p} \tilde{p} F_{k k}\left[\sigma+\left(\mu_{1}+\mu_{2}\right)\right]\right\}} \\
+ & \frac{p_{c} \tilde{p} F_{k k}\left(1-\alpha_{c}\right) \frac{\tilde{X}}{\tilde{p}}\left(\sigma+\mu_{1}\right)\left(\sigma+\mu_{2}\right) r^{\star}\left[r^{\star}-\left(\mu_{1}+\mu_{2}\right)\right]-p_{c} \alpha_{c} \mu_{1} \mu_{2} \mu_{3} \mu_{4} \sigma\left(\sigma+r^{\star}\right)}{\sigma \mu_{3} \mu_{4}\left\{\mu_{1} \mu_{2}\left(\sigma+r^{\star}\right)+I_{p} \tilde{p} F_{k k}\left[\sigma+\left(\mu_{1}+\mu_{2}\right)\right]\right\}} \\
= & \frac{p_{c} \tilde{p} F_{k k}\left(\sigma+\mu_{1}\right)\left(\sigma+\mu_{2}\right)\left[\left(1-\alpha_{c}\right)\left(\mu_{3} \mu_{4}-\mu_{1} \mu_{2}\right)\left(I_{p}+\frac{\tilde{X}}{\tilde{p}}\right)+\alpha_{c} \mu_{1} \mu_{2} I_{p}\right]}{\sigma \mu_{3} \mu_{4}\left\{\mu_{1} \mu_{2}\left(\sigma+r^{\star}\right)+I_{p} \tilde{p} F_{k k}\left[\sigma+\left(\mu_{1}+\mu_{2}\right)\right]\right\}} \\
- & \frac{p_{c} \alpha_{c} \mu_{1} \mu_{2} \mu_{3} \mu_{4}\left\{\left(\sigma+\mu_{1}\right)\left(\sigma+\mu_{4}\right)-\left[\mu_{1} \mu_{4}+I_{p} \tilde{p} F_{k k}\right]\right\}}{\sigma \mu_{3} \mu_{4}\left\{\mu_{1} \mu_{2}\left(\sigma+r^{\star}\right)+I_{p} \tilde{p} F_{k k}\left[\sigma+\left(\mu_{1}+\mu_{2}\right)\right]\right\}}, \\
= & \frac{(+)}{(+)}-\frac{(+)}{(+)} \gtrless 0,
\end{aligned}
$$

with $\left(\mu_{3} \mu_{4}-\mu_{1} \mu_{2}\right)>0$ and

$$
\begin{aligned}
& r^{\star}\left[r^{\star}-\left(\mu_{1}+\mu_{2}\right)\right]\left(\sigma+\mu_{1}\right)\left(\sigma+\mu_{2}\right)-\alpha_{c} \mu_{3} \mu_{4} \sigma\left[\sigma+\left(\mu_{1}+\mu_{2}\right)\right] \\
= & \left(\sigma+\mu_{1}\right)\left(\sigma+\mu_{2}\right)\left[\left(1-\alpha_{c}\right) \mu_{3} \mu_{4}-\mu_{1} \mu_{2}\right]+\alpha_{c} \mu_{1} \mu_{2} \mu_{3} \mu_{4}, \\
& \left(1-\alpha_{c}\right) \mu_{3} \mu_{4}\left(I_{p}+\frac{\tilde{X}}{\tilde{p}}\right)-\mu_{1} \mu_{2}\left[I_{p}+\left(1-\alpha_{c}\right) \frac{\tilde{X}}{\tilde{p}}\right] \\
= & \left(1-\alpha_{c}\right) r^{\star}\left[r^{\star}-\left(\mu_{1}+\mu_{2}\right)\right]\left(I_{p}+\frac{\tilde{X}}{\tilde{p}}\right)+\alpha_{c} \mu_{1} \mu_{2} I_{p}<0 .
\end{aligned}
$$


The sign of (66) is ambiguous leaving the directions of steady-state changes (65b) and (65c) undetermined.

The sign of $\frac{\mathrm{d} \bar{\lambda}}{\mathrm{d} g^{D}}$ is not clear-cut like in the standard model assuming TS preferences. Intuitively, an increase in lump-sum taxes is necessary to finance the additional public spending which in turn reduces unambiguously the disposable income of agents when expressed in terms of the domestic good, that is $r^{\star} \tilde{p} \tilde{b}+\tilde{Y}-T$. However, because the long-run real exchange rate appreciation raises the disposable income measured in terms of the foreign good by increasing domestic output net of taxes, the sign of the change in the equilibrium value of the marginal utility of wealth $\mathrm{d} \bar{\lambda}$ is not clear-cut, like in the time separability utility case. Since for all parametrization, the marginal utility jumps upward after a fiscal expansion, we assume now and thereafter that $\bar{\lambda}$ rises.

While the representative agent's disposable income may rise or fall when expressed in terms of the foreign good, due to a possibly real exchange rate's long-run decline, when it is measured in terms of the domestic good, that is $r^{\star} \tilde{p} \tilde{b}+F(\tilde{k})-T$, the disposable income of individuals falls unambiguously due to the rise in lump-sum taxes, $T$, and the long-run fall in traded bonds' holding, $\tilde{b}$, keeping in mind that the capital stock, $\tilde{k}$, remains unchanged in the longrun. Consequently, the marginal utility of wealth expressed in terms of the domestic good unambiguously rises after an unexpected permanent increase in government spending, that is:

$$
\begin{aligned}
\frac{\mathrm{d}}{\mathrm{d} p}\left[\frac{p_{c} \bar{\lambda}}{\tilde{p}}\right] & =\frac{p_{c}}{\tilde{p}} \frac{\mathrm{d} \bar{\lambda}}{\mathrm{d} g^{D}}-\frac{\bar{\lambda} p_{c}\left(1-\alpha_{c}\right)}{\tilde{p}^{2}} \frac{\mathrm{d} \tilde{p}}{\mathrm{~d} g^{D}} \\
& =-\frac{F_{k k}}{D}\left[u_{c c}+\frac{\delta+2 \sigma}{\delta+\sigma} \Gamma\right]\left(I_{p}+\frac{\tilde{X}}{\tilde{p}}\right)>0 .
\end{aligned}
$$

\section{Import Good $g^{F}$}

The long-run effects after an unanticipated permanent increase in government expenditure on the foreign good are obtained from the total differential of the equilibrium system (10) with 
respect to $g^{F}$ :

$$
\begin{aligned}
\frac{\mathrm{d} \tilde{c}}{\mathrm{~d} g^{F}} & =\frac{\mathrm{d} \tilde{s}}{\mathrm{~d} g^{F}}=-\frac{F_{k k}}{D} p_{c} I_{p}=-\frac{(+)}{(+)}<0, \\
\frac{\mathrm{d} \tilde{p}}{\mathrm{~d} g^{F}} & =-\frac{F_{k k}}{D}\left(1-\alpha_{c}\right)\left(p_{c}\right)^{2}=-\frac{(-)}{(+)}>0, \\
\frac{\mathrm{d} \bar{\lambda}}{\mathrm{d} g^{F}} & =-\frac{\tilde{p} F_{k k}}{D}\left\{\left[\frac{\left(1-\alpha_{c}\right) p_{c}}{\tilde{p}}\right]^{2} \bar{\lambda}+I_{p}\left[u_{c c}+\frac{\delta+2 \sigma}{\delta+\sigma} \Gamma\right]\right\}=-\frac{(-)}{(+)}>0, \\
\frac{\mathrm{d} \tilde{k}}{\mathrm{~d} g^{F}} & =0, \\
\frac{\mathrm{d} \tilde{b}}{\mathrm{~d} g^{F}} & =-\frac{F_{k k}}{D} p_{c} I_{p} \Phi_{2}=-\frac{(+)}{(+)}<0,
\end{aligned}
$$

where $D>0$ is given by (65).

\section{C.2 The Two-Step Solution Procedure}

In this section, we calculate the signs of the partial derivatives of the steady-state functions obtained in the first step of the two-step solution procedure by totally differentiating equations (10a) to (10b) without the intertemporal budget constraint, equation (10d). Totally differentiating equations (10a)-(10b) yields in matrix form:

$$
\begin{aligned}
& \left(\begin{array}{cccc}
{\left[u_{c c}+\left(\frac{\delta+2 \sigma}{\delta+\sigma}\right) \Gamma\right]} & \frac{p_{c} \bar{\lambda}\left(1-\alpha_{c}\right)}{\tilde{p}^{2}} & 0 & 0 \\
0 & 0 & F_{k k} & 0 \\
-p_{c} & -\left(\tilde{c}^{F}+g^{F}-r^{\star} \tilde{b}\right) & r^{\star} & \tilde{p} r^{\star} \\
I_{c} & I_{p} & r^{\star} & 0
\end{array}\right)\left(\begin{array}{c}
\mathrm{d} \tilde{c} \\
\mathrm{~d} \tilde{p} \\
\mathrm{~d} \tilde{k} \\
\mathrm{~d} \tilde{b}
\end{array}\right) \\
& =\left(\begin{array}{c}
\frac{p_{c}}{\tilde{p}} \mathrm{~d} \bar{\lambda} \\
0 \\
\mathrm{~d} g^{D}+\tilde{p} \mathrm{~d} g^{F} \\
\mathrm{~d} g^{D}
\end{array}\right),
\end{aligned}
$$

where $\left(\tilde{c}^{F}+g^{F}-r^{\star} \tilde{b}\right)=\tilde{X} / \tilde{p}$.

The determinant of the system matrix is given by:

$$
G \equiv F_{k k} \tilde{p} r^{\star}\left\{\left[u_{c c}+\left(\frac{\delta+2 \sigma}{\delta+\sigma}\right) \Gamma\right] I_{p}-\frac{p_{c} \bar{\lambda}\left(1-\alpha_{c}\right)}{\tilde{p}^{2}} I_{c}\right\}<0 .
$$

From system (68), we can calculate the partial derivatives of the following steady-state 
functions:

$$
\begin{aligned}
\tilde{k} & =F_{k}^{-1}\left(r^{\star}\right), \\
\tilde{s} & =\tilde{c}=m\left(\bar{\lambda}, g^{D}, g^{F}\right), \\
\tilde{p} & =p\left(\bar{\lambda}, g^{D}, g^{F}\right), \\
\tilde{b} & =b\left(\bar{\lambda}, g^{D}, g^{F}\right),
\end{aligned}
$$

with

$$
\begin{aligned}
m_{\bar{\lambda}} & \equiv \frac{\partial \tilde{c}}{\partial \bar{\lambda}}=\frac{F_{k k}}{G} I_{p} p_{c} r^{\star}=\frac{(+)}{(-)}<0 \\
m_{g^{D}} & \equiv \frac{\partial \tilde{c}}{\partial g^{D}}=-\frac{F_{k k}}{G} \frac{p_{c} \bar{\lambda}\left(1-\alpha_{c}\right)}{\tilde{p}} r^{\star}=\frac{(+)}{(-)}<0 \\
m_{g^{F}} & \equiv \frac{\partial \tilde{c}}{\partial g^{F}}=0 \\
p_{\bar{\lambda}} & \equiv \frac{\partial \tilde{p}}{\partial \bar{\lambda}}=-\frac{F_{k k}}{G} I_{c} p_{c} r^{\star}=\frac{(-)}{(-)}>0 \\
p_{g^{D}} & \equiv \frac{\partial \tilde{p}}{\partial g^{D}}=-\frac{F_{k k} \tilde{p} r^{\star}}{G}\left[u_{c c}+\left(\frac{\delta+2 \sigma}{\delta+\sigma}\right) \Gamma\right]=\frac{(+)}{(-)}<0 \\
p_{g^{F}} & \equiv \frac{\partial \tilde{p}}{\partial g^{F}}=0, \\
b_{\bar{\lambda}} & \equiv \frac{\partial \tilde{b}}{\partial \bar{\lambda}}=\frac{F_{k k}}{G} \frac{\left(p_{c}\right)^{2}}{\tilde{p}}\left[I_{p}+\left(1-\alpha_{c}\right) \frac{\tilde{X}}{\tilde{p}}\right]=\frac{(+)}{(-)}<0 \\
b_{g^{D}} & \equiv \frac{\partial \tilde{b}}{\partial g^{D}}=\frac{F_{k k}}{G}\left\{\left[u_{c c}+\left(\frac{\delta+2 \sigma}{\delta+\sigma}\right) \Gamma\right]\left[I_{p}+\left(1-\alpha_{c}\right) \frac{\tilde{X}}{\tilde{p}}\right]-\frac{p_{c}^{2} \bar{\lambda}\left(1-\alpha_{c}\right)}{\tilde{p}^{2}} \alpha_{c}\right\}, \\
& =(+) \times\{(+)-(+)\} \gtrless 0, \\
b_{g^{F}} & \equiv \frac{\partial \tilde{b}}{\partial g^{F}}=\frac{\tilde{p} F_{k k}}{G}\left\{\left[u_{c c}+\left(\frac{\delta+2 \sigma}{\delta+\sigma}\right) \Gamma\right] I_{p}-\frac{p_{c}^{2} \bar{\lambda}\left(1-\alpha_{c}\right)}{\tilde{p}^{2}} I_{c}\right\}, \\
& =(+) \times\{(+)-(-)\}>0,
\end{aligned}
$$

where $I_{c}<0, I_{p}<0$.

In the second step, we obtain the equilibrium value of the marginal utility of wealth by inserting the functions for $\tilde{c}=\tilde{s}, \tilde{p}$ and $\tilde{b}$ into the economy's intertemporal budget constraint (equation $(10 \mathrm{~d})$ ), keeping in mind that $\tilde{k}=$ constant:

$$
\left[b\left(\bar{\lambda}, g^{D}, g^{F}\right)-b_{0}\right]=\Phi_{1}\left(\tilde{k}-k_{0}\right)+\Phi_{2}\left(m\left(\bar{\lambda}, g^{D}, g^{F}\right)-s_{0}\right) .
$$

which yields to

$$
\bar{\lambda}=g\left(s_{0}, k_{0}, b_{0}, g^{D}, g^{F}\right)
$$


where partial derivatives are given by:

$$
\begin{gathered}
\frac{\mathrm{d} \bar{\lambda}}{\mathrm{d} g^{D}} \equiv \lambda_{g^{D}}=-\frac{b_{g^{D}}-\Phi_{2} m_{g^{D}}}{b_{\bar{\lambda}}-\Phi_{2} m_{\bar{\lambda}}}>0, \\
\frac{\mathrm{d} \bar{\lambda}}{\mathrm{d} g^{F}} \equiv \lambda_{g^{F}}=-\frac{b_{g^{F}}}{b_{\bar{\lambda}}-\Phi_{2} m_{\bar{\lambda}}}>0, \\
\frac{\mathrm{d} \bar{\lambda}}{\mathrm{d} b_{0}} \equiv \lambda_{n}=\frac{1}{b_{\bar{\lambda}}-\Phi_{2} m_{\bar{\lambda}}}<0, \\
\frac{\mathrm{d} \bar{\lambda}}{\mathrm{d} k_{0}} \equiv \lambda_{k}=-\frac{\Phi_{1}}{b_{\bar{\lambda}}-\Phi_{2} m_{\bar{\lambda}}}<0, \\
\frac{\mathrm{d} \bar{\lambda}}{\mathrm{d} s_{0}} \equiv \lambda_{s}=-\frac{\Phi_{2}}{b_{\bar{\lambda}}-\Phi_{2} m_{\bar{\lambda}}}>0,
\end{gathered}
$$

where we used the fact that $m_{g^{F}}=0$ and the signs of (74a) and (74b) correspond to these of $(65 c)$ and (67c).

\section{Graphical Constructions of Stable Manifolds}

We start the investigation of transitional dynamics by evaluating the constants after a government spending shock, $g^{j}$ (with $\left.i=d, f\right)$ :

$$
\begin{aligned}
& \frac{A_{1}}{\mathrm{~d} g^{j}}=-\frac{\omega_{3}^{2}}{\omega_{3}^{2}-\omega_{3}^{1}} \frac{\mathrm{d} \tilde{s}}{\mathrm{~d} g^{j}}>0, \quad i=d, f, \\
& \frac{A_{2}}{\mathrm{~d} g^{j}}=\frac{\omega_{3}^{1}}{\omega_{3}^{2}-\omega_{3}^{1}} \frac{\mathrm{d} \tilde{s}}{\mathrm{~d} g^{j}}<0, \quad i=d, f,
\end{aligned}
$$

where $\omega_{3}^{1}>0, \omega_{3}^{2}>0, \mathrm{~d} \tilde{s} / \mathrm{d} g^{j}<0($ for $i=d, f)$.

From (75), we deduce the following property:

$$
\frac{A_{2}}{\mathrm{~d} g^{j}}=-\frac{\omega_{3}^{1}}{\omega_{3}^{2}} \frac{A_{1}}{\mathrm{~d} g^{j}}, \quad i=g, f
$$

\section{D.1 Stable Manifold in the $(k, p)$-space}

The stable solution is given by the following system of equations

$$
\begin{aligned}
& k(t)-\tilde{k}=\omega_{3}^{1} A_{1} e^{\mu_{1} t}+\omega_{3}^{2} A_{2} e^{\mu_{2} t}, \\
& p(t)-\tilde{p}=\omega_{4}^{1} A_{1} e^{\mu_{1} t}+\omega_{4}^{2} A_{2} e^{\mu_{2} t} .
\end{aligned}
$$

Demarcation Lines 
Time derivatives of solutions (77a) and (77b) and elimination of $A_{1} e^{\mu_{1} t}$ and $A_{2} e^{\mu_{2} t}$ leads to a new linear differential equation system for $k(t)$ and $p(t)$

$$
\begin{aligned}
& \dot{k}(t)=\left[\frac{\mu_{1} \omega_{3}^{1} \omega_{4}^{2}-\mu_{2} \omega_{3}^{2} \omega_{4}^{1}}{\omega_{3}^{1} \omega_{4}^{2}-\omega_{3}^{2} \omega_{4}^{1}}\right](k(t)-\tilde{k})-\left[\frac{\omega_{3}^{1} \omega_{3}^{2}\left(\mu_{1}-\mu_{2}\right)}{\omega_{3}^{1} \omega_{4}^{2}-\omega_{3}^{2} \omega_{4}^{1}}\right](p(t)-\tilde{p}), \\
& \dot{p}(t)=\left[\frac{\omega_{4}^{1} \omega_{4}^{2}\left(\mu_{1}-\mu_{2}\right)}{\omega_{3}^{1} \omega_{4}^{2}-\omega_{3}^{2} \omega_{4}^{1}}\right](k(t)-\tilde{k})-\left[\frac{\mu_{1} \omega_{4}^{1} \omega_{3}^{2}-\mu_{2} \omega_{4}^{2} \omega_{3}^{1}}{\omega_{3}^{1} \omega_{4}^{2}-\omega_{3}^{2} \omega_{4}^{1}}\right](p(t)-\tilde{p}) .
\end{aligned}
$$

The new differential equation system has two stable roots $\mu_{1}<0$ and $\mu_{2}<0$ with $\mu_{1}<\mu_{2}<0$; hence the steady-state is a stable node in $(k, p)$-space.

From (78), we determine the slopes of demarcation lines:

$$
\begin{aligned}
\left.\frac{\mathrm{d} p}{\mathrm{~d} k}\right|_{\dot{k}=0} & =\frac{\mu_{1} \omega_{3}^{1} \omega_{4}^{2}-\mu_{2} \omega_{3}^{2} \omega_{4}^{1}}{\omega_{3}^{1} \omega_{3}^{2}\left(\mu_{1}-\mu_{2}\right)}=\frac{(-)}{(-)}>0, \\
\frac{\partial \dot{k}}{\partial p} & =-\frac{\omega_{3}^{1} \omega_{3}^{2}\left(\mu_{1}-\mu_{2}\right)}{\omega_{3}^{1} \omega_{4}^{2}-\omega_{3}^{2} \omega_{4}^{1}}=-\frac{(-)}{(+)}>0, \\
\left.\frac{\mathrm{d} p}{\mathrm{~d} k}\right|_{\dot{p}=0} & =\frac{\omega_{4}^{1} \omega_{4}^{2}\left(\mu_{1}-\mu_{2}\right)}{\mu_{1} \omega_{4}^{1} \omega_{3}^{2}-\mu_{2} \omega_{4}^{2} \omega_{3}^{1}}=\frac{(-)}{(0)}=-\infty \\
\frac{\partial \dot{p}}{\partial k} & =\frac{\omega_{4}^{1} \omega_{4}^{2}\left(\mu_{1}-\mu_{2}\right)}{\omega_{3}^{1} \omega_{4}^{2}-\omega_{3}^{2} \omega_{4}^{1}}=\frac{(-)}{(+)}<0,
\end{aligned}
$$

where $\mu_{1}-\mu_{2}<0$ and we have computed the following expressions:

$$
\begin{aligned}
& \mu_{1} \omega_{3}^{1} \omega_{4}^{2}-\mu_{2} \omega_{3}^{2} \omega_{4}^{1} \\
= & \frac{\tilde{p} F_{k k} \omega_{3}^{1} \omega_{3}^{2}\left(\mu_{1}+\mu_{2}\right)\left(\mu_{1}-\mu_{2}\right)}{\mu_{1} \mu_{2}}=\frac{(-)}{(+)}<0, \\
& \mu_{1} \omega_{4}^{1} \omega_{3}^{2}-\mu_{2} \omega_{4}^{2} \omega_{3}^{1}=0, \\
& \omega_{3}^{1} \omega_{4}^{2}-\omega_{3}^{2} \omega_{4}^{1} \\
= & \frac{\tilde{p} F_{k k} \omega_{3}^{1} \omega_{3}^{2}\left(\mu_{1}-\mu_{2}\right)}{\mu_{1} \mu_{2}}=\frac{(-)}{(+)}<0 .
\end{aligned}
$$

Making use of expressions (80), the system (78) may be rewritten as follows:

$$
\begin{aligned}
& \dot{k}(t)=\Theta_{1}(k(t)-\tilde{k})+\Theta_{2}(p(t)-\tilde{p}), \\
& \dot{p}(t)=\Theta_{3}(k(t)-\tilde{k})
\end{aligned}
$$


with

$$
\begin{aligned}
\Theta_{1} & =\frac{\mu_{1} \omega_{3}^{1} \omega_{4}^{2}-\mu_{2} \omega_{3}^{2} \omega_{4}^{1}}{\omega_{3}^{1} \omega_{4}^{2}-\omega_{3}^{2} \omega_{4}^{1}}=\frac{(-)}{(+)}<0, \\
\Theta_{2} & =\frac{\omega_{3}^{1} \omega_{3}^{2}\left(\mu_{1}-\mu_{2}\right)}{\omega_{3}^{1} \omega_{4}^{2}-\omega_{3}^{2} \omega_{4}^{1}}=-\frac{(-)}{(+)}>0, \\
\Theta_{3} & =\frac{\omega_{4}^{1} \omega_{4}^{2}\left(\mu_{1}-\mu_{2}\right)}{\omega_{3}^{1} \omega_{4}^{2}-\omega_{3}^{2} \omega_{4}^{1}}=\tilde{p} F_{k k}<0, \\
\Theta_{4} & =\frac{\mu_{1} \omega_{4}^{1} \omega_{3}^{2}-\mu_{2} \omega_{4}^{2} \omega_{3}^{1}}{\omega_{3}^{1} \omega_{4}^{2}-\omega_{3}^{2} \omega_{4}^{1}}=0,
\end{aligned}
$$

where the multipliers $\Theta_{i}(i=1,2,3,4)$ can be interpreted as partial speeds of adjustment towards the steady-state.

\section{Fiscal Expansion and Trajectories}

The slope of the trajectory at time $t$ is obtained by differentiating the system of equations (77) with respect to time $t$ and dividing the resulting expressions:

$$
\frac{\mathrm{d} p(t)}{\mathrm{d} K(t)}=\frac{\mu_{1} \omega_{4}^{1} A_{1} e^{\mu_{1} t}+\mu_{2} \omega_{4}^{2} A_{2} e^{\mu_{2} t}}{\mu_{1} \omega_{3}^{1} A_{1} e^{\mu_{1} t}+\mu_{2} \omega_{3}^{2} A_{2} e^{\mu_{2} t}}=\frac{\mu_{1} \omega_{4}^{1} A_{1} e^{\left(\mu_{1}-\mu_{2}\right) t}+\mu_{2} \omega_{4}^{2} A_{2}}{\mu_{1} \omega_{3}^{1} A_{1} e^{\left(\mu_{1}-\mu_{2}\right) t}+\mu_{2} \omega_{4}^{2} A_{2}} .
$$

The slope of the trajectory at the initial point can be calculated from (82) by setting $t=0$, substituting the constants $A_{1}$ and $A_{2}$ given by (37) and taking into account that $\mathrm{d} \tilde{k}=0$ :

$$
\begin{aligned}
\left.\left(\frac{\mathrm{d} p}{\mathrm{~d} k}\right)\right|_{t \rightarrow 0} & =\frac{\mu_{1} \omega_{4}^{1} A_{1} / \mathrm{d} g^{D}+\mu_{2} \omega_{4}^{2} A_{2} / \mathrm{d} g^{D}}{\mu_{1} \omega_{3}^{1} A_{1} / \mathrm{d} g^{D}-\mu_{2} \omega_{3}^{2} A_{2} / \mathrm{d} g^{D}}, \\
& =\frac{\mu_{1} \omega_{4}^{1} \omega_{3}^{2}-\mu_{2} \omega_{4}^{2} \omega_{3}^{1}}{\omega_{3}^{1} \omega_{3}^{2}\left(\mu_{1}-\mu_{2}\right)}=\frac{(0)}{(-)}=0 .
\end{aligned}
$$

Letting time tend towards infinity, i. e. $t \rightarrow \infty$, in equation (82), one obtains the slope of the trajectory approaching the steady-state:

$$
\left.\left(\frac{\mathrm{d} p}{\mathrm{~d} k}\right)\right|_{t \rightarrow \infty}=\frac{\omega_{4}^{2}}{\omega_{3}^{2}}=\frac{(+)}{(+)}>0
$$

We compare the slope of the demarcation line $\dot{k}=0$ with the slope of the trajectory approaching the steady-state in the $(k, p)$-space:

$$
\left.\left(\frac{\mathrm{d} p}{\mathrm{~d} k}\right)\right|_{t \rightarrow \infty}-\left.\frac{\mathrm{d} p}{\mathrm{~d} k}\right|_{\dot{k}=0}=-\frac{\mu_{2}\left(\omega_{3}^{1} \omega_{4}^{2}-\omega_{3}^{2} \omega_{4}^{1}\right)}{\omega_{3}^{1} \omega_{3}^{2}\left(\mu_{1}-\mu_{2}\right)}=-\frac{(-)}{(-)}<0
$$

where $\omega_{3}^{1} \omega_{4}^{2}-\omega_{3}^{2} \omega_{4}^{1}>0$ is given by (80c). From (94), we can deduce the following inequality:

$$
0<\left.\left(\frac{\mathrm{d} p}{\mathrm{~d} k}\right)\right|_{t \rightarrow \infty}<\left.\frac{\mathrm{d} p}{\mathrm{~d} k}\right|_{\dot{k}=0}
$$


We calculate the initial changes of the real exchange rate and the investment in physical capital.

\section{A Rise in Government Spending on the Domestic Good}

Setting $t=0$ into $(77 \mathrm{~b})$, differentiating with respect to $g^{D}$, and noting that $\frac{\mathrm{d} \tilde{k}}{\mathrm{~d} g^{D}}=0$, the real exchange rate appreciates initially following an unanticipated permanent rise in government spending on the domestic good:

$$
\begin{aligned}
\frac{\mathrm{d} p(0)}{\mathrm{d} g^{D}} & =\frac{\mathrm{d} \tilde{p}}{\mathrm{~d} g^{D}}+\omega_{4}^{1} \frac{A_{1}}{\mathrm{~d} g^{D}}+\omega_{4}^{2} \frac{A_{2}}{\mathrm{~d} g^{D}} \\
& =\frac{\mathrm{d} \tilde{p}}{\mathrm{~d} g^{D}}+\left[\frac{\omega_{3}^{1} \omega_{4}^{2}-\omega_{3}^{2} \omega_{4}^{1}}{\omega_{3}^{2}-\omega_{3}^{1}}\right] \frac{\mathrm{d} \tilde{s}}{\mathrm{~d} g^{D}}, \\
& =-\frac{F_{k k}}{D} \frac{p_{c}}{\tilde{p}}\left\{\left(\tilde{p} r^{\star} \Phi_{2}-\alpha_{c} p_{c}\right)+\left[\frac{\omega_{3}^{1} \omega_{4}^{2}-\omega_{3}^{2} \omega_{4}^{1}}{\omega_{3}^{2}-\omega_{3}^{1}}\right]\left(I_{p}+\frac{\tilde{X}}{\tilde{p}}\right)\right\}<0,
\end{aligned}
$$

where we have rewritten the term in square brackets to determine its sign:

$$
\begin{aligned}
& \left(\tilde{p} r^{\star} \Phi_{2}-\alpha_{c} p_{c}\right)+\left[\frac{\omega_{3}^{1} \omega_{4}^{2}-\omega_{3}^{2} \omega_{4}^{1}}{\omega_{3}^{2}-\omega_{3}^{1}}\right]\left(I_{p}+\frac{\tilde{X}}{\tilde{p}}\right) \\
= & \frac{\mu_{4} \omega_{3}^{1}\left[\mu_{3} \omega_{4}^{2}\left(I_{p}+\frac{\tilde{X}}{\tilde{p}}\right)+\tilde{p} r^{\star} N_{2}\right]-\mu_{3} \omega_{3}^{2}\left[\mu_{4} \omega_{4}^{1}\left(I_{p}+\frac{\tilde{X}}{\tilde{p}}\right)+\tilde{p} r^{\star} N_{1}\right]}{\mu_{3} \mu_{4}\left(\omega_{3}^{2}-\omega_{3}^{1}\right)} \\
- & \frac{\alpha_{c} p_{c} \mu_{3} \mu_{4}\left(\omega_{3}^{2}-\omega_{3}^{1}\right)}{\mu_{3} \mu_{4}\left(\omega_{3}^{2}-\omega_{3}^{1}\right)} \\
= & \frac{\tilde{p} F_{k k} \omega_{3}^{1} \omega_{3}^{2}\left(I_{p}+\frac{\tilde{X}}{\tilde{p}}\right)\left(\mu_{3}-\mu_{4}\right)+\tilde{p} r^{\star} p_{c}^{\prime}\left[\left(\frac{\sigma+\mu_{1}}{\sigma}\right) \mu_{3} \omega_{3}^{2}-\left(\frac{\sigma+\mu_{2}}{\sigma}\right) \mu_{4} \omega_{3}^{1}\right]}{\mu_{3} \mu_{4}\left(\omega_{3}^{2}-\omega_{3}^{1}\right)} \\
- & \frac{\alpha_{c} p_{c} \mu_{3} \mu_{4}\left(\omega_{3}^{2}-\omega_{3}^{1}\right)}{\mu_{3} \mu_{4}\left(\omega_{3}^{2}-\omega_{3}^{1}\right)} \\
= & \frac{p_{c} I_{c} \tilde{p} F_{k k}\left(\sigma+\mu_{1}\right)\left(\sigma+\mu_{2}\right)\left\{\alpha_{c} \mu_{3} \mu_{4}\left(\mu_{1}-\mu_{2}\right) I_{p}-\mu_{1} \mu_{2}\left(\mu_{3}-\mu_{4}\right)\left[I_{p}+\left(1-\alpha_{c}\right) \tilde{X} \tilde{\tilde{x}}\right]\right\}}{\sigma^{2}\left[\mu_{1} \mu_{4}+I_{p} \tilde{p} F_{k k}\right]\left[\mu_{2} \mu_{3}+I_{p} \tilde{p} F_{k k}\right] \mu_{3} \mu_{4}\left(\omega_{3}^{2}-\omega_{3}^{1}\right)} \\
- & \frac{\alpha_{c} p_{c} \mu_{3} \mu_{4} I_{c}\left(\mu_{1}-\mu_{2}\right) \sigma\left\{\mu_{1} \mu_{2}\left(\sigma+r^{\star}\right)+I_{p} \tilde{p} F_{k k}\left[\sigma+\left(\mu_{1}+\mu_{2}\right)\right]\right\}}{\sigma^{2}\left[\mu_{1} \mu_{4}+I_{p} \tilde{p} F_{k k}\right]\left[\mu_{2} \mu_{3}+I_{p} \tilde{p} F_{k k}\right] \mu_{3} \mu_{4}\left(\omega_{3}^{2}-\omega_{3}^{1}\right)} \\
= & -p_{c} I_{c} \mu_{1} \mu_{2}\left\{\frac{\tilde{p} F_{k k}\left(\sigma+\mu_{1}\right)\left(\sigma+\mu_{2}\right)\left(\mu_{3}-\mu_{4}\right)\left[I_{p}+\left(1-\alpha_{c}\right) \frac{\tilde{X}}{\tilde{p}}\right]}{\sigma^{2}\left[\mu_{1} \mu_{4}+I_{p} \tilde{p} F_{k k}\right]\left[\mu_{2} \mu_{3}+I_{p} \tilde{p} F_{k k}\right] \mu_{3} \mu_{4}\left(\omega_{3}^{2}-\omega_{3}^{1}\right)}\right. \\
= & \left.\frac{\alpha_{c} \mu_{3} \mu_{4}\left(\mu_{1}-\mu_{2}\right)\left\{\left[\mu_{1} \mu_{4}+I_{p} \tilde{p} F_{k k}\right]-\left(\sigma+\mu_{1}\right)\left(\sigma+\mu_{4}\right)\right\}}{\sigma^{2}\left[\mu_{1} \mu_{4}+I_{p} \tilde{p} F_{k k}\right]\left[\mu_{2} \mu_{3}+I_{p} \tilde{p} F_{k k}\right] \mu_{3} \mu_{4}\left(\omega_{3}^{2}-\omega_{3}^{1}\right)}\right\}, \\
= & (+) \times\left\{\frac{(-)}{(+)}-\frac{(+)}{(+)}\right\}<0,
\end{aligned}
$$

where we used the definition of $\Phi_{2}$ (see (50b)) and collected terms to obtain the first line; then 
we have evaluated the term in square brackets as follows to determine the second line:

$$
\begin{aligned}
& \mu_{3} \omega_{4}^{2}\left(I_{p}+\frac{\tilde{X}}{\tilde{p}}\right)+\tilde{p} r^{\star} N_{2} \\
= & -\left[\mu_{2} \omega_{4}^{2}\left(I_{p}+\frac{\tilde{X}}{\tilde{p}}\right)+\tilde{p} r^{\star} p_{c}^{\prime}\left(\frac{\sigma+\mu_{2}}{\sigma}\right)\right], \\
& \mu_{4} \omega_{4}^{1}\left(I_{p}+\frac{\tilde{X}}{\tilde{p}}\right)+\tilde{p} r^{\star} N_{1} \\
= & -\left[\mu_{1} \omega_{4}^{1}\left(I_{p}+\frac{\tilde{X}}{\tilde{p}}\right)+\tilde{p} r^{\star} p_{c}^{\prime}\left(\frac{\sigma+\mu_{1}}{\sigma}\right)\right], \\
& \left(\frac{\sigma+\mu_{1}}{\sigma}\right) \mu_{3} \omega_{3}^{2}-\left(\frac{\sigma+\mu_{2}}{\sigma}\right) \mu_{4} \omega_{3}^{1} \\
= & \frac{I_{c}\left(\sigma+\mu_{1}\right)\left(\sigma+\mu_{2}\right) I_{p} \tilde{p} F_{k k}\left(\mu_{1} \mu_{4}-\mu_{2} \mu_{3}\right)}{\sigma^{2}\left[\mu_{1} \mu_{4}+I_{p} \tilde{p} F_{k k}\right]\left[\mu_{2} \mu_{3}+I_{p} \tilde{p} F_{k k}\right]}=\frac{(+)}{(+)}>0, \\
& \tilde{p} F_{k k} I_{p}\left(\sigma+\mu_{1}\right)\left(\sigma+\mu_{2}\right)-\mu_{1} \mu_{2} \sigma\left(\sigma+r^{\star}\right)-I_{p} \tilde{p} F_{k k} \sigma\left[\sigma+\left(\mu_{1}+\mu_{2}\right)\right] \\
= & \mu_{1} \mu_{2}\left[I_{p} \tilde{p} F_{k k}-\sigma\left(\sigma+r^{\star}\right)\right] \\
= & \mu_{1} \mu_{2}\left\{\left[\mu_{1} \mu_{4}+I_{p} \tilde{p} F_{k k}\right]-\left(\sigma+\mu_{1}\right)\left(\sigma+\mu_{4}\right)\right\}<0 .
\end{aligned}
$$

Finally by developing the terms in square brackets, using the two last expressions, collecting terms, and rearranging, we obtain (88).

We turn to the investigation of transitional dynamics. Differentiate the stable solution for $k(t)$ and for $p(t)$ w.r.t time, one obtains the capital stock and the real exchange transitional dynamics:

$$
\begin{aligned}
& \dot{k}(t)=\underbrace{\mu_{1} \omega_{3}^{1} \frac{A_{1}}{\mathrm{~d} g^{D}} \mathrm{~d} g^{D}}_{(-)} e^{\mu_{1} t}+\underbrace{\mu_{2} \omega_{3}^{2} \frac{A_{2}}{\mathrm{~d} g^{D}} \mathrm{~d} g^{D}}_{(+)} e^{\mu_{2} t}, \\
& \dot{p}(t)=\underbrace{\mu_{1} \omega_{4}^{1} \frac{A_{1}}{\mathrm{~d} g^{D}} \mathrm{~d} g^{D}}_{(-)} e^{\mu_{2} t}+\underbrace{\mu_{2} \omega_{4}^{2} \frac{A_{2}}{\mathrm{~d} g^{D}} \mathrm{~d} g^{D}}_{(+)} e^{\mu_{2} t},
\end{aligned}
$$

where $\omega_{3}^{1}>0, \omega_{3}^{2}>0, \omega_{4}^{1}>0, \omega_{4}^{2}>0, \frac{A_{1}}{\mathrm{~d} g^{D}}>0, \frac{A_{2}}{\mathrm{~d} g^{D}}<0($ see $(75))$.

In a first place, we have to determine the initial changes of investment and the real exchange 
rate once it has jumped by setting $t=0$ into (89):

$$
\begin{aligned}
\frac{\mathrm{d} \dot{k}(0)}{\mathrm{d} g^{D}} & =\mu_{1} \omega_{3}^{1} \frac{A_{1}}{\mathrm{~d} g^{D}}+\mu_{2} \omega_{3}^{2} \frac{A_{2}}{\mathrm{~d} g^{D}}, \\
& =\left[\frac{\omega_{3}^{1} \omega_{3}^{2}}{\omega_{3}^{2}-\omega_{3}^{1}}\right]\left(\mu_{2}-\mu_{1}\right) \frac{\mathrm{d} \tilde{s}}{\mathrm{~d} g^{D}}<0, \\
\frac{\mathrm{d} \dot{p}(0)}{\mathrm{d} g^{D}} & =\mu_{1} \omega_{4}^{1} \frac{A_{1}}{\mathrm{~d} g^{D}}+\mu_{2} \omega_{4}^{2} \frac{A_{2}}{\mathrm{~d} g^{D}}, \\
& =-\left[\frac{\mu_{1} \omega_{4}^{1} \omega_{3}^{2}-\mu_{2} \omega_{4}^{2} \omega_{3}^{1}}{\omega_{3}^{2}-\omega_{3}^{1}}\right] \frac{\mathrm{d} \tilde{s}}{\mathrm{~d} g^{D}}=0,
\end{aligned}
$$

where we have substituted the expressions of constants given by (75) and made use of (80b) to determine (90b).

In a second place, we investigate in more details the condition of the non-monotonic adjustment of the investment in physical capital. More specifically, we must determine wether there exists a critical value of time, $t=\hat{t}>0$, such that investment, i. e. $I(\hat{t})=\dot{k}(\hat{t})=0$. Solving the first line of (90a), we obtain:

$$
\hat{t}=\frac{1}{\mu_{1}-\mu_{2}} \ln \left[-\frac{\mu_{2} \omega_{3}^{2} A_{2} / \mathrm{d} g^{D}}{\mu_{1} \omega_{3}^{1} A_{1} / \mathrm{d} g^{D}}\right]
$$

where $\mu_{1}-\mu_{2}<0$ and the necessary condition for $\hat{t}>0$ corresponds to:

$$
0<-\frac{\mu_{2} \omega_{3}^{2} A_{2} / \mathrm{d} g^{D}}{\mu_{1} \omega_{3}^{1} A_{1} / \mathrm{d} g^{D}}<1 \quad \Leftrightarrow \quad \frac{\mathrm{d} \dot{k}(0)}{\mathrm{d} g^{D}}<0
$$

If the condition (92) holds, the stock of physical capital initially decreases before reaching a turning point at time $\hat{t}$. Subsequently, investment turns to be positive and the stock of capital goods increases towards its unchanged steady-state level.

Regarding the transitional path followed by the real exchange rate, it is convenient to rewrite (89b) by using the fact that $\omega_{4}^{i}=\frac{\tilde{p} F_{k k}}{\mu_{i}} \omega_{3}^{i}$ and property $(76)$ :

$$
\dot{p}(t)=-\tilde{p} F_{k k} \omega_{3}^{1} \frac{A_{1}}{\mathrm{~d} g^{D}} \mathrm{~d} g^{D}\left(1-e^{\left(\mu_{1}-\mu_{2}\right) t}\right) e^{\mu_{2} t}>0 .
$$

From (87) and (93), we deduce that the relative price of the foreign good jumps initially downward and then the real exchange rate depreciates along a stable monotonic transitional path.

\section{A Rise in Government Spending on the Foreign Good}


Setting $t=0$ into $(77 \mathrm{~b})$, differentiating with respect to $g^{F}$, and noting that $\frac{\mathrm{d} \tilde{k}}{\mathrm{~d} g^{F}}=0$, the real exchange rate depreciates on impact following an unanticipated permanent rise in government spending on the foreign good:

$$
\begin{aligned}
\frac{\mathrm{d} p(0)}{\mathrm{d} g^{F}} & =\frac{\mathrm{d} \tilde{p}}{\mathrm{~d} g^{F}}+\omega_{4}^{1} \frac{A_{1}}{\mathrm{~d} g^{F}}+\omega_{4}^{2} \frac{A_{2}}{\mathrm{~d} g^{F}}, \\
& =-\left[\frac{I_{c}\left(\omega_{3}^{2}-\omega_{3}^{1}\right)+\left(\omega_{3}^{1} \omega_{4}^{2}-\omega_{3}^{2} \omega_{4}^{1}\right) I_{p}}{I_{p}\left(\omega_{3}^{2}-\omega_{3}^{1}\right)}\right] \frac{\mathrm{d} \tilde{s}}{\mathrm{~d} g^{F}}, \\
& =\left\{\frac{\left(I_{c}\right)^{2}\left(\mu_{1}-\mu_{2}\right) \mu_{1} \mu_{2}\left[I_{p} \tilde{p} F_{k k}-\sigma\left(\sigma+r^{\star}\right)\right]}{(\sigma)^{2}\left[\mu_{1} \mu_{4}+I_{p} \tilde{p} F_{k k}\right]\left[\mu_{2} \mu_{3}+I_{p} \tilde{p} F_{k k}\right] I_{p}\left(\omega_{3}^{2}-\omega_{3}^{1}\right)}\right\} \frac{\mathrm{d} \tilde{s}}{\mathrm{~d} g^{F}}, \\
& =\left\{\frac{I_{c} \mu_{1} \mu_{2}\left\{\left[\mu_{1} \mu_{4}+I_{p} \tilde{p} F_{k k}\right]-\left(\sigma+\mu_{1}\right)\left(\sigma+\mu_{4}\right)\right\}}{\sigma I_{p}\left\{\mu_{1} \mu_{2}(\sigma+r *)+I_{p} \tilde{p} F_{k k}\left[\sigma+\left(\mu_{1}+\mu_{2}\right)\right]\right\}}\right\} \frac{\mathrm{d} \tilde{s}}{\mathrm{~d} g^{F}}=\frac{(+)}{(-)} \times(-)>0,
\end{aligned}
$$

where we used the following relationship

$$
\frac{\mathrm{d} \tilde{p}}{\mathrm{~d} g^{F}}=-\frac{I_{c}}{I_{p}} \frac{\mathrm{d} \tilde{s}}{\mathrm{~d} g^{F}}
$$

and $(80 \mathrm{c})$ to obtain the second line. Then we have rewritten the following expressions:

$$
\begin{aligned}
& I_{c}\left(\omega_{3}^{2}-\omega_{3}^{1}\right)+\left(\omega_{3}^{1} \omega_{4}^{2}-\omega_{3}^{2} \omega_{4}^{1}\right) I_{p} \\
= & \frac{\left(I_{c}\right)^{2}\left(\mu_{1}-\mu_{2}\right) \mu_{1} \mu_{2}\left[I_{p} \tilde{p} F_{k k}-\sigma\left(\sigma+r^{\star}\right)\right]}{(\sigma)^{2}\left[\mu_{1} \mu_{4}+I_{p} \tilde{p} F_{k k}\right]\left[\mu_{2} \mu_{3}+I_{p} \tilde{p} F_{k k}\right]}=\frac{(+)}{(+)}>0, \\
& I_{p} \tilde{p} F_{k k}-\sigma\left(\sigma+r^{\star}\right) \\
= & {\left[\mu_{1} \mu_{4}+I_{p} \tilde{p} F_{k k}\right]-\left(\sigma+\mu_{1}\right)\left(\sigma+\mu_{4}\right)<0, }
\end{aligned}
$$

and substituted the expression of $\left(\omega_{3}^{2}-\omega_{3}^{1}\right)$ given by (46) to obtain (94).

From (94) and (67b), the real exchange rate depreciates (i. e. $p$ rises) in the short-run and the long-run. We have now to evaluate if the real exchange rate initially overshoots or not its steady-state value. Substituting (95) into (94), we have to evaluate if the following inequality holds or not :

$$
0<-\frac{\mu_{1} \mu_{2}\left\{\left[\mu_{1} \mu_{4}+I_{p} \tilde{p} F_{k k}\right]-\left(\sigma+\mu_{1}\right)\left(\sigma+\mu_{4}\right)\right\}}{\sigma\left\{\mu_{1} \mu_{2}(\sigma+r *)+I_{p} \tilde{p} F_{k k}\left[\sigma+\left(\mu_{1}+\mu_{2}\right)\right]\right\}}<1
$$

By making use of (47), it is straightforward to show that the inequality above holds since by rearranging terms, we get

$$
\left(\sigma+\mu_{1}\right)\left\{\mu_{2}\left[\mu_{1} \mu_{4}+I_{p} \tilde{p} F_{k k}\right]+\left[-\mu_{1}\left(\mu_{2}\right)^{2}+\sigma I_{p} \tilde{p} F_{k k}\right]\right\}>0 .
$$


We turn to the investigation of transitional dynamics following a rise in government spending on the foreign good. Differentiate the stable solution for $k(t)$ and for $p(t)$ w.r.t time, one obtains the capital stock and the real exchange transitional dynamics:

$$
\begin{aligned}
& \dot{k}(t)=\underbrace{\mu_{1} \omega_{3}^{1} \frac{A_{1}}{\mathrm{~d} g^{F}} \mathrm{~d} g^{F}}_{(-)} e^{\mu_{1} t}+\underbrace{\mu_{2} \omega_{3}^{2} \frac{A_{2}}{\mathrm{~d} g^{F}} \mathrm{~d} g^{F}}_{(+)} e^{\mu_{2} t}, \\
& \dot{p}(t)=\underbrace{\mu_{1} \omega_{4}^{1} \frac{A_{1}}{\mathrm{~d} g^{F}} \mathrm{~d} g^{F}}_{(-)} e^{\mu_{2} t}+\underbrace{\mu_{2} \omega_{4}^{2} \frac{A_{2}}{\mathrm{~d} g^{F}} \mathrm{~d} g^{F}}_{(+)} e^{\mu_{2} t},
\end{aligned}
$$

where $\omega_{3}^{1}>0, \omega_{3}^{2}>0, \omega_{4}^{1}>0, \omega_{4}^{2}>0, \frac{A_{1}}{\mathrm{~d} g^{F}}>0, \frac{A_{2}}{\mathrm{~d} g^{F}}<0($ see $(75))$.

In a first place, we have to determine the initial changes of investment and the real exchange rate once it has jumped by setting $t=0$ into (89):

$$
\begin{aligned}
\frac{\mathrm{d} \dot{k}(0)}{\mathrm{d} g^{F}} & =\mu_{1} \omega_{3}^{1} \frac{A_{1}}{\mathrm{~d} g^{F}}+\mu_{2} \omega_{3}^{2} \frac{A_{2}}{\mathrm{~d} g^{F}}, \\
& =\left[\frac{\omega_{3}^{1} \omega_{3}^{2}}{\omega_{3}^{2}-\omega_{3}^{1}}\right]\left(\mu_{2}-\mu_{1}\right) \frac{\mathrm{d} \tilde{s}}{\mathrm{~d} g^{F}}<0, \\
\frac{\mathrm{d} \dot{p}(0)}{\mathrm{d} g^{D}} & =\mu_{1} \omega_{4}^{1} \frac{A_{1}}{\mathrm{~d} g^{F}}+\mu_{2} \omega_{4}^{2} \frac{A_{2}}{\mathrm{~d} g^{F}}, \\
& =-\left[\frac{\mu_{1} \omega_{4}^{1} \omega_{3}^{2}-\mu_{2} \omega_{4}^{2} \omega_{3}^{1}}{\omega_{3}^{2}-\omega_{3}^{1}}\right] \frac{\mathrm{d} \tilde{s}}{\mathrm{~d} g^{F}}=0,
\end{aligned}
$$

where we have substituted the expressions of constants given by (75) and made use of (80b) to determine $(99 \mathrm{~b})$.

In a second place, we investigate in more details the condition of the non-monotonic adjustment of the investment in physical capital. More specifically, we must determine wether there exists a critical value of time, $t=\hat{t}>0$, such that investment reaches a turning point, i. e. $I(\hat{t})=\dot{k}(\hat{t})=0$. Solving (98a), we obtain:

$$
\hat{t}=\frac{1}{\mu_{1}-\mu_{2}} \ln \left[-\frac{\mu_{2} \omega_{3}^{2} A_{2} / \mathrm{d} g^{F}}{\mu_{1} \omega_{3}^{1} A_{1} / \mathrm{d} g^{F}}\right],
$$

where $\mu_{1}-\mu_{2}<0$ and the necessary condition for $\hat{t}>0$ corresponds to:

$$
0<-\frac{\mu_{2} \omega_{3}^{2} A_{2} / \mathrm{d} g^{F}}{\mu_{1} \omega_{3}^{1} A_{1} / \mathrm{d} g^{F}}<1 \quad \Leftrightarrow \quad \frac{\mathrm{d} \dot{k}(0)}{\mathrm{d} g^{F}}<0
$$

If the condition (101) holds, the stock of physical capital initially decreases before reaching a turning point at time $\hat{t}$. Subsequently, investment turns to be positive and the stock of capital goods increases towards its unchanged steady-state level. 
Finally, we have to determine the condition according to which the real exchange rate transitional path displays a non-monotonic pattern. We have to evaluate wether there exists a critical value of time, $t=\bar{t}>0$, such that the relative price of the foreign good reaches a turning point, i. e. $\dot{p}(\bar{t})=\dot{p}(\bar{t})=0$. Solving (98b), we obtain:

$$
\bar{t}=\frac{1}{\mu_{1}-\mu_{2}} \ln \left[-\frac{\mu_{2} \omega_{4}^{2} A_{2} / \mathrm{d} g^{F}}{\mu_{1} \omega_{4}^{1} A_{1} / \mathrm{d} g^{F}}\right]
$$

where $\mu_{1}-m u_{2}<0$ and the necessary condition for $\bar{t}>0$ corresponds to:

$$
0<-\frac{\mu_{2} \omega_{4}^{2} A_{2} / \mathrm{d} g^{F}}{\mu_{1} \omega_{4}^{1} A_{1} / \mathrm{d} g^{F}}<1 \quad \Leftrightarrow \quad \frac{\mathrm{d} \dot{p}(0)}{\mathrm{d} g^{F}}<0
$$

Since $\dot{p}(0)=0$ (and $\dot{p}(t)>0$ ), the condition (103) does not hold, and the real exchange rate adjusts monotonically.

Regarding the transitional path followed by the real exchange rate, it is convenient to rewrite (98b) by using the fact that $\omega_{4}^{i}=\frac{\tilde{p} F_{k k}}{\mu_{i}} \omega_{3}^{i}$ and property (76):

$$
\dot{p}(t)=-\tilde{p} F_{k k} \omega_{3}^{1} \frac{A_{1}}{\mathrm{~d} g^{F}} \mathrm{~d} g^{F}\left(1-e^{\left(\mu_{1}-\mu_{2}\right) t}\right) e^{\mu_{2} t}>0 .
$$

From (94) and (104), we deduce that the relative price of the foreign good jumps initially upward and then the real exchange rate depreciates along a stable monotonic transitional path.

\section{D.2 Stable Manifold in the $(k, b)$-space}

The stable solution is given by the following system of equations:

$$
\begin{aligned}
k(t)-\tilde{k} & =\omega_{3}^{1} A_{1} e^{\mu_{1} t}+\omega_{3}^{2} A_{2} e^{\mu_{2} t} \\
b(t)-\tilde{b} & =\frac{N_{1} A_{1}}{\mu_{1}-r^{\star}} e^{\mu_{1} t}+\frac{N_{2} A_{2}}{\mu_{2}-r^{\star}} e^{\mu_{2} t}
\end{aligned}
$$

\section{Demarcation Lines}

Time derivatives of solutions (105a) and (105b) and elimination of $A_{1} e^{\mu_{1} t}$ and $A_{2} e^{\mu_{2} t}$ leads 
to a new linear differential equation system for $k(t)$ and $b(t)$

$$
\begin{aligned}
\dot{k}(t)= & \frac{\left(\mu_{1}-r^{\star}\right) \mu_{1} \omega_{3}^{1} N_{2}-\left(\mu_{2}-r^{\star}\right) \mu_{2} \omega_{3}^{2} N_{1}}{\left(\mu_{1}-r^{\star}\right)\left(\mu_{2}-r^{\star}\right) \Delta^{k}}(k(t)-\tilde{k}) \\
& -\left[\frac{\omega_{3}^{1} \omega_{3}^{2}\left(\mu_{1}-\mu_{2}\right)}{\Delta^{k}}\right](b(t)-\tilde{b}), \\
\dot{b}(t)= & \frac{N_{1} N_{2}\left(\mu_{1}-\mu_{2}\right)}{\left(\mu_{1}-r^{\star}\right)\left(\mu_{2}-r^{\star}\right) \Delta^{k}}(k(t)-\tilde{k}) \\
& -\left[\frac{\mu_{1}\left(\mu_{2}-r^{\star}\right) \omega_{3}^{2} N_{1}-\mu_{2}\left(\mu_{1}-r^{\star}\right) \omega_{3}^{1} N_{2}}{\left(\mu_{1}-r^{\star}\right)\left(\mu_{2}-r^{\star}\right) \Delta^{k}}\right](b(t)-\tilde{b}),
\end{aligned}
$$

where

$$
\Delta^{k}=\frac{\left(\mu_{1}-r^{\star}\right) \omega_{3}^{1} N_{2}-\left(\mu_{2}-r^{\star}\right) \omega_{3}^{2} N_{1}}{\left(\mu_{1}-r^{\star}\right)\left(\mu_{2}-r^{\star}\right)}=\frac{(-)}{(+)}<0
$$

The new differential equation system has two stable roots $\mu_{1}<0$ and $\mu_{2}<0$ with $\mu_{1}<\mu_{2}<0$; hence the steady-state is a stable node in $(k, b)$-space.

From (106), we determine the slopes of demarcation lines:

$$
\begin{aligned}
\left.\frac{\mathrm{d} b}{\mathrm{~d} k}\right|_{\dot{k}=0} & =\frac{\mu_{1}\left(\mu_{1}-r^{\star}\right) \omega_{3}^{1} N_{2}-\mu_{2}\left(\mu_{2}-r^{\star}\right) \omega_{3}^{2} N_{1}}{\left(\mu_{1}-r^{\star}\right)\left(\mu_{2}-r^{\star}\right) \omega_{3}^{1} \omega_{3}^{2}\left(\mu_{1}-\mu_{2}\right)}=\frac{(?)}{(-)} \lessgtr 0, \\
\frac{\partial \dot{k}}{\partial n} & =-\frac{\left(\mu_{1}-r^{\star}\right)\left(\mu_{2}-r^{\star}\right) \omega_{3}^{1} \omega_{3}^{2}\left(\mu_{1}-\mu_{2}\right)}{\left(\mu_{1}-r^{\star}\right) \omega_{3}^{1} N_{2}-\left(\mu_{2}-r^{\star}\right) \omega_{3}^{2} N_{1}}=-\frac{(-)}{(-)}<0, \\
\left.\frac{\mathrm{d} b}{\mathrm{~d} k}\right|_{\dot{b}=0} & =\frac{N_{1} N_{2}\left(\mu_{1}-\mu_{2}\right)}{\mu_{1}\left(\mu_{2}-r^{\star}\right) \omega_{3}^{2} N_{1}-\mu_{2}\left(\mu_{1}-r^{\star}\right) \omega_{3}^{1} N_{2}}=\frac{(?)}{(-)} \lessgtr 0, \\
\frac{\partial \dot{b}}{\partial k} & =\frac{N_{1} N_{2}\left(\mu_{1}-\mu_{2}\right)}{\left(\mu_{1}-r^{\star}\right) \omega_{3}^{1} N_{2}-\left(\mu_{2}-r^{\star}\right) \omega_{3}^{2} N_{1}}=\frac{(?)}{(-)} \lessgtr 0,
\end{aligned}
$$

$\left(\mu_{1}-r^{\star}\right) \omega_{3}^{1} N_{2}-\left(\mu_{2}-r^{\star}\right) \omega_{3}^{2} N_{1}<0$ (see (48)). Several signs of expressions remain undetermined. The numerical analysis indicate that $N_{1}<0$ and $N_{2}>0$ for the benchmark parametrization, although $N_{2}$ may change of sign by assuming a small $\phi$ (higher but close to unity). 
We have computed several expressions as follows:

$$
\begin{aligned}
& \mu_{1}\left(\mu_{1}-r^{\star}\right) \omega_{3}^{1} N_{2}-\mu_{2}\left(\mu_{2}-r^{\star}\right) \omega_{3}^{2} N_{1} \\
= & \frac{\left(\mu_{1}-\mu_{2}\right) \omega_{3}^{1} \omega_{3}^{2}}{\mu_{1} \mu_{2} I_{c}} \frac{p_{c}}{\tilde{p}}\left\{\tilde{p} F_{k k}\left(I_{p}+\left(1-\alpha_{c}\right) \frac{\tilde{X}}{\tilde{p}}\right) \mu_{2}\left(\mu_{1}-\mu_{3}\right)\right. \\
& \left.-\mu_{1} \mu_{4}\left[\tilde{p} F_{k k}\left(I_{p}+\left(1-\alpha_{c}\right) \frac{\tilde{X}}{\tilde{p}}\right)+\alpha_{c} \mu_{2} \mu_{3}\right]\right\}=(+) \times[(+)+(?)] \gtrless 0, \\
& \mu_{1}\left(\mu_{2}-r^{\star}\right) \omega_{3}^{2} N_{1}-\mu_{2}\left(\mu_{1}-r^{\star}\right) \omega_{3}^{1} N_{2} \\
= & \frac{p_{c} I_{c}\left(\sigma+\mu_{1}\right)\left(\sigma+\mu_{2}\right) \mu_{1} \mu_{2}\left(\mu_{2}-\mu_{1}\right)\left[\tilde{p} F_{k k}\left(I_{p}+\left(1-\alpha_{c}\right) \frac{\tilde{X}}{\tilde{p}}\right)+\alpha_{c} \mu_{3} \mu_{4}\right]}{\sigma^{2}\left[\mu_{1} \mu_{4}+I_{p} \tilde{p} F_{k k}\right]\left[\mu_{2} \mu_{3}+I_{p} \tilde{p} F_{k k}\right]}, \\
= & \frac{(-)}{(+)}<0,
\end{aligned}
$$

where $\left[\left(\mu_{2}\right)^{2} \mu_{3}-\left(\mu_{1}\right)^{2} \mu_{4}\right]<0$ (see inequality (23)). To determine expression (109a), we used the fact that $\mu_{1}^{3}-\mu_{2}^{3}=\left(\mu_{1}-\mu_{2}\right)\left[\left(\mu_{1}+\mu_{2}\right)^{2}-\mu_{1} \mu_{2}\right]$ and $\left(\mu_{1}^{3}-\mu_{2}^{3}\right)-r^{\star}\left(\mu_{1}^{2}-\mu_{2}^{2}\right)=$ $\left(\mu_{1}-\mu_{2}\right)\left[\mu_{1} \mu_{2}-\left(\mu_{1} \mu_{4}+\mu_{2} \mu_{3}\right)\right]$. According to equation (50b), if $N_{2}>0$, then $\left[\tilde{p} F_{k k}\left(I_{p}+\left(1-\alpha_{c}\right) \frac{\tilde{X}}{\tilde{p}}\right)+\alpha_{c} \mu_{2} \mu_{3}\right]>0$ and equation (109a) is positive. Otherwise, if $N_{2}<0$, the sign of (109a) remains ambiguous.

\section{Fiscal Expansion and Trajectories}

The slope of the trajectory at time $t$ is obtained by differentiating the system of equations (105) with respect to time $t$ and dividing the resulting expressions:

$$
\frac{\mathrm{d} b(t)}{\mathrm{d} k(t)}=\frac{\mu_{1} \frac{N_{1} A_{1}}{\mu_{1}-r^{\star}} e^{\mu_{1} t}+\mu_{2} \frac{N_{2} A_{2}}{\mu_{2}-r^{\star}} e^{\mu_{2} t}}{\mu_{1} \omega_{3}^{1} A_{1} e^{\mu_{1} t}+\mu_{2} \omega_{3}^{2} A_{2} e^{\mu_{2} t}}=\frac{\mu_{1} \frac{N_{1} A_{1}}{\mu_{1}-r^{\star}} e^{\left(\mu_{1}-\mu_{2}\right) t}+\mu_{2} \frac{N_{2} A_{2}}{\mu_{2}-r^{\star}}}{\mu_{1} \omega_{3}^{1} A_{1} e^{\left(\mu_{1}-\mu_{2}\right) t}+\mu_{2} \omega_{3}^{2} A_{2}} .
$$

The slope of the trajectory at the initial point can be calculated from (110) by setting $t=0$, and substituting the constants $A_{1}$ and $A_{2}$ given by (75):

$$
\begin{aligned}
\left.\left(\frac{\mathrm{d} b}{\mathrm{~d} k}\right)\right|_{t \rightarrow 0} & =\frac{\mu_{1} \frac{N_{1}}{\mu_{1}-r^{\star}} \frac{A_{1}}{\mathrm{~d} g^{D}}+\mu_{2} \frac{N_{2}}{\mu_{2}-r^{\star}} \frac{A_{2}}{\mathrm{~d} g^{D}}}{\mu_{1} \omega_{3}^{1} \frac{A_{1}}{\mathrm{~d} g^{D}}+\mu_{2} \omega_{3}^{2} \frac{A_{2}}{\mathrm{~d} g^{D}}}, \\
& =-\frac{\mu_{1}\left(\mu_{2}-r^{\star}\right) \omega_{3}^{2} N_{1}-\mu_{2}\left(\mu_{1}-r^{\star}\right) \omega_{3}^{1} N_{2}}{\left(\mu_{1}-r^{\star}\right)\left(\mu_{2}-r^{\star}\right) \omega_{3}^{1} \omega_{3}^{2}\left(\mu_{2}-\mu_{1}\right)} \\
& =-\frac{(-)}{(+)}>0,
\end{aligned}
$$

where we have substituted the expressions of constants (see (75)). The term located in the numerator is given by (109b).

Letting time tend towards infinity, i. e. $t \rightarrow \infty$, in equation (110), and keeping in mind 
that $\mu_{1}-\mu_{2}<0$, one obtains the slope of the trajectory approaching the steady-state:

$$
\left.\left(\frac{\mathrm{d} b}{\mathrm{~d} k}\right)\right|_{t \rightarrow \infty}=\frac{N_{2}}{\left(\mu_{2}-r^{\star}\right) \omega_{3}^{2}}=\frac{(?)}{(-)} \lessgtr 0 .
$$

Finally, it is convenient to compare the slope of the demarcation line $\dot{k}=0$ with the slope of the trajectory approaching the steady-state in the $(k, b)$ space:

$$
\left.\frac{\mathrm{d} b}{\mathrm{~d} k}\right|_{\dot{k}=0}-\left.\left(\frac{\mathrm{d} b}{\mathrm{~d} k}\right)\right|_{t \rightarrow \infty}=-\frac{\mu_{2}\left[\left(\mu_{2}-r^{\star}\right) \omega_{3}^{2} N_{1}-\left(\mu_{1}-r^{\star}\right) \omega_{3}^{1} N_{2}\right]}{\left(\mu_{1}-r^{\star}\right)\left(\mu_{2}-r^{\star}\right) \omega_{3}^{1} \omega_{3}^{2}\left(\mu_{1}-\mu_{2}\right)}=-\frac{(-)}{(-)}<0,
$$

where $\mu_{1}-\mu_{2}<0$ and $\left(\mu_{2}-r^{\star}\right) \omega_{3}^{2} N_{1}-\left(\mu_{1}-r^{\star}\right) \omega_{3}^{1} N_{2}>0$ is given by (48). From (113), we can deduce the following inequality:

$$
\left.\frac{\mathrm{d} b}{\mathrm{~d} k}\right|_{\dot{k}=0}<\left.\left(\frac{\mathrm{d} b}{\mathrm{~d} k}\right)\right|_{t \rightarrow \infty}<0
$$

\section{Numerical Analysis, Case $N_{2}>0$}

Since the numerical analysis (for benchmark parametrization, see Table ??) allows for dispelling the ambiguity regarding the signs of several expressions:

$$
\begin{aligned}
& N_{1}<0, \quad N_{2}>0, \quad \Phi_{1}<0, \\
& \mu_{1}\left(\mu_{1}-r^{\star}\right) \omega_{3}^{1} N_{2}-\mu_{2}\left(\mu_{2}-r^{\star}\right) \omega_{3}^{2} N_{1}>0,
\end{aligned}
$$

we are able to indicate the signs of the slopes of demarcation lines and trajectories:

$$
\begin{aligned}
\left.\frac{\mathrm{d} b}{\mathrm{~d} k}\right|_{\dot{k}=0} & =\frac{\mu_{1}\left(\mu_{1}-r^{\star}\right) \omega_{3}^{1} N_{2}-\mu_{2}\left(\mu_{2}-r^{\star}\right) \omega_{3}^{2} N_{1}}{\left(\mu_{1}-r^{\star}\right)\left(\mu_{2}-r^{\star}\right) \omega_{3}^{1} \omega_{3}^{2}\left(\mu_{1}-\mu_{2}\right)}=\frac{(+)}{(-)}<0, \\
\left.\frac{\mathrm{d} b}{\mathrm{~d} k}\right|_{\dot{b}=0} & =\frac{N_{1} N_{2}\left(\mu_{1}-\mu_{2}\right)}{\mu_{1}\left(\mu_{2}-r^{\star}\right) \omega_{3}^{2} N_{1}-\mu_{2}\left(\mu_{1}-r^{\star}\right) \omega_{3}^{1} N_{2}}=\frac{(+)}{(-)}<0, \\
\frac{\partial \dot{b}}{\partial k} & =\frac{N_{1} N_{2}\left(\mu_{1}-\mu_{2}\right)}{\left(\mu_{1}-r^{\star}\right) \omega_{3}^{1} N_{2}-\left(\mu_{2}-r^{\star}\right) \omega_{3}^{2} N_{1}}=\frac{(+)}{(-)}<0, \\
\left.\left(\frac{\mathrm{d} b}{\mathrm{~d} k}\right)\right|_{t \rightarrow \infty} & =\frac{N_{2}}{\left(\mu_{2}-r^{\star}\right) \omega_{3}^{2}}=\frac{(+)}{(-)}<0 .
\end{aligned}
$$

Since the slopes of the demarcation lines $\dot{k}=0$ and $\dot{b}=0$ are both negative, we have to compare them:

$$
\begin{aligned}
\left.\frac{\mathrm{d} b}{\mathrm{~d} k}\right|_{\dot{k}=0}-\left.\frac{\mathrm{d} b}{\mathrm{~d} k}\right|_{\dot{b}=0} & =\frac{\left[\mu_{1}\left(\mu_{1}-r^{\star}\right) \omega_{3}^{1} N_{2}-\mu_{2}\left(\mu_{2}-r^{\star}\right) \omega_{3}^{2} N_{1}\right]\left(\mu_{1}-\mu_{2}\right) N_{1} N_{2}}{\mu_{1} \mu_{2}\left[\left(\mu_{1}-r^{\star}\right) \omega_{3}^{1} N_{2}-\left(\mu_{2}-r^{\star}\right) \omega_{3}^{2} N_{1}\right]^{2}} \\
& =\frac{(-)}{(+)}<0,
\end{aligned}
$$


$\mu_{1}\left(\mu_{1}-r^{\star}\right) \omega_{3}^{1} N_{2}-\mu_{2}\left(\mu_{2}-r^{\star}\right) \omega_{3}^{2} N_{1}>0$ if $N_{1}<0$ and $N_{2}>0$.

In addition, we have to compare the slope of the demarcation line $\dot{b}=0$ with the slope of the trajectory when the system approaches the steady-state:

$$
\begin{aligned}
\left.\left(\frac{\mathrm{d} b}{\mathrm{~d} k}\right)\right|_{t \rightarrow \infty}-\left.\frac{\mathrm{d} b}{\mathrm{~d} k}\right|_{\dot{b}=0} & =\frac{\mu_{2} N_{2}\left[\left(\mu_{2}-r^{\star}\right) \omega_{3}^{2} N_{1}-\left(\mu_{1}-r^{\star}\right) \omega_{3}^{1} N_{2}\right]}{\left(\mu_{2}-r^{\star}\right) \omega_{3}^{2}\left[\mu_{1}\left(\mu_{2}-r^{\star}\right) \omega_{3}^{2} N_{1}-\mu_{2}\left(\mu_{1}-r^{\star}\right) \omega_{3}^{1} N_{2}\right]} \\
& =\frac{(-)}{(+)}<0,
\end{aligned}
$$

where we referred to expressions (48) and (109b) to determine the sign of (117).

From (115a), (115b), and (115d) and (113), (116) and (117), we deduce the following inequalities:

$$
\left.\frac{\mathrm{d} b}{\mathrm{~d} k}\right|_{\dot{k}=0}<\left.\left(\frac{\mathrm{d} b}{\mathrm{~d} k}\right)\right|_{t \rightarrow \infty}<\left.\frac{\mathrm{d} b}{\mathrm{~d} k}\right|_{\dot{b}=0}<0
$$

\section{Transitional Dynamics for $b(t)$}

We turn now to the investigation of the transitional dynamics for the stock of international assets.

\section{Government Spending Shocks}

In a first place, we have to determine the initial reaction of the current account. At this end, we differentiate the two-dimensional stable solution for $b(t)$ w.r.t time, evaluate at $t=0$ and differentiate w.r.t $g^{j}$. with $i=d, f$ One obtains the initial response of the current account following an unanticipated permanent rise in government expenditure on the domestic good:

$$
\begin{aligned}
\frac{\mathrm{d} \dot{b}(0)}{\mathrm{d} g^{j}} & =\mu_{1} \frac{N_{1}}{\mu_{1}-r^{\star}} \frac{A_{1}}{\mathrm{~d} g^{j}}+\mu_{2} \frac{N_{2}}{\mu_{2}-r^{\star}} \frac{A_{2}}{\mathrm{~d} g^{j}} \quad i=d, f \\
& =-\left[\frac{\mu_{1}\left(\mu_{2}-r^{\star}\right) \omega_{3}^{2} N_{1}-\mu_{2}\left(\mu_{1}-r^{\star}\right) \omega_{3}^{1} N_{2}}{\left(\mu_{1}-r^{\star}\right)\left(\mu_{2}-r^{\star}\right)\left(\omega_{3}^{2}-\omega_{3}^{1}\right)}\right] \frac{\mathrm{d} \tilde{s}}{\mathrm{~d} g^{j}}, \quad i=d, f \\
& =-\frac{(-)}{(+)} \times(-)<0,
\end{aligned}
$$

where we have substituted the expressions of constants (see (75)); we used expression (109b) and that fact that $\left(\omega_{3}^{2}-\omega_{3}^{1}\right)>0$ (see (46)) to determine the sign of (119). From (119), the current account unambiguously deteriorates at time $t=0$.

Along the stable path, the current account evolves according to the following dynamic 
equation:

$$
\dot{b}(t)=\underbrace{\mu_{1} \frac{N_{1}}{\mu_{1}-r^{\star}} \frac{A_{1}}{\mathrm{~d} g^{j}} \mathrm{~d} g^{j}}_{(-)} e^{\mu_{1} t}+\underbrace{\mu_{2} \frac{N_{2}}{\mu_{2}-r^{\star}} \frac{A_{2}}{\mathrm{~d} g^{j}} \mathrm{~d} g^{j}}_{(?)} e^{\mu_{2} t}, \quad i=d, f,
$$

where $N_{1}<0$ (for all parametrization), $N_{2} \gtrless 0, \mu_{1}<\mu_{2}<0, A_{1} / \mathrm{d} g^{j}>0$, and $A_{2} / \mathrm{d} g^{j}<0$.

\section{D.3 Stable Manifold in the $(b, p)$-space}

The stable solution is given by the following system of equations:

$$
\begin{aligned}
p(t)-\tilde{p} & =\omega_{4}^{1} A_{1} e^{\mu_{1} t}+\omega_{4}^{2} A_{2} e^{\mu_{2} t}, \\
b(t)-\tilde{b} & =\frac{N_{1} A_{1}}{\mu_{1}-r^{\star}} e^{\mu_{1} t}+\frac{N_{2} A_{2}}{\mu_{2}-r^{\star}} e^{\mu_{2} t} .
\end{aligned}
$$

\section{Demarcation Lines}

Time derivatives of solutions (121a) and (121b) and elimination of $A_{1} e^{\mu_{1} t}$ and $A_{2} e^{\mu_{2} t}$ leads to a new linear differential equation system for $p(t)$ and $b(t)$ :

$$
\begin{aligned}
\dot{p}(t)= & \frac{\left(\mu_{1}-r^{\star}\right) \mu_{1} \omega_{4}^{1} N_{2}-\left(\mu_{2}-r^{\star}\right) \mu_{2} \omega_{4}^{2} N_{1}}{\left(\mu_{1}-r^{\star}\right)\left(\mu_{2}-r^{\star}\right) \Delta^{b}}(p(t)-\tilde{p}) \\
& -\frac{\omega_{4}^{1} \omega_{4}^{2}\left(\mu_{1}-\mu_{2}\right)}{\Delta^{b}}(b(t)-\tilde{b}), \\
\dot{b}(t)= & \frac{N_{1} N_{2}\left(\mu_{1}-\mu_{2}\right)}{\left(\mu_{1}-r^{\star}\right)\left(\mu_{2}-r^{\star}\right) \Delta^{b}}(p(t)-\tilde{p}) \\
& -\left[\frac{\mu_{1}\left(\mu_{2}-r^{\star}\right) \omega_{4}^{2} N_{1}-\mu_{2}\left(\mu_{1}-r^{\star}\right) \omega_{4}^{1} N_{2}}{\left(\mu_{1}-r^{\star}\right)\left(\mu_{2}-r^{\star}\right) \Delta^{b}}\right](b(t)-\tilde{b}),
\end{aligned}
$$

where

$$
\Delta^{b}=\frac{\left(\mu_{1}-r^{\star}\right) \omega_{4}^{1} N_{2}-\left(\mu_{2}-r^{\star}\right) \omega_{4}^{2} N_{1}}{\left(\mu_{1}-r^{\star}\right)\left(\mu_{2}-r^{\star}\right)}=\frac{(-)}{(+)}<0 .
$$

The new differential equation system has two stable roots $\mu_{1}<0$ and $\mu_{2}<0$ with $\mu_{1}<\mu_{2}<0$; hence the steady-state is a stable node in $(b, p)$-space.

From (122), we determine the slopes of demarcation lines:

$$
\begin{aligned}
\left.\frac{\mathrm{d} p}{\mathrm{~d} b}\right|_{\dot{p}=0} & =\frac{\left(\mu_{1}-r^{\star}\right)\left(\mu_{2}-r^{\star}\right) \omega_{4}^{1} \omega_{4}^{2}\left(\mu_{1}-\mu_{2}\right)}{\mu_{1}\left(\mu_{1}-r^{\star}\right) \omega_{4}^{1} N_{2}-\mu_{2}\left(\mu_{2}-r^{\star}\right) \omega_{4}^{2} N_{1}}=\frac{(-)}{(+)}<0, \\
\frac{\partial \dot{p}}{\partial b} & =-\frac{\left(\mu_{1}-r^{\star}\right)\left(\mu_{2}-r^{\star}\right) \omega_{4}^{1} \omega_{4}^{2}\left(\mu_{1}-\mu_{2}\right)}{\left(\mu_{1}-r^{\star}\right) \omega_{4}^{1} N_{2}-\left(\mu_{2}-r^{\star}\right) \omega_{4}^{2} N_{1}}=-\frac{(-)}{(-)}<0, \\
\left.\frac{\mathrm{d} p}{\mathrm{~d} b}\right|_{\dot{b}=0} & =\frac{\mu_{1}\left(\mu_{2}-r^{\star}\right) \omega_{4}^{2} N_{1}-\mu_{2}\left(\mu_{1}-r^{\star}\right) \omega_{4}^{1} N_{2}}{N_{1} N_{2}\left(\mu_{1}-\mu_{2}\right)}=\frac{(-)}{(?)} \lessgtr 0, \\
\frac{\partial \dot{b}}{\partial p} & =\frac{N_{1} N_{2}\left(\mu_{1}-\mu_{2}\right)}{\left(\mu_{1}-r^{\star}\right) \omega_{4}^{1} N_{2}-\left(\mu_{2}-r^{\star}\right) \omega_{4}^{2} N_{1}}=\frac{(?)}{(-)} \lessgtr 0 .
\end{aligned}
$$


We have computed several expressions as follows:

$$
\begin{aligned}
& \mu_{1}\left(\mu_{1}-r^{\star}\right) \omega_{4}^{1} N_{2}-\mu_{2}\left(\mu_{2}-r^{\star}\right) \omega_{4}^{2} N_{1} \\
= & \tilde{p} F_{k k}\left[\left(\mu_{1}-r^{\star}\right) \omega_{3}^{1} N_{2}-\left(\mu_{2}-r^{\star}\right) \omega_{3}^{2} N_{1}\right]>0, \\
& \left(\mu_{1}-r^{\star}\right) \omega_{4}^{1} N_{2}-\left(\mu_{2}-r^{\star}\right) \omega_{4}^{2} N_{1} \\
= & -\tilde{p} F_{k k} \frac{\left(\mu_{2}-r^{\star}\right) \mu_{1} \omega_{3}^{2} N_{1}-\left(\mu_{1}-r^{\star}\right) \mu_{2} \omega_{3}^{1} N_{2}}{\mu_{1} \mu_{2}}=(+) \times \frac{(-)}{(+)}<0, \\
& \mu_{1}\left(\mu_{2}-r^{\star}\right) \omega_{4}^{2} N_{1}-\mu_{2}\left(\mu_{1}-r^{\star}\right) \omega_{4}^{1} N_{2} \\
= & \frac{\omega_{4}^{1} \omega_{4}^{2}}{I_{c} \tilde{p} F_{k k}} \frac{p_{c}}{\tilde{p}}\left\{\mu_{3}\left[\tilde{p} F_{k k}\left(I_{p}+\left(1-\alpha_{c}\right) \frac{\tilde{X}}{\tilde{p}}\right)+\alpha_{c} \mu_{1} \mu_{4}\right]\right. \\
& \left.+\mu_{2}\left[\tilde{p} F_{k k}\left(I_{p}+\left(1-\alpha_{c}\right) \frac{\tilde{X}}{\tilde{p}}\right)+\alpha_{c} \mu_{3} \mu_{4}\right]\right\}=(+) \times[(-)+(-)]<0 .
\end{aligned}
$$

We used the fact that $\omega_{4}^{i}=\frac{\tilde{p} F_{k k}}{\mu_{i}} \omega_{3}^{i}>0$ to rewrite (125a) and (125b). The sign of (125b) follows from the following inequality $\left(\mu_{1}-r^{\star}\right) \omega_{3}^{1} N_{2}-\left(\mu_{2}-r^{\star}\right) \omega_{3}^{2} N_{1}<0$. The sign of (125b) follows from (109b). Regarding the sign of (125c), the first term in square brackets has an ambiguous sign. Since for all parametrization, numerical results indicate that $N_{1}<0$, we thus assume that the following inequality holds such that $\left[\tilde{p} F_{k k}\left(I_{p}+\left(1-\alpha_{c}\right) \frac{\tilde{X}}{\tilde{p}}\right)+\alpha_{c} \mu_{1} \mu_{4}\right]<0$.

\section{Fiscal Expansion and Trajectories}

The slope of the trajectory at time $t$ is obtained by taking the time derivative of the system of equations (121) and dividing the resulting expressions:

$$
\frac{\mathrm{d} p(t)}{\mathrm{d} b(t)}=\frac{\mu_{1} \omega_{4}^{1} A_{1} e^{\mu_{1} t}+\mu_{2} \omega_{4}^{2} A_{2} e^{\mu_{2} t}}{\mu_{1} \frac{N_{1} A_{1}}{\mu_{1}-r^{\star}} e^{\mu_{1} t}+\mu_{2} \frac{N_{2} A_{2}}{\mu_{2}-r^{\star}} e^{\mu_{2} t}}=\frac{\mu_{1} \omega_{4}^{1} A_{1} e^{\left(\mu_{1}-\mu_{2}\right) t}+\mu_{2} \omega_{4}^{2} A_{2}}{\mu_{1} \frac{N_{1} A_{1}}{\mu_{1}-r^{\star}} e^{\left(\mu_{1}-\mu_{2}\right) t}+\mu_{2} \frac{N_{2} A_{2}}{\mu_{2}-r^{\star}}} .
$$

The slope of the trajectory at the initial point can be calculated from (126) by setting $t=0$, and substituting the constants $A_{1}$ and $A_{2}$ given by (75):

$$
\left.\left(\frac{\mathrm{d} p}{\mathrm{~d} b}\right)\right|_{t \rightarrow 0}=\frac{\mu_{1} \omega_{4}^{1} \frac{A_{1}}{\mathrm{~d} g^{D}}+\mu_{2} \omega_{4}^{2} \frac{A_{2}}{\mathrm{~d} g^{D}}}{\mu_{1} \frac{N_{1}}{\mu_{1}-r^{\star}} \frac{A_{1}}{\mathrm{~d} g^{D}}+\mu_{2} \frac{N_{2}}{\mu_{2}-r^{\star}} \frac{A_{2}}{\mathrm{~d} g^{D}}}=0,
$$

where we have substituted the expressions of constants (see (75)) and used the fact that $\omega_{4}^{i}=$ $\frac{\tilde{p} F_{k k}}{\mu_{i}} \omega_{3}^{i}($ with $i=1,2)$.

Letting time tend towards infinity, i. e. $t \rightarrow \infty$, in equation (126), and keeping in mind that $\mu_{1}-\mu_{2}<0$, one obtains the slope of the trajectory approaching the steady-state:

$$
\left.\left(\frac{\mathrm{d} p}{\mathrm{~d} b}\right)\right|_{t \rightarrow \infty}=\frac{\left(\mu_{2}-r^{\star}\right) \omega_{4}^{2}}{N_{2}}=\frac{(-)}{(?)} \lessgtr 0 .
$$


We have to consider two cases, according to wether $N_{2} \gtrless 0$. Assuming that $N_{2}>0$, we get:

$$
\left.\frac{\mathrm{d} p}{\mathrm{~d} b}\right|_{\dot{b}=0}=\frac{(-)}{(+)}<0, \quad \frac{\partial \dot{b}}{\partial p}=\frac{(+)}{(-)}<0,\left.\quad\left(\frac{\mathrm{d} p}{\mathrm{~d} b}\right)\right|_{t \rightarrow \infty}=\frac{(-)}{(+)}<0
$$

Since the locus $\dot{p}=0$ and $\dot{b}=0$ are both negatively sloped, we have to estimate wether $\dot{p}=0$ is steeper or not than $\dot{b}=0$. Thus, we subtract (124c) from (124a):

$$
\begin{aligned}
\left.\frac{\mathrm{d} p}{\mathrm{~d} b}\right|_{\dot{p}=0}-\left.\frac{\mathrm{d} p}{\mathrm{~d} b}\right|_{\dot{b}=0} & =\frac{\mu_{1} \mu_{2}\left[\left(\mu_{1}-r^{\star}\right) \omega_{4}^{1} N_{2}-\left(\mu_{2}-r^{\star}\right) \omega_{4}^{2} N_{1}\right]^{2}}{\left[\mu_{1}\left(\mu_{1}-r^{\star}\right) \omega_{4}^{1} N_{2}-\mu_{2}\left(\mu_{2}-r^{\star}\right) \omega_{4}^{2} N_{1}\right]\left(\mu_{1}-\mu_{2}\right) N_{1} N_{2}} \\
& =\frac{(+)}{(+)}>0 .
\end{aligned}
$$

From (130), we can deduce that the locus $\dot{b}=0$ is steeper than the locus $\dot{p}=0$.

In addition, we have to compare the slope of the demarcation line $\dot{p}=0$ with the slope of the trajectory when the system approaches the steady-state:

$$
\begin{aligned}
\left.\left(\frac{\mathrm{d} p}{\mathrm{~d} b}\right)\right|_{t \rightarrow \infty}-\left.\frac{\mathrm{d} p}{\mathrm{~d} b}\right|_{\dot{p}=0} & =\frac{\mu_{2}\left(\mu_{2}-r^{\star}\right) \omega_{4}^{2}\left[\left(\mu_{1}-r^{\star}\right) \omega_{4}^{1} N_{2}-\left(\mu_{2}-r^{\star}\right) \omega_{4}^{2} N_{1}\right]}{N_{2}\left[\mu_{1}\left(\mu_{1}-r^{\star}\right) \omega_{4}^{1} N_{2}-\mu_{2}\left(\mu_{2}-r^{\star}\right) \omega_{4}^{2} N_{1}\right]} \\
& =\frac{(-)}{(+)}<0,
\end{aligned}
$$

where we referred to expressions (125a) and (125b) to determine the sign of (131).

From (124a), (124c), (128), (130), and (131), we deduce the following inequalities in the case $N_{2}>0$ :

$$
\left.\frac{\mathrm{d} p}{\mathrm{~d} b}\right|_{\dot{b}=0}<\left.\left(\frac{\mathrm{d} p}{\mathrm{~d} b}\right)\right|_{t \rightarrow \infty}<\left.\frac{\mathrm{d} p}{\mathrm{~d} b}\right|_{\dot{p}=0}<0 .
$$

Instead, by assuming that $N_{2}<0$, we get:

$$
\left.\frac{\mathrm{d} p}{\mathrm{~d} b}\right|_{\dot{b}=0}=\frac{(-)}{(-)}>0, \quad \frac{\partial \dot{b}}{\partial p}=\frac{(-)}{(-)}>0,\left.\quad\left(\frac{\mathrm{d} p}{\mathrm{~d} b}\right)\right|_{t \rightarrow \infty}=\frac{(-)}{(-)}>0
$$

In addition, we have to compare the (positive) slope of the demarcation line $\dot{b}=0$ with the (positive) slope of the trajectory when the system approaches the steady-state:

$$
\begin{aligned}
\left.\left(\frac{\mathrm{d} p}{\mathrm{~d} b}\right)\right|_{t \rightarrow \infty}-\left.\frac{\mathrm{d} p}{\mathrm{~d} b}\right|_{\dot{p}=0} & =-\frac{\mu_{2}\left[\left(\mu_{1}-r^{\star}\right) \omega_{4}^{1} N_{2}-\left(\mu_{2}-r^{\star}\right) \omega_{4}^{2} N_{1}\right]}{N_{1} N_{2}\left(\mu_{1}-\mu_{2}\right)} \\
& =-\frac{(+)}{(-)}>0,
\end{aligned}
$$

where the sign follows from equation (125b) and the fact that the case $N_{2}<0$ is considered. 
From (124a), (124c) and (134), we can deduce the following inequalities in the case $N_{2}<0$ :

$$
\left.\frac{\mathrm{d} p}{\mathrm{~d} b}\right|_{\dot{p}=0}<0<\left.\left(\frac{\mathrm{d} p}{\mathrm{~d} b}\right)\right|_{t \rightarrow \infty}<\left.\frac{\mathrm{d} p}{\mathrm{~d} b}\right|_{\dot{b}=0} .
$$

The Pattern of the Transitional Path in the (b,p)-space

To investigate in more details the conditions under which the stock of foreign bonds adjusts non monotonically, we have to determine wether there exists a critical value of time, denoted by $t=\tilde{t}>0$, such that the stock of traded bonds reaches a turning point along the stable trajectory, i. e. $c a(\tilde{t})=\dot{b}(\tilde{t})=0$. Setting (120) equal to zero and solving for $\tilde{t}$, we get:

$$
\tilde{t}=\frac{1}{\mu_{1}-\mu_{2}} \ln \left[-\frac{\mu_{2}\left(\mu_{1}-r^{\star}\right) N_{2} A_{2} / \mathrm{d} g^{j}}{\mu_{1}\left(\mu_{2}-r^{\star}\right) N_{1} A_{1} / \mathrm{d} g^{j}}\right]
$$

where $N_{1}<0$ for all parametrization and $N_{2} \gtrless 0$.

In the case $N_{2}>0$, the critical value of time along the transitional path does not exist since the term in square brackets is negative.

Unlike, in the case $N_{2}<0$, there exists a critical value of time, $t=\tilde{t}>0$, such that the stock of international assets reaches a turning point along the trajectory under the necessary condition:

$$
\mu_{1} \frac{N_{1}}{\mu_{1}-r^{\star}} \frac{A_{1}}{\mathrm{~d} g^{j}}+\mu_{2} \frac{N_{2}}{\mu_{2}-r^{\star}} \frac{A_{2}}{\mathrm{~d} g^{j}}<0 .
$$

In conclusion, regardless of the sign of $N_{2}$, the current account unambiguously deteriorates at time $t=0$. If $N_{2}<0$, the current account turns positive at time $\tilde{t}$ while the stock of international assets starts growing to its new lower steady-state level. Instead, if $N_{2}>0$, traded bonds holding falls monotonically and the current account stays in deficit while its size in absolute terms decreases.

\section{D.4 Stable Manifold in the $(s, c)$-space}

The stable solution is given by the following system of equations

$$
\begin{aligned}
& s(t)-\tilde{s}=A_{1} e^{\mu_{1} t}+A_{2} e^{\mu_{2} t}, \\
& c(t)-\tilde{c}=\omega_{2}^{1} A_{1} e^{\mu_{1} t}+\omega_{2}^{2} A_{2} e^{\mu_{2} t} .
\end{aligned}
$$




\section{Demarcation Lines}

Time derivatives of solutions (138a) and (138b) and elimination of $A_{1} e^{\mu_{1} t}$ and $A_{2} e^{\mu_{2} t}$ leads to a new linear differential equation system for $s(t)$ and $c(t)$

$$
\begin{aligned}
& \dot{s}(t)=\left[\frac{\mu_{1} \omega_{2}^{2}-\mu_{2} \omega_{2}^{1}}{\Delta^{c}}\right](s(t)-\tilde{s})-\left[\frac{\mu_{1}-\mu_{2}}{\Delta^{c}}\right](c(t)-\tilde{c}), \\
& \dot{c}(t)=\left[\frac{\omega_{2}^{1} \omega_{2}^{2}\left(\mu_{1}-\mu_{2}\right)}{\Delta^{c}}\right](s(t)-\tilde{s})-\left[\frac{\mu_{1} \omega_{2}^{1}-\mu_{2} \omega_{2}^{2}}{\Delta^{c}}\right](c(t)-\tilde{c}),
\end{aligned}
$$

with

$$
\Delta^{c}=\frac{\mu_{2}-\mu_{1}}{\sigma}>0
$$

The new differential equation system has two stable roots $\mu_{1}<0$ and $\mu_{2}<0$ with $\mu_{1}<\mu_{2}<0$ (see $(23))$; hence the steady-state is a stable node in $(s, c)$-space.

From (139), we determine the slopes of demarcation lines:

$$
\begin{aligned}
\left.\frac{\mathrm{d} c}{\mathrm{~d} s}\right|_{\dot{s}=0} & =\frac{\mu_{1} \omega_{2}^{2}-\mu_{2} \omega_{2}^{1}}{\mu_{1}-\mu_{2}}=1, \\
\frac{\partial \dot{s}}{\partial c} & =\sigma>0, \\
\left.\frac{\mathrm{d} c}{\mathrm{~d} s}\right|_{\dot{c}=0} & =\frac{\omega_{2}^{1} \omega_{2}^{2}\left(\mu_{1}-\mu_{2}\right)}{\mu_{1} \omega_{2}^{1}-\mu_{2} \omega_{2}^{2}}=\frac{\left(\sigma+\mu_{1}\right)\left(\sigma+\mu_{2}\right)}{\sigma\left[\sigma+\left(\mu_{1}+\mu_{2}\right)\right]} \\
& =\frac{\sigma\left[\sigma+\left(\mu_{1}+\mu_{2}\right)\right]+\mu_{1} \mu_{2}}{\sigma\left[\sigma+\left(\mu_{1}+\mu_{2}\right)\right]}=1+\frac{\mu_{1} \mu_{2}}{\sigma\left[\sigma+\left(\mu_{1}+\mu_{2}\right)\right]}=\frac{(+)}{(?)}, \\
\frac{\partial \dot{c}}{\partial s} & =-\frac{\left(\sigma+\mu_{1}\right)\left(\sigma+\mu_{2}\right)}{\sigma}<0,
\end{aligned}
$$

where the sign of the slope of the demarcation line $\dot{c}=0$ remains undetermined. More specifically, its sign depends crucially upon the degree of habit persistence in consumption, reflected by $\left|\mu_{1}\right|$, and the price-elasticity of export demand, $\nu_{X}$. For plausible values of parameters for preferences, production function, and export function, we find that $\sigma+\left(\mu_{1}+\mu_{2}\right)>0$ and we will assume that this inequality holds from thereon.

Since the demarcation lines $\dot{c}=0$ and $\dot{s}=0$ have positive slopes, we have to compare their magnitudes:

$$
\left.\frac{\mathrm{d} c}{\mathrm{~d} s}\right|_{\dot{s}=0}-\left.\frac{\mathrm{d} c}{\mathrm{~d} s}\right|_{\dot{c}=0}=-\frac{\mu_{1} \mu_{2}}{\sigma\left[\sigma+\left(\mu_{1}+\mu_{2}\right)\right]}<0
$$

from which we deduce the following inequality:

$$
0<\left.\frac{\mathrm{d} c}{\mathrm{~d} s}\right|_{\dot{s}=0}=1<\left.\frac{\mathrm{d} c}{\mathrm{~d} s}\right|_{\dot{c}=0}
$$


The slope of the trajectory at time $t$ is obtained by differentiating the system of equations (138) with respect to time $t$ and dividing the resulting expressions:

$$
\frac{\mathrm{d} c(t)}{\mathrm{d} s(t)}=\frac{\mu_{1} \omega_{2}^{1} A_{1} e^{\mu_{1} t}+\mu_{2} \omega_{2}^{2} A_{2} e^{\mu_{2} t}}{\mu_{1} A_{1} e^{\mu_{1} t}+\mu_{2} A_{2} e^{\mu_{2} t}}=\frac{\mu_{1} \omega_{2}^{1} A_{1} e^{\left(\mu_{1}-\mu_{2}\right) t}+\mu_{2} \omega_{2}^{2} A_{2}}{\mu_{1} A_{1} e^{\left(\mu_{1}-\mu_{2}\right) t}+\mu_{2} A_{2}} .
$$

The slope of the trajectory at the initial point can be calculated from (144) by setting $t=0$, and substituting the constants $A_{1}$ and $A_{2}$ given by (75)

$$
\begin{aligned}
\left.\left(\frac{\mathrm{d} c}{\mathrm{~d} s}\right)\right|_{t \rightarrow 0} & =\frac{\mu_{1} \omega_{2}^{1} \frac{A_{1}}{\mathrm{~d} g^{D}}+\mu_{2} \omega_{2}^{2} \frac{A_{2}}{\mathrm{~d} g^{D}}}{\mu_{1} \frac{A_{1}}{\mathrm{~d} g^{D}}+\mu_{2} \frac{A_{2}}{\mathrm{~d} g^{D}}} \\
& =\left[\frac{\mu_{1} \omega_{2}^{1} \omega_{3}^{2}-\mu_{2} \omega_{2}^{2} \omega_{3}^{1}}{\mu_{1} \omega_{3}^{2}-\mu_{2} \omega_{3}^{1}}\right]=\frac{(-)}{(-)}>0,
\end{aligned}
$$

where expressions in the numerator and the denominator of (145) write as follows:

$$
\begin{aligned}
& \mu_{1} \omega_{2}^{1} \omega_{3}^{2}-\mu_{2} \omega_{2}^{2} \omega_{3}^{1} \\
= & -\frac{I_{c} \mu_{1} \mu_{2}\left(\mu_{1}-\mu_{2}\right)\left(\sigma+\mu_{1}\right)\left(\sigma+\mu_{2}\right)\left[r^{\star}-\left(\mu_{1}+\mu_{2}\right)\right]}{\sigma^{2}\left[\mu_{1} \mu_{4}+I_{p} \tilde{p} F_{k k}\right]\left[\mu_{2} \mu_{3}+I_{p} \tilde{p} F_{k k}\right]}=-\frac{(+)}{(+)}<0, \\
& \mu_{1} \omega_{3}^{2}-\mu_{2} \omega_{3}^{1} \\
= & -\frac{I_{c} \mu_{1} \mu_{2}\left(\mu_{2}-\mu_{1}\right)\left\{\left[\mu_{1} \mu_{4}+I_{p} \tilde{p} F_{k k}\right]-\left(\sigma+\mu_{1}\right)\left(\mu_{4}-\mu_{2}\right)\right\}}{\sigma\left[\mu_{1} \mu_{4}+I_{p} \tilde{p} F_{k k}\right]\left[\mu_{2} \mu_{3}+I_{p} \tilde{p} F_{k k}\right]}=-\frac{(+)}{(+)}<0,
\end{aligned}
$$

where we used the fact that $\mu_{1} \mu_{4}-\mu_{2} \mu_{3}=\left(\mu_{1}-\mu_{2}\right)\left[r^{\star}-\left(\mu_{1}+\mu_{2}\right)\right]$ and

$$
I_{p} \tilde{p} F_{k k}+\mu_{1} \mu_{2}-\sigma\left[r^{\star}-\left(\mu_{1}+\mu_{2}\right)\right]=\left[\mu_{1} \mu_{4}+I_{p} \tilde{p} F_{k k}\right]-\left(\sigma+\mu_{1}\right)\left(\mu_{4}-\mu_{2}\right)<0 .
$$

Letting time tend towards infinity, i. e. $t \rightarrow \infty$, in equation (144), one obtains the slope of the trajectory approaching the steady-state:

$$
\left.\left(\frac{\mathrm{d} c}{\mathrm{~d} s}\right)\right|_{t \rightarrow \infty}=\omega_{2}^{2}=\left(\frac{\sigma+\mu_{2}}{\sigma}\right)>0
$$

with $0<\left(\frac{\sigma+\mu_{2}}{\sigma}\right)<1$. Thus we can infer the following inequality:

$$
0<\left.\left(\frac{\mathrm{d} c}{\mathrm{~d} s}\right)\right|_{t \rightarrow \infty}<\left.\left(\frac{\mathrm{d} c}{\mathrm{~d} s}\right)\right|_{\dot{s}=0} .
$$

We compare the slope of the trajectory at the initial point with the slope of the trajectory approaching the steady-state:

$$
\left.\left(\frac{\mathrm{d} c}{\mathrm{~d} s}\right)\right|_{t \rightarrow 0}-\left.\left(\frac{\mathrm{d} c}{\mathrm{~d} s}\right)\right|_{t \rightarrow \infty}=\frac{\mu_{1}\left(\mu_{1}-\mu_{2}\right) \omega_{3}^{2}}{\mu_{1} \omega_{3}^{2}-\mu_{2} \omega_{3}^{1}}=\frac{(+)}{(-)}<0
$$


$\mu_{1} \omega_{3}^{2}-\mu_{2} \omega_{3}^{1}<0$ is given by (146b). From (149), we can deduce the following inequality:

$$
0<\left.\left(\frac{\mathrm{d} c}{\mathrm{~d} s}\right)\right|_{t \rightarrow 0}<\left.\left(\frac{\mathrm{d} c}{\mathrm{~d} s}\right)\right|_{t \rightarrow \infty}
$$

Finally, we compare the slope of the trajectory at the initial point with the slope of the demarcation line $\dot{s}=0$ :

$$
\left.\left(\frac{\mathrm{d} c}{\mathrm{~d} s}\right)\right|_{t \rightarrow 0}-\left.\left(\frac{\mathrm{d} c}{\mathrm{~d} s}\right)\right|_{\dot{s}=0}=\frac{\left(\mu_{1}\right)^{2} \omega_{3}^{2}-\left(\mu_{2}\right)^{2} \omega_{3}^{1}}{\sigma\left(\mu_{1} \omega_{3}^{2}-\mu_{2} \omega_{3}^{1}\right)}=\frac{(+)}{(-)}<0
$$

$\left(\mu_{1}\right)^{2} \omega_{3}^{2}-\left(\mu_{2}\right)^{2} \omega_{3}^{1}>0$ and $\mu_{1} \omega_{3}^{2}-\mu_{2} \omega_{3}^{1}<0$.

From (143), (149) and (151), we deduce the following inequality:

$$
0<\left.\left(\frac{\mathrm{d} c}{\mathrm{~d} s}\right)\right|_{t \rightarrow 0}<\left.\left(\frac{\mathrm{d} c}{\mathrm{~d} s}\right)\right|_{t \rightarrow \infty}<\left.\frac{\mathrm{d} c}{\mathrm{~d} s}\right|_{\dot{s}=0}=1<\left.\frac{\mathrm{d} c}{\mathrm{~d} s}\right|_{\dot{c}=0}
$$

Transitional Dynamics for $s(t)$ and $c(t)$

We turn now to the investigation of the transitional dynamics for the stock of habits and the real consumption.

\section{Government Spending Shocks}

The stable solution for $c(t)$ is given by:

$$
c(t)=\tilde{c}+\left(\frac{\sigma+\mu_{1}}{\sigma}\right) A_{1} e^{\mu_{1} t}+\left(\frac{\sigma+\mu_{2}}{\sigma}\right) A_{2} e^{\mu_{2} t}
$$

where we have substituted the expressions of eigenvectors $\omega_{2}^{1}$ and $\omega_{2}^{2}$. Setting $t=0$, differentiating with respect to $g^{j}$ (with $i=d, f$ ), and substituting the constants given by (75), we obtain the initial response of real consumption following an unanticipated permanent rise in government spending:

$$
\begin{aligned}
\frac{\mathrm{d} c(0)}{\mathrm{d} g^{j}} & =\frac{\mathrm{d} \tilde{c}}{\mathrm{~d} g^{j}}+\left(\frac{\sigma+\mu_{1}}{\sigma}\right) \frac{A_{1}}{\mathrm{~d} g^{j}}+\left(\frac{\sigma+\mu_{2}}{\sigma}\right) \frac{A_{2}}{\mathrm{~d} g^{j}} \\
& =-\left[\frac{\mu_{1} \omega_{3}^{2}-\mu_{2} \omega_{3}^{1}}{\sigma\left(\omega_{3}^{2}-\omega_{3}^{1}\right)}\right] \frac{\mathrm{d} \tilde{c}}{\mathrm{~d} g^{j}}=-\frac{(-)}{(+)} \times(-)<0, \quad i=d, f
\end{aligned}
$$

where $\omega_{3}^{2}-\omega_{3}^{1}>0$ and $\mu_{1} \omega_{3}^{2}-\mu_{2} \omega_{3}^{1}<0$ is given by $(146 \mathrm{~b})$.

Equation (153) indicates that real consumption falls initially as in the long-run. In addition, we have to evaluate the magnitude of the initial change w. r. t. the long-run variation. At this end, we determinate if the numerator is lower or higher than the denominator. Using (46) and 
(146b), we can rewrite the term in square brackets, appearing in expression (153) of the initial change of the real consumption, as follows:

$$
\begin{aligned}
& -\left[\frac{\mu_{1} \omega_{3}^{2}-\mu_{2} \omega_{3}^{1}}{\sigma\left(\omega_{3}^{2}-\omega_{3}^{1}\right)}\right] \\
= & -\frac{\mu_{1} \mu_{2}\left\{\left[\mu_{1} \mu_{4}+I_{p} \tilde{p} F_{k k}\right]-\left(\sigma+\mu_{1}\right)\left(\mu_{4}-\mu_{2}\right)\right\}}{\sigma\left\{\mu_{1} \mu_{2}\left(\sigma+r^{\star}\right)+I_{p} \tilde{p} F_{k k}\left[\sigma+\left(\mu_{1}+\mu_{2}\right)\right]\right\}} \\
= & \frac{\mu_{1} \mu_{2}\left\{\left[\mu_{1} \mu_{4}+I_{p} \tilde{p} F_{k k}\right]-\left(\sigma+\mu_{1}\right)\left(\mu_{4}-\mu_{2}\right)\right\}}{\mu_{1} \mu_{2}\left\{\left[\mu_{1} \mu_{4}+I_{p} \tilde{p} F_{k k}\right]-\left(\sigma+\mu_{1}\right)\left(\sigma+\mu_{4}\right)\right\}-\left(\sigma+\mu_{1}\right)\left(\sigma+\mu_{2}\right) I_{p} \tilde{p} F_{k k}}, \\
= & \frac{(-)}{(-)}>0,
\end{aligned}
$$

where $I_{c}$ and $\left(\mu_{2}-\mu_{1}\right)$ cancel to obtain the first line and we have rewritten the term in square brackets located in the denominator as follows:

$$
\begin{aligned}
& \mu_{1} \mu_{2} \sigma\left(\sigma+r^{\star}\right)+I_{p} \tilde{p} F_{k k} \sigma\left[\sigma+\left(\mu_{1}+\mu_{2}\right)\right] \\
= & \left(\sigma+\mu_{1}\right)\left[\mu_{1} \mu_{2}\left(\sigma+\mu_{4}\right)+\left(\sigma+\mu_{2}\right) I_{p} \tilde{p} F_{k k}\right]-\mu_{1} \mu_{2}\left[\mu_{1} \mu_{4}+I_{p} \tilde{p} F_{k k}\right]>0,
\end{aligned}
$$

where we used the fact that $\sigma\left(\sigma+r^{\star}\right)=\left(\sigma+\mu_{1}\right)\left(\sigma+\mu_{4}\right)-\mu_{1} \mu_{4}$ and $\sigma\left[\sigma+\left(\mu_{1}+\mu_{2}\right)\right]=$ $\left(\sigma+\mu_{1}\right)\left(\sigma+\mu_{2}\right)-\mu_{1} \mu_{2}$

Because $\left(\sigma+\mu_{4}\right)>\left(\mu_{4}-\mu_{2}\right)$ (since $\left.\left(\sigma+\mu_{2}\right)>0\right)$, the first term in square brackets in the denominator of (154) is higher than numerator. Therefore, real consumption decreases instantaneously after a permanent fiscal expansion, but by a lower amount than in the longrun. This behavior is due to the existence of habit persistence in consumption which makes agents reluctant to reduce strongly their real consumption and incites them to decumulate financial wealth in order to maintain their habitual standard of living. We can summarize our discussion with the following inequality:

$$
\frac{\mathrm{d} \tilde{c}}{\mathrm{~d} g^{j}}<\frac{\mathrm{d} c(0)}{\mathrm{d} g^{j}}<0, \quad i=d, f .
$$

Differentiate the stable solution for $s(t)$ and for $c(t)$ w.r.t. time, we get the temporal paths followed by habits and real consumption:

$$
\begin{aligned}
& \dot{s}(t)=\underbrace{\mu_{1} \frac{A_{1}}{\mathrm{~d} g^{j}} \mathrm{~d} g^{j}}_{(-)} e^{\mu_{1} t}+\underbrace{\mu_{2} \frac{A_{2}}{\mathrm{~d} g^{j}} \mathrm{~d} g^{j}}_{(+)} e^{\mu_{2} t}, \quad i=d, f, \\
& \dot{c}(t)=\underbrace{\mu_{1}\left(\frac{\sigma+\mu_{1}}{\sigma}\right) \frac{A_{1}}{\mathrm{~d} g^{j}} \mathrm{~d} g^{j}}_{(-)} e^{\mu_{1} t}+\underbrace{\mu_{2}\left(\frac{\sigma+\mu_{2}}{\sigma}\right) \frac{A_{2}}{\mathrm{~d} g^{j}} \mathrm{~d} g^{j}}_{(+)} e^{\mu_{2} t}, \quad i=d, f,
\end{aligned}
$$


where $A_{1} / \mathrm{d} g^{j}>0$ and $A_{2} / \mathrm{d} g^{j}<0$ (see (75)). At a first sight, from (157), the transitional paths for $c(t)$ and $s(t)$ may be monotonic or non-monotonic.

Evaluate (157a) and (158c) at time $t=0$, we obtain the initial changes of $s$ and $c$ once real consumption jumped:

$$
\begin{aligned}
\frac{\mathrm{d} \dot{s}(0)}{\mathrm{d} g^{j}} & =\underbrace{\mu_{1} \frac{A_{1}}{\mathrm{~d} g^{j}}}_{(-)}+\underbrace{\mu_{2} \frac{A_{2}}{\mathrm{~d} g^{j}}}_{(+)}, \\
& =-\left[\frac{\mu_{1} \omega_{3}^{2}-\mu_{2} \omega_{3}^{1}}{\omega_{3}^{2}-\omega_{3}^{1}}\right] \frac{\mathrm{d} \tilde{s}}{\mathrm{~d} g^{j}}=-\frac{(-)}{(+)} \times(-)<0, \\
\frac{\mathrm{d} \dot{c}(0)}{\mathrm{d} g^{j}} & =\underbrace{\mu_{1}\left(\frac{\sigma+\mu_{1}}{\sigma}\right) \frac{A_{1}}{\mathrm{~d} g^{j}}}_{(-)}+\underbrace{\mu_{2}\left(\frac{\sigma+\mu_{2}}{\sigma}\right) \frac{A_{2}}{\mathrm{~d} g^{j}}}_{(+)}, \\
& =-\left[\frac{\mu_{1}\left(\sigma+\mu_{1}\right) \omega_{3}^{2}-\mu_{2}\left(\sigma+\mu_{2}\right) \omega_{3}^{1}}{\sigma\left(\omega_{3}^{2}-\omega_{3}^{1}\right)}\right] \frac{\mathrm{d} \tilde{c}}{\mathrm{~d} g^{j}}=-\frac{(-)}{(+)} \times(-)<0,
\end{aligned}
$$

with $\left(\omega_{3}^{2}-\omega_{3}^{1}\right)>0$, and

$$
\begin{aligned}
& \mu_{1}\left(\sigma+\mu_{1}\right) \omega_{3}^{2}-\mu_{2}\left(\sigma+\mu_{2}\right) \omega_{3}^{1} \\
= & -\frac{I_{c} \mu_{1} \mu_{2}\left(\sigma+\mu_{1}\right)\left(\sigma+\mu_{2}\right)\left(\mu_{1}-\mu_{2}\right)\left[r^{\star}-\left(\mu_{1}+\mu_{2}\right)\right]}{\sigma\left[\mu_{1} \mu_{4}+I_{p} \tilde{p} F_{k k}\right]\left[\mu_{2} \mu_{3}+I_{p} \tilde{p} F_{k k}\right]}=-\frac{(+)}{(+)}<0,
\end{aligned}
$$

where we used the fact that $\mu_{1} \mu_{4}-\mu_{2} \mu_{3}=\left[r^{\star}-\left(\mu_{1}+\mu_{2}\right)\right]>0$.

We investigate in more details the condition of the non-monotonic adjustment of the reference stock and the real consumption. Adopting the same procedure than previously, there exists a critical value of time, $t=t>0$, such that the habit stock reaches a turning point, i. e. $\dot{s}(\hat{t})=\dot{s}(\hat{t})=0$. Solving the first line of (98a), we obtain:

$$
\dot{t}=\frac{1}{\mu_{1}-\mu_{2}} \ln \left[-\frac{\mu_{2} A_{2} / \mathrm{d} g^{j}}{\mu_{1} A_{1} / \mathrm{d} g^{j}}\right], \quad i=d, f
$$

where $\mu_{1}-\mu_{2}<0$ and the necessary condition for $t>0$ corresponds to:

$$
0<-\frac{\mu_{2} A_{2} / \mathrm{d} g^{j}}{\mu_{1} A_{1} / \mathrm{d} g^{j}}<1 \quad \Leftrightarrow \quad \frac{\mathrm{d} \dot{s}(0)}{\mathrm{d} g^{j}}<0 . \quad i=d, f .
$$

If the condition (161) holds, the stock of habits initially decreases before reaching a turning point at time $\dot{t}$. Subsequently, accumulation of habits turns to be positive and the reference stock increases towards its new lower steady-state level, i. e. $s$ overshoots its long-term value. 
Regarding real consumption's transitional path, there exists a critical value of time, $t=$ $\breve{t}>0$, such that the real consumption reaches a turning point, i. e. $\dot{c}(\breve{t})=0$. Solving the first line of (98a), we obtain:

$$
\breve{t}=\frac{1}{\mu_{1}-\mu_{2}} \ln \left[-\frac{\mu_{2} \omega_{2}^{2} A_{2} / \mathrm{d} g^{j}}{\mu_{1} \omega_{2}^{1} A_{1} / \mathrm{d} g^{j}}\right], \quad i=d, f
$$

where $\mu_{1}-\mu_{2}<0$ and the necessary condition for $\breve{t}>0$ corresponds to:

$$
0<-\frac{\mu_{2} \omega_{2}^{2} A_{2} / \mathrm{d} g^{j}}{\mu_{1} \omega_{2}^{1} A_{1} / \mathrm{d} g^{j}}<1 \quad \Leftrightarrow \quad \frac{\mathrm{d} \dot{c}(0)}{\mathrm{d} g^{j}}<0 . \quad i=d, f
$$

If the condition (163) holds, the real consumption initially decreases before reaching a turning point at time $\breve{t}$. Subsequently, the real consumption rises towards its new lower long-run level.

Finally, because $\mu_{1}<\mu_{2}<0$, and $\left(\sigma+\mu_{1}\right)>0,\left(\sigma+\mu_{2}\right)>0$, we have $0<\omega_{2}^{1}<\omega_{2}^{2}$ which implies in turn that $\frac{\mu_{2} \omega_{2}^{2} A_{2} / \mathrm{d} g^{j}}{\mu_{1} \omega_{2}^{1} A_{1} / \mathrm{d} g^{j}}>\frac{\mu_{2} A_{2} / \mathrm{d} g^{j}}{\mu_{1} A_{1} / \mathrm{d} g^{j}}$. Thus, we deduce the following inequality:

$$
0<\breve{t}<\dot{t}
$$

In words, the trajectory cuts first the demarcation line $\dot{c}=0$ at time $\breve{t}$, and then cuts the demarcation line $\dot{s}=0$ at time $\dot{t}$ and finally the dynamic system approaches the steady-state.

\section{D.5 Non-monotonic Transitional Paths and the Range of Dates}

In this section, we determine analytically the range of dates, i. e. the time at which the dynamics of key economic variables change of direction as they follow non-monotonic transitional paths. More specifically, we have four dates, say respectively $\hat{t}, \breve{t}, t$ and $\tilde{t}$ at which investment, real consumption, accumulation of consumption experience and current account change of sign. The range of dates is not a crucial task but rather allows for a more rigorous discussion of transitional paths. We have already shown that $\breve{t}<\dot{t}$ (see (164)). We have now to compare $\hat{t}$ with $t$ and $\breve{t}$ by making use of their expressions given respectively by (91), (160) and (162).

For the inequality $\hat{t}<t$ to hold, the following condition must be satisfied

$$
0<-\frac{\mu_{2} A_{2} / \mathrm{d} g^{j}}{\mu_{1} A_{1} / \mathrm{d} g^{j}}<-\frac{\mu_{2} \omega_{3}^{2} A_{2} / \mathrm{d} g^{j}}{\mu_{1} \omega_{3}^{1} A_{1} / \mathrm{d} g^{j}}<1 .
$$


It is straightforward to show that this necessary condition is respected if $\omega_{3}^{2}-\omega_{3}^{1}>0$. From (46), the latter inequality holds such that $\hat{t}<\dot{t}$.

For the inequality $\hat{t}<\breve{t}$ to hold, the following condition must be satisfied:

$$
0<-\frac{\mu_{2} \omega_{2}^{2} A_{2} / \mathrm{d} g^{j}}{\mu_{1} \omega_{2}^{1} A_{1} / \mathrm{d} g^{j}}<-\frac{\mu_{2} \omega_{3}^{2} A_{2} / \mathrm{d} g^{j}}{\mu_{1} \omega_{3}^{1} A_{1} / \mathrm{d} g^{j}}<1
$$

It is straightforward to show that this necessary condition is respected if $\omega_{2}^{1} \omega_{3}^{2}-\omega_{2}^{2} \omega_{3}^{1}>0$. We have computed the following expression to determine its sign:

$$
\begin{aligned}
& \omega_{2}^{1} \omega_{3}^{2}-\omega_{2}^{2} \omega_{3}^{1} \\
= & \frac{I_{c}\left(\sigma+\mu_{1}\right)\left(\sigma+\mu_{2}\right)\left(\mu_{1}-\mu_{2}\right)\left[\mu_{1} \mu_{2}+I_{p} \tilde{p} F_{k k}\right]}{\sigma^{2}\left[\mu_{1} \mu_{4}+I_{p} \tilde{p} F_{k k}\right]\left[\mu_{2} \mu_{3}+I_{p} \tilde{p} F_{k k}\right]}=\frac{(+)}{(+)}>0 .
\end{aligned}
$$

Since inequality (166) holds, we can deduce the following time range for turning points:

$$
0<\hat{t}<\breve{t}<\dot{t}<\tilde{t}
$$

From (168), we can deduce that investment in physical capital changes of sign before real consumption which itself changes of sign before accumulation of habits.

\section{D.6 Dynamics for the Rate of Time Preference}

As have underlined previously, the specification of a habit-forming behavior implies a variable time preference rate. Following an unanticipated permanent fiscal expansion, the time preference rate rises initially and then decreases over time toward its steady-state value, $\delta$. The reaction of $\rho$ reflects the temporary gap between the marginal utility of current real expense and the marginal utility of future real expense. In this section, we provide an analytical treatment of the adjustment of the time preference rate following a permanent fiscal expansion.

The solution for the time preference rate is obtained by linearizing (??) in the neighborhood of the steady-state and using the fact that $\tilde{\rho}=\delta$ :

$$
\begin{aligned}
\rho(t) & =\delta+\frac{\tilde{p}}{p_{c} \bar{\lambda}}(\delta+\sigma) u_{c c}(c(t)-\tilde{c})+\frac{\tilde{p}}{p_{c} \bar{\lambda}}(\delta+2 \sigma) \tilde{\Gamma}(s(t)-\tilde{s})+\frac{\tilde{p}}{p_{c} \bar{\lambda}}(\delta+\sigma) \frac{p_{c} \bar{\lambda}\left(1-\alpha_{c}\right)}{\tilde{p}^{2}}(p(t)-\tilde{p}), \\
& =\delta+\frac{\tilde{p}}{p_{c} \bar{\lambda}}(\delta+\sigma) u_{c c}\left[\Xi_{1} A_{1} e^{\mu_{1} t}+\Xi_{2} A_{2} e^{\mu_{2} t}\right],
\end{aligned}
$$


where

$$
\begin{aligned}
& \Xi_{1}=\omega_{2}^{1}+\frac{(\delta+2 \sigma) \tilde{\Gamma}}{(\delta+\sigma) \tilde{u}_{11}}+\frac{p_{c} \bar{\lambda}}{\tilde{p} \tilde{u}_{11}} \frac{\left(1-\alpha_{c}\right)}{\tilde{p}} \omega_{4}^{1}=-\frac{(\delta+2 \sigma) \tilde{\Gamma}}{(\delta+\sigma) \tilde{u}_{11}} \frac{\mu_{1}}{\left(\delta+\sigma-\mu_{1}\right)}<0, \\
& \Xi_{2}=\omega_{2}^{2}+\frac{(\delta+2 \sigma) \tilde{\Gamma}}{(\delta+\sigma) \tilde{u}_{11}}+\frac{p_{c} \bar{\lambda}}{\tilde{p} \tilde{u}_{11}} \frac{\left(1-\alpha_{c}\right)}{\tilde{p}} \omega_{4}^{2}=-\frac{(\delta+2 \sigma) \tilde{\Gamma}}{(\delta+\sigma) \tilde{u}_{11}} \frac{\mu_{2}}{\left(\delta+\sigma-\mu_{2}\right)}<0,
\end{aligned}
$$

with $\Gamma>0$. To determine the signs of $\Xi_{1}$ and $\Xi_{2}$, we made use of the second line of $\left(J-\mu_{i} I_{4 \times 4}\right) \omega_{j}^{i}=0$.

We estimate the initial reaction of the time preference rate by evaluating (169) at time $t=0$ and by differentiating w. r. t. $g^{j}$ :

$$
\begin{aligned}
\frac{\mathrm{d} \rho(0)}{\mathrm{d} g^{j}} & =\frac{\tilde{p}}{p_{c} \bar{\lambda}}(\delta+\sigma) u_{c c}\left[\Xi_{1} \frac{A_{1}}{\mathrm{~d} g^{j}}+\Xi_{2} \frac{A_{2}}{\mathrm{~d} g^{j}}\right], \\
& =-\frac{\tilde{p}}{p_{c} \bar{\lambda}} \frac{(\delta+2 \sigma) \tilde{\Gamma}}{\left(\delta+\sigma-\mu_{1}\right)\left(\delta+\sigma-\mu_{2}\right)\left(\omega_{3}^{2}-\omega_{3}^{1}\right)} \\
& \times\left[\mu_{1} \mu_{2}\left(\omega_{3}^{2}-\omega_{3}^{1}\right)-(\delta+\sigma)\left(\mu_{1} \omega_{3}^{2}-\mu_{2} \omega_{3}^{1}\right)\right] \frac{\mathrm{d} \tilde{s}}{\mathrm{~d} g^{j}}>0,
\end{aligned}
$$

where $\omega_{3}^{2}-\omega_{3}^{1}>0$ (see (46)) and $\mu_{1} \omega_{3}^{2}-\mu_{2} \omega_{3}^{1}<0$ see (146b)). We have substituted the expressions of constants given by (75) and made use of (170) to determine (171). From (171), the time preference rate rises initially giving rise to a decreasing temporal path for real consumption (see (??)).

We estimate now the initial change of the time preference rate by differentiating (169) w. r. t. time $t$, evaluating this at time $t=0$, and differentiating w. r. t. $g^{j}$

$$
\begin{aligned}
\frac{\mathrm{d} \dot{\rho}^{c}(0)}{\mathrm{d} g^{j}} & =\frac{\tilde{p}}{p_{c} \bar{\lambda}}(\delta+\sigma) u_{c c}\left[\mu_{1} \Xi_{1} \frac{A_{1}}{\mathrm{~d} g^{j}}+\mu_{2} \Xi_{2} \frac{A_{2}}{\mathrm{~d} g^{j}}\right], \\
& =-\frac{\tilde{p}}{p_{c} \bar{\lambda}} \frac{(\delta+2 \sigma) \tilde{\Gamma}}{\left(\delta+\sigma-\mu_{1}\right)\left(\delta+\sigma-\mu_{2}\right)\left(\omega_{3}^{2}-\omega_{3}^{1}\right)} \\
& \times\left[\mu_{1} \mu_{2}\left(\mu_{1} \omega_{3}^{2}-\mu_{2} \omega_{3}^{1}\right)-(\delta+\sigma)\left(\mu_{1}^{2} \omega_{3}^{2}-\mu_{2}^{2} \omega_{3}^{1}\right)\right] \frac{\mathrm{d} \tilde{s}}{\mathrm{~d} g^{j}}<0,
\end{aligned}
$$

where $\mu_{1}^{2} \omega_{3}^{2}-\mu_{2}^{2} \omega_{3}^{1}>0$ since $\omega_{3}^{2}>\omega_{3}^{1}$ and $\mu_{1}^{2}>\mu_{2}^{2}$. After its upward jump, the time preference decreases. Performing a similar procedure than previously, it is straightforward to show that the possibility of a non-monotonic adjustment rests on the condition of an initial positive change of the time preference rate. Since this condition does not hold (see (172)), we can infer that the time preference rate, $\rho(t)$, decreases monotonically along the stable trajectory. 


\section{E Welfare Analysis}

In this section, we investigate the welfare effects of an unanticipated permanent rise in government spending, $g^{j}$, falling on the domestic good $(i=d)$ or the foreign good $(i=f)$. We denote by $\phi$ the instantaneous welfare:

$$
\phi(t)=u(c(t), s(t))
$$

and by $U$ its discounted value over an infinite horizon:

$$
U=\int_{0}^{\infty} \phi(t) \exp (-\delta t) \mathrm{d} t
$$

From (10a), the steady-state value of real consumption is given by:

$$
\tilde{c}=\left[\left(\frac{\delta+\sigma}{\delta+\sigma(1-\gamma)}\right) \frac{p_{c} \bar{\lambda}}{\tilde{p}}\right]^{-\frac{1}{\gamma+\epsilon(1-\gamma)}} .
$$

Differentiating the felicity function (??) w. r. t. $\quad c$ and $s$ and evaluate at the steady state using (175), this yields to:

$$
\begin{aligned}
& \tilde{u}_{c}=\tilde{c}^{-[\gamma+\epsilon(1-\gamma)]}=\left(\frac{\delta+\sigma}{\delta+\sigma(1-\gamma)}\right) \frac{p_{c} \bar{\lambda}}{\tilde{p}}>0, \\
& \tilde{u}_{s}=-\gamma \tilde{c}^{-[\gamma+\epsilon(1-\gamma)]}=-\gamma\left(\frac{\delta+\sigma}{\delta+\sigma(1-\gamma)}\right) \frac{p_{c} \bar{\lambda}}{\tilde{p}}=-\gamma \tilde{u}_{c}<0,
\end{aligned}
$$

where a tilde over partial derivatives indicate that they are evaluated at the steady-state. Furthermore, we have computed several useful expressions:

$$
\begin{aligned}
& \Delta_{1} \equiv \tilde{u}_{c}\left(\frac{\sigma+\mu_{1}}{\sigma}\right)+\tilde{u}_{s}=\left(\frac{\delta+\sigma}{\delta+\sigma(1-\gamma)}\right) \frac{p_{c} \bar{\lambda}}{\tilde{p}}\left[\frac{\sigma(1-\gamma)+\mu_{1}}{\sigma}\right]<0 \\
& \Delta_{2} \equiv \tilde{u}_{c}\left(\frac{\sigma+\mu_{2}}{\sigma}\right)+\tilde{u}_{s}=\left(\frac{\delta+\sigma}{\delta+\sigma(1-\gamma)}\right) \frac{p_{c} \bar{\lambda}}{\tilde{p}}\left[\frac{\sigma(1-\gamma)+\mu_{2}}{\sigma}\right]>0
\end{aligned}
$$

where we imposed the following condition which holds from plausible values of parameters (as indicated by numerical analysis):

$$
\sigma(1-\gamma)+\mu_{1}<0, \quad \sigma(1-\gamma)+\mu_{2}>0
$$

\section{E.1 Instantaneous Welfare Effects}

We first linearize the instantaneous utility function (??) in the neighborhood of the steadystate:

$$
\phi(t)=\tilde{\phi}+u_{c}(\tilde{c}, \tilde{s})(c(t)-\tilde{c})+u_{s}(\tilde{c}, \tilde{s})(s(t)-\tilde{s}),
$$


with $\tilde{\phi}$ given by

$$
\tilde{\phi}=u(\tilde{c}, \tilde{c})
$$

where we used the fact $\tilde{c}=\tilde{s}$.

By substituting solutions for $s(t)$ and $c(t)$, we obtain the two-dimensional stable solution for instantaneous welfare:

$$
\phi(t)=\tilde{\phi}+\left[\tilde{u}_{c}\left(\frac{\sigma+\mu_{1}}{\sigma}\right)+\tilde{u}_{s}\right] A_{1} e^{\mu_{1} t}+\left[\tilde{u}_{s}\left(\frac{\sigma+\mu_{2}}{\sigma}\right)+\tilde{u}_{s}\right] A_{2} e^{\mu_{2} t}
$$

where a tilde indicates that the variable is evaluated at the steady-state, that is $\tilde{u}_{c}=u_{c}(\tilde{c}, \tilde{c})$ and $\tilde{u}_{s}=u_{s}(\tilde{c}, \tilde{c})$.

We investigate the initial reaction of instantaneous welfare. We evaluate (181) at time $t=0$ and differentiate this w. r. t. $g^{j}$ :

$$
\begin{aligned}
\frac{\mathrm{d} \phi(0)}{\mathrm{d} g^{j}} & =\left(\tilde{u}_{c}+\tilde{u}_{s}\right) \frac{\mathrm{d} \tilde{s}}{\mathrm{~d} g^{j}}+\Delta_{1} \frac{A_{1}}{\mathrm{~d} g^{j}}+\Delta_{2} \frac{A_{2}}{\mathrm{~d} g^{j}}, \\
& =-\tilde{u}_{c}\left[\frac{\mu_{1} \omega_{3}^{2}-\mu_{2} \omega_{3}^{1}}{\sigma\left(\omega_{3}^{2}-\omega_{3}^{1}\right)}\right] \frac{\mathrm{d} \tilde{s}}{\mathrm{~d} g^{j}}=\tilde{u}_{c} \frac{\mathrm{d} c(0)}{\mathrm{d} g^{j}}<0, \quad i=d, f,
\end{aligned}
$$

where $\mu_{1} \omega_{3}^{2}-\mu_{2} \omega_{3}^{1}<0$ (see (146b)) and $\omega_{3}^{2}-\omega_{3}^{1}>0$ (see (46)). The last equality has been obtained by inspecting expression (153) that gives the initial reaction of real consumption.

Differentiate (180) w. r. t. $g^{j}$, one obtains the long-run change of instantaneous welfare:

$$
\frac{\mathrm{d} \tilde{\phi}}{\mathrm{d} g^{j}}=\left(\tilde{u}_{c}+\tilde{u}_{s}\right) \frac{\mathrm{d} \tilde{c}}{\mathrm{~d} g^{j}}<0, \quad i=d, f .
$$

As longer as $0 \leq \gamma<1$, an unexpected permanent fiscal expansion (regardless of the good on which the fiscal expansion falls) induces a fall in the steady-state value of $\phi$ triggered by the long-run fall in real consumption.

By making use of (182) and (183), we make the comparison between the size of initial change of $\phi$ and the size of its long-run change:

$$
\frac{\mathrm{d} \phi(0)}{\mathrm{d} g^{j}}-\frac{\mathrm{d} \tilde{\phi}}{\mathrm{d} g^{j}}=-\tilde{u}_{c}\left\{\frac{\omega_{3}^{2}\left[\sigma(1-\gamma)+\mu_{1}\right]-\omega_{3}^{1}\left[\sigma(1-\gamma)+\mu_{2}\right]}{\sigma\left(\omega_{3}^{2}-\omega_{3}^{1}\right)}\right\} \frac{\mathrm{d} \tilde{s}}{\mathrm{~d} g^{j}}<0, \quad i=d, f .
$$

According to (184), following a fiscal expansion, instantaneous welfare falls initially by a larger amount than in the long-run. This is due to the fact that the habit stock is initially predetermined and does not change at time $t=0$. 
Along the transitional path, $\phi$ rises towards its new long-run level:

$$
\dot{\phi}(t)=\mu_{1} \Delta_{1} \frac{A_{1}}{\mathrm{~d} g^{j}} \mathrm{~d} g^{j} e^{\mu_{1} t}+\mu_{2} \Delta_{2} \frac{A_{2}}{\mathrm{~d} g^{j}} \mathrm{~d} g^{j} e^{\mu_{2} t}>0, \quad i=d, f,
$$

where we took the time derivative of the the stable solution for $\phi(t)$ (given by (181)). The sign follows from the fact that $\Delta_{1}<0$ and $\Delta_{2}>0$. From (185), along the stable adjustment, instantaneous welfare rises towards its new lower long-run level.

According to (184), whatever the size of the weight $\gamma$ attached to the reference stock, $s$, the short-run response of $\phi$ overshoots its new steady-state value, i. e. $\phi(0)<\tilde{\phi}$. Since the habit stock is initially predetermined, $s(0)=s_{0}$, the initial change of instantaneous welfare is only driven by the variation of real consumption at time $t=0$ which induces welfare losses. Over time, the positive impact of the gradual fall in the habit stock upon $\phi$ more than outweighs the negative impact of the progressive decrease in $c$. This effect in reflected by the first term on the right-hand side of (185). As we have shown previously (in section D.4), there exists a time at which the real consumption together with the consumption experience start increasing. Reflected by the second term on the right-hand side of (185), this adjustment has a positive impact on instantaneous welfare.

\section{E.2 Overall Welfare Effects}

Until now, we have analyzed the instantaneous welfare implications of an unanticipated permanent fiscal expansion, say at different points of times. To address welfare effects in a convenient way within an intertemporal-maximizing framework, we have to evaluate the discounted value of (173) over the agent's infinite planning horizon. We will see that the sluggish adjustment in welfare that arises from consumption inertia enters in sharp contrast with the immediate jump of welfare prevailing with time separable preferences.

In order to have a correct and comprehensive measure of welfare, we calculate first the discounted value of instantaneous welfare over the entire planning horizon

$$
U=\frac{\tilde{u}}{\delta}+\frac{\left[\tilde{u}_{c}\left(\frac{\sigma+\mu_{1}}{\sigma}\right)+\tilde{u}_{s}\right]}{\delta-\mu_{1}} A_{1}+\frac{\left[\tilde{u}_{c}\left(\frac{\sigma+\mu_{2}}{\sigma}\right)+\tilde{u}_{s}\right]}{\delta-\mu_{2}} A_{2} .
$$


The first term on the right hand-side of (186) represents the capitalized value of instantaneous welfare evaluated at the steady-state. The second and the third term on the right hand-side of (186) vanish whenever preferences are time separable.

In order to derive the overall (des)accumulated welfare following an unanticipated permanent increase of government expenditure, we differentiate (178) w.r.t $g^{j}$ :

$$
\begin{aligned}
\frac{\mathrm{d} U}{\mathrm{~d} g^{j}} & =\frac{\tilde{u}_{c}+\tilde{u}_{s}}{r^{\star}} \frac{\mathrm{d} \tilde{s}}{\mathrm{~d} g^{j}}-\frac{\omega_{3}^{2}}{\omega_{3}^{2}-\omega_{3}^{1}} \frac{\left[\tilde{u}_{c}\left(\frac{\sigma+\mu_{1}}{\sigma}\right)+\tilde{u}_{s}\right]}{\left(r^{\star}-\mu_{1}\right)} \frac{\mathrm{d} \tilde{s}}{\mathrm{~d} g^{j}} \\
& +\frac{\omega_{3}^{1}}{\omega_{3}^{2}-\omega_{3}^{1}} \frac{\left[\tilde{u}_{c}\left(\frac{\sigma+\mu_{2}}{\sigma}\right)+\tilde{u}_{s}\right]}{\left(r^{\star}-\mu_{2}\right)} \frac{\mathrm{d} \tilde{s}}{\mathrm{~d} g^{j}} \\
& =\left[\frac{\mu_{2} \mu_{4} \omega_{3}^{1}-\mu_{1} \mu_{3} \omega_{3}^{2}}{r^{\star} \mu_{3} \mu_{4}\left(\omega_{3}^{2}-\omega_{3}^{1}\right)}\right]\left[\left(1+\frac{\delta}{\sigma}\right) \tilde{u}_{c}+\tilde{u}_{s}\right] \frac{\mathrm{d} \tilde{s}}{\mathrm{~d} g^{j}}<0,
\end{aligned}
$$

where we have evaluated the following expression to determine the sign of (187)

$$
\begin{aligned}
& \mu_{2} \mu_{4} \omega_{3}^{1}-\mu_{1} \mu_{3} \omega_{3}^{2} \\
= & -r^{\star}\left(\mu_{1} \omega_{3}^{2}-\mu_{2} \omega_{3}^{1}\right)+\mu_{1} \mu_{2}\left(\omega_{3}^{2}-\omega_{3}^{1}\right) \\
= & \frac{I_{c} \mu_{1} \mu_{2}\left(\mu_{1}-\mu_{2}\right)\left\{\left(\sigma+\mu_{1}\right)\left[\mu_{3} \mu_{4}+I_{p} \tilde{p} F_{k k}\right]-\mu_{3}\left[\mu_{1} \mu_{4}+I_{p} \tilde{p} F_{k k}\right]\right\}}{\sigma\left[\mu_{1} \mu_{4}+I_{p} \tilde{p} F_{k k}\right]\left[\mu_{2} \mu_{3}+I_{p} \tilde{p} F_{k k}\right]}, \\
= & \frac{(+)}{(+)}>0,
\end{aligned}
$$

where we have substituted expressions (46) and (146b) to determine (188). From (187), a permanent rise in government spending, $g^{j}$, leads to a reduction of total welfare.

The long-term change in total welfare is equal to

$$
\frac{\mathrm{d} \tilde{U}}{\mathrm{~d} g^{j}}=\frac{\tilde{u}_{c}+\tilde{u}_{s}}{\delta} \frac{\mathrm{d} \tilde{s}}{\mathrm{~d} g^{j}}<0
$$

where $\delta=r^{\star}$ and $\tilde{u}_{c}+\tilde{u}_{s}>0$ according to assumption [H3] described in section 2 .

We turn now to the computation of the two-dimensional stable solution for total welfare. At some date $t$, total welfare is given by

$$
U(t)=\int_{t}^{\infty} \phi(t) e^{-\delta(\nu-t)} \mathrm{d} \nu
$$

Substituting the solution for $\phi(t)$ and integrating, we get

$$
U(t)-\tilde{U}=\frac{\left[\tilde{u}_{c}\left(\frac{\sigma+\mu_{1}}{\sigma}\right)+\tilde{u}_{s}\right]}{\delta-\mu_{1}} A_{1} e^{\mu_{1} t}+\frac{\left[\tilde{u}_{c}\left(\frac{\sigma+\mu_{2}}{\sigma}\right)+\tilde{u}_{s}\right]}{\delta-\mu_{2}} A_{2} e^{\mu_{2} t}
$$


where we used the fact that $\tilde{U}=\tilde{u} / \delta$. Differentiate (191) w. r. t. to time $t$, total welfare evolves as follows along the stable trajectory after an unanticipated permanent fiscal expansion:

$$
\dot{U}(t)=\underbrace{\mu_{1} \frac{\left[\tilde{u}_{c}\left(\frac{\sigma+\mu_{1}}{\sigma}\right)+\tilde{u}_{s}\right]}{\delta-\mu_{1}} \frac{A_{1}}{\mathrm{~d} g^{j}}}_{(+)} \mathrm{d} g^{j} e^{\mu_{1} t}+\underbrace{\frac{\left[\tilde{u}_{c}\left(\frac{\sigma+\mu_{2}}{\sigma}\right)+\tilde{u}_{s}\right]}{\delta-\mu_{2}} \frac{A_{2}}{\mathrm{~d} g^{j}}}_{(+)} \mathrm{d} g^{j} e^{\mu_{2} t}>0,
$$

where $A_{1} / \mathrm{d} g^{j}>0$ and $A_{2} / \mathrm{d} g^{j}<0$ are given by $(75),\left[\tilde{u}_{c}\left(\frac{\sigma+\mu_{1}}{\sigma}\right)+\tilde{u}_{s}\right]<0$ and $\left[\tilde{u}_{c}\left(\frac{\sigma+\mu_{2}}{\sigma}\right)+\tilde{u}_{s}\right]>0$ are given by (177). From (187), (189) and (192), overall welfare falls abruptly at time $t=0$, then rises towards its new lower long-run level, $\tilde{U}$.

\section{F Savings}

\section{Formal Solution for the Stock of Financial Wealth}

Financial wealth measured in terms of the domestic good, $p(t) a(t)$, is equal to the sum of the stock of foreign assets measured in terms of the foreign good, $p(t) b(t)$, and the capital stock. The law of motion for financial wealth $(S(t)=\dot{a}(t))$ is given by:

$$
p(t) \dot{a}(t)=r^{\star} p(t) a(t)+w-p_{c}(p(t)) c-Z,
$$

with $Z=g^{D}+p g^{f}$. Linearize (193) in the neighborhood of the steady-state:

$$
\begin{aligned}
\tilde{p} \dot{a}(t) & =r^{\star} \tilde{p}(a(t)-\tilde{a})-p_{c}(c(t)-\tilde{c})-\frac{\left(w-\tilde{c}^{D}-g^{D}\right)}{\tilde{p}}(p(t)-\tilde{p}), \\
& =r^{\star} \tilde{p}(a(t)-\tilde{a})-p_{c}(c(t)-\tilde{c})+\left(r^{\star} \tilde{a}-\tilde{c}^{F}+g^{F}\right)(p(t)-\tilde{p}),
\end{aligned}
$$

where $w \equiv F(k)-F_{k}(k)$ and $\left(w-\tilde{c}^{D}-g^{D}\right)=\tilde{p}\left(\tilde{c}^{F}+g^{F}-r^{\star} \tilde{a}\right)$.

Inserting the stable solutions for $(p(t)-\tilde{p})$ and $(c(t)-\tilde{c})$ given respectively by $(27 \mathrm{~d})$ and (27b), the solution for the current account writes as follows :

$$
\tilde{p} \dot{a}(t)=r^{\star} \tilde{p}(a(t)-\tilde{a})+\tilde{\Upsilon} \sum_{i=1}^{2} A_{i} \omega_{4}^{i} e^{\mu_{i} t}-p_{c} \sum_{i=1}^{2} A_{i} \omega_{2}^{i} e^{\mu_{i} t}
$$

where we set $\tilde{\Upsilon}=\left(r^{\star} \tilde{a}-\tilde{c}^{F}+g^{F}\right)$.

Solving the differential equation leads to:

$$
a(t)-\tilde{a}=\left[\left(a_{0}-\tilde{a}\right)-\frac{S_{1} A_{1}}{\mu_{1}-r^{\star}}-\frac{S_{2} A_{2}}{\mu_{2}-r^{\star}}\right] e^{r^{\star} t}+\frac{S_{1} A_{1}}{\mu_{1}-r^{\star}} e^{\mu_{1} t}+\frac{S_{2} A_{2}}{\mu_{2}-r^{\star}} e^{\mu_{2} t},
$$


where

$$
S_{1}=\frac{1}{\tilde{p}}\left\{\tilde{\Upsilon} \omega_{4}^{1}-p_{c} \omega_{2}^{1}-F_{k k} \tilde{k} \omega_{3}^{1}\right\}, \quad S_{2}=\frac{1}{\tilde{p}}\left\{\tilde{\Upsilon} \omega_{4}^{2}-p_{c} \omega_{2}^{2}-F_{k k} \tilde{k} \omega_{3}^{2}\right\}
$$

Dynamics of savings is the result of conflictory forces such that the signs of $S_{1}$ and $S_{2}$ remain undetermined. Over a first phase, the three effects play in the same direction and induce a decumulation of financial wealth. First, as longer as $\tilde{\Upsilon}$ is positive, the real exchange rate depreciation lowers financial wealth by reducing the real wage. Second, as longer as consumption remains higher than its steady-state level, this consumption behavior impinges negatively on savings. Third, a transitorily lower capital stock raised the marginal production of physical capital and reduces the wage. At some date, real consumption equalizes its steady-state value from above. This overshooting behavior impinges positively on savings and may eventually more than outweighs the two other effects such that households accumulate financial wealth.

Invoking the transversality condition for intertemporal solvency, we obtain the linearized version of the nation's intertemporal budget constraint:

$$
a_{0}-\tilde{a}=\frac{S_{1} A_{1}}{\mu_{1}-r^{\star}}+\frac{S_{2} A_{2}}{\mu_{2}-r^{\star}}
$$

For the national intertemporal solvency to hold, the terms in brackets of equation (196) must be null, so the stable solution for net foreign assets finally reduces to

$$
a(t)-\tilde{a}=\frac{S_{1} A_{1}}{\mu_{1}-r^{\star}} e^{\mu_{1} t}+\frac{S_{2} A_{2}}{\mu_{2}-r^{\star}} e^{\mu_{2} t}
$$

\section{G Functional Forms: Some Analytical Expressions}

\section{G.1 Functional Forms and the Steady-State}

\section{Consumption-Side}

The utility function is of the CRRA form:

$$
u(c, s)=\frac{1}{1-\epsilon}\left(\frac{c}{s^{\gamma}}\right)^{1-\epsilon},
$$

where the parameter $\epsilon$ corresponds to the coefficient of relative risk aversion and the parameter $\gamma$ indexes the importance of habit formation in the instantaneous utility function. 
First and second partial derivatives of the instantaneous utility function are as follows:

$$
\begin{aligned}
& u_{c}=c^{-\epsilon} s^{-\gamma(1-\epsilon)}>0 \\
& u_{s}=-\gamma c^{1-\epsilon} s^{-[\gamma(1-\epsilon)+1]}<0 \\
& u_{c c}=-\epsilon c^{-(1+\epsilon)} s^{-\gamma(1-\epsilon)}<0 \\
& u_{s s}=\gamma[\gamma(1-\epsilon)+1] c^{1-\epsilon} s^{-[\gamma(1-\epsilon)+2]}<0 \text { if and only if } \epsilon>\frac{1+\gamma}{\gamma}, \\
& u_{c s}=-\gamma(1-\epsilon) c^{-\epsilon} s^{-[\gamma(1-\epsilon)+1]}>0 \text { if and only if } \epsilon>1 .
\end{aligned}
$$

We give some useful expressions using the functional form: (200):

$$
\begin{aligned}
& \tilde{u}_{c}+\frac{\sigma}{\delta+\sigma} \tilde{u}_{s}=\tilde{c}^{-[\epsilon+\gamma(1-\epsilon)]} \frac{\delta+\sigma(1-\gamma)}{\delta+\sigma}>0, \\
& \frac{\sigma(\delta+\sigma)}{\tilde{u}_{11}}\left[\tilde{u}_{11}+\frac{\delta+2 \sigma}{\delta+\sigma} \tilde{\Gamma}\right]=\frac{\sigma}{\epsilon}[\gamma+\epsilon(1-\gamma)][\delta+\sigma(1-\gamma)]>0, \\
& -\frac{\left[\tilde{u}_{c}+\frac{\sigma}{\delta+\sigma} \tilde{u_{s}}\right]}{\left[\tilde{u}_{11}+\frac{\delta+2 \sigma}{\delta+\sigma} \tilde{\Gamma}\right] \tilde{c}}=1 /[\gamma+\epsilon(1-\gamma)], \\
& -\frac{p_{c} \bar{\lambda}}{\tilde{p} \tilde{u}_{11} \tilde{c}}=-\frac{\tilde{u}_{c}+\frac{\sigma}{\delta+\sigma} \tilde{u}_{s}}{\tilde{u}_{11} \tilde{c}}=\frac{[\delta+\sigma(1-\gamma)]}{\epsilon(\delta+\sigma)}>0, \\
& -\frac{\sigma(\delta+2 \sigma)}{\tilde{u}_{11}} \tilde{\Gamma}=\frac{\sigma \gamma}{\epsilon}\{\sigma \epsilon-(1-\epsilon)[\delta+\sigma(1-\gamma)]\}>0 .
\end{aligned}
$$

where a tilde indicates that the instantaneous utility is evaluated at the steady state, $\tilde{c}=\tilde{s}$, and $\Gamma$ is defined by (??).

We assume that the representative household maximizes a CES function given by:

$$
c(., .)=\left[\varphi^{\frac{1}{\phi}}\left(c^{D}\right)^{\frac{\phi-1}{\phi}}+(1-\varphi)^{\frac{1}{\phi}}\left(c^{F}\right)^{\frac{\phi-1}{\phi}}\right]^{\frac{\phi}{\phi-1}}
$$

with $\phi>0$ the intratemporal elasticity of substitution between consumption of domestic and foreign goods, given total expenditure measured in terms of traded goods:

$$
E \equiv c^{D}+p c^{f}
$$

At the first stage, the household minimizes the cost, $E(t)=c^{D}(t)+p(t) c^{F}(t)$, for a given level of subutility, $c(t)$, where $p(t)$ is the relative price of the foreign good or the real exchange rate. For any chosen $c(t)$, the optimal basket $\left(c^{D}(t), c^{F}(t)\right)$ is a solution to:

$$
p_{c}(p(t)) c(t)=\min _{\left\{c^{D}(t), c^{F}(t)\right\}}\left\{c^{D}(t)+p(t) c^{F}(t)(t): c\left(c^{D}(t), c^{F}(t)\right) \geq c(t)\right\}
$$


The subutility function $c($.$) is linear homogeneous implies that the total expense in consump-$ tion goods can be expressed as $E(t)=p_{c}(p(t)) c(t)$, with $p_{c}(p(t))$ is the unit cost function dual (or consumption-based price index) to $c$. The unit cost dual function, $p_{c}($.$) , is defined as$ the minimum total expense in consumption goods, $E$, such that $c=c\left(c^{D}(t), c^{F}(t)\right)=1$, for a given level of the real exchange rate, $p$. Its expression is given by

$$
p_{c}=\left[\varphi+(1-\varphi) p^{1-\phi}\right]^{\frac{1}{1-\phi}}
$$

The minimized unit cost function depends on the real exchange rate and is expressed in terms of the domestic good. It has the following properties:

$$
\begin{aligned}
p_{c}^{\prime} & =(1-\varphi) p^{-\phi}\left[\varphi+(1-\varphi) p^{1-\phi}\right]^{\frac{\phi}{1-\phi}}>0 \\
p_{c}^{\prime \prime} & =\phi(1-\varphi) p^{-(1+\phi)}\left[\varphi+(1-\varphi) p^{1-\phi}\right]^{\frac{\phi}{1-\phi}}\left[\frac{(1-\varphi) e^{1-\phi}}{\varphi+(1-\varphi) p^{1-\phi}}-1\right]<0 \\
& =\phi p_{c}^{\prime} p^{-1}\left[\frac{(1-\varphi) e^{1-\phi}}{\varphi+(1-\varphi) p^{1-\phi}}-1\right]
\end{aligned}
$$

Intra-temporal allocations between non tradable goods and tradable goods follow from Sheppard's Lemma (or the envelope theorem) applied to (205):

$$
\begin{aligned}
c^{F} & =p_{c}^{\prime} c, \text { and } \frac{p c^{f}}{p_{c} c}=\alpha_{c}, \\
c^{D} & =\left[p_{c}-p p_{c}^{\prime}\right] c, \text { and } \frac{c^{D}}{p_{c} c}=\left(1-\alpha_{c}\right),
\end{aligned}
$$

with the shares of the foreign and the domestic goods in consumption expenditure are given respectively by

$$
\begin{aligned}
\alpha_{c} & =\frac{(1-\varphi) p^{1-\phi}}{\varphi+(1-\varphi) p^{1-\phi}}, \\
1-\alpha_{c} & =\frac{\varphi}{\varphi+(1-\varphi) p^{1-\phi}} .
\end{aligned}
$$

By combining equations (207b) and (210), we can rewrite the term $-p_{c}^{\prime \prime} p / p_{c}^{\prime}$ as follows:

$$
-\frac{p_{c}^{\prime \prime} p}{p_{c}^{\prime}}>0
$$

where $\phi\left(1-\alpha_{c}\right)$ represents the elasticity of consumption in the foreign good with respect to the real exchange rate. 


\section{Production-Side}

We assume that the production function takes the Cobb-Douglas form:

$$
Y=f(k)=k^{\alpha_{K}},
$$

where $\alpha_{K}$ corresponds to the capital share.

\section{Export Function}

As in Turnovsky and Sen [?], the country may be able to influence the real exchange rate. This influence is captured by an export function, $X(p)$ which takes a power form:

$$
X=X(p)=\gamma_{X} p^{\nu X}
$$

where $\gamma_{X}>0$ is a constant and $\nu_{X}$ represents the price elasticity of the home country's export. Note that in the limiting case, of a perfectly elastic export demand function, i. e. $\nu_{X}=+\infty$, the real exchange rate is equal to unity.

By making use of the functional form for the export function (212), we can rewrite the partial derivative of the investment function with respect to the real exchange rate:

$$
\begin{aligned}
I_{p} & =-\left(X_{p}-p p_{c}^{\prime \prime} c\right), \\
& =-\left[\nu_{X}(X / p)+\phi\left(1-\alpha_{c}\right) c^{F}\right]<0,
\end{aligned}
$$

where we made use of (210).

Finally, we evaluate at the steady-state the net export reaction function with respect to the real exchange rate (given by (38)) by substituting the functional form for the export function (eq (212)):

$$
\tilde{\Omega} \equiv-\frac{1}{\tilde{p}}\left[I_{p}+\frac{\tilde{X}}{\tilde{p}}\right]=\frac{\tilde{X}}{\tilde{p}^{2}}\left[\nu_{X}+\phi\left(1-\alpha_{c}\right) \frac{\tilde{p} \tilde{c}^{F}}{\tilde{X}}-1\right]>0 .
$$

\section{Steady-State}


The steady-state (10) can be rewritten as follows:

$$
\begin{gathered}
\tilde{c}=\left[\left(\frac{\delta+\sigma}{\delta+\sigma(1-\gamma)}\right) \frac{p_{c} \bar{\lambda}}{\tilde{p}}\right]^{-\frac{1}{\gamma+\epsilon(1-\gamma)}}, \\
\tilde{c}=\tilde{s} \\
\tilde{k}=\left(\frac{\alpha_{K}}{r^{\star}}\right)^{\frac{1}{1-\alpha_{K}}}, \\
\tilde{k}^{\alpha_{K}}=\left(1-\alpha_{c}\right) p_{c}(\tilde{p}) \tilde{c}+X_{0}(\tilde{p})^{\nu_{X}}+g^{D}, \\
\tilde{b}=-\left[\frac{\tilde{k}^{\alpha_{K}}-p_{c}(\tilde{p}) \tilde{c}-g^{D}-\tilde{p} g^{F}}{\tilde{p} r^{\star}}\right], \\
\tilde{b}-\Phi_{1} \tilde{k}-\Phi_{2} \tilde{s}=b_{0}-\Phi_{1} k_{0}-\Phi_{2} s_{0} .
\end{gathered}
$$

By using the functional form (200) for preferences, the short-run static condition (??) determines the steady-state value of real consumption as we can see from (215a) which indicates that $\tilde{c}$ depends on the equilibrium value of the marginal utility of wealth and the real exchange rate. From (215b), the reference stock must coincide with the real consumption in the longrun. The equality between the marginal product of physical capital and the world interest rate allows to determine the long-term level of $k$. To obtain expression (215c) of the steady-state value of the capital stock, we made use of the functional form for production given by (211).

Equation (224) corresponds to the steady-state value of the real exchange rate which is determined by the means of the market-clearing condition (10b) together with the production function (211) and export function (212). Consumption of the domestic good, $\tilde{c}^{D}=\left(1-\alpha_{c}\right) p_{c}(\tilde{p}) \tilde{c}$ is obtained by combining (209b), (206) and (215a). Expression (215d) shows that the long-term value of the real exchange rate depends on the steady-state values of the physical capital stock and the real consumption given respectively by (215c) and (215a).

The current account equilibrium in the long-run allows for determining the stock of traded bonds given by (215e) as a function of: the steady-state endogenous values like the real consumption, $\tilde{c}$, the real exchange rate, $\tilde{p}$ and the capital stock $\tilde{k}$ on the one hand, and on the other hand the exogenous variables like government spending on the domestic good, $g^{D}$ and the foreign good, $g^{F}$, and the world interest rate, $r^{\star}$.

Independently from the linearized version of the intertemporal solvency condition (215f), 
the system of equations (215a) and (215e) can be solved for $\bar{\lambda}$ and the government policy parameters, $g^{D}$ and $g^{F}$. Substituting the steady-state values of the stock of habits, i. e. $\tilde{s}=$ $\tilde{c}=m\left(\bar{\lambda}, g^{D}, g^{F}\right)$, and the long-term value of the stock of international assets, i. e. $\tilde{b}=$ $b\left(\bar{\lambda}, g^{D}, g^{F}\right)$ into the solvency condition (215f), we are able to determine the equilibrium value of the marginal utility of wealth such that (215f) holds.

Furthermore, the steady-state values of consumption in the domestic good and the foreign good can be written as follows:

$$
\begin{aligned}
\tilde{c}^{D} & =\left(1-\alpha_{c}\right) p_{c} \tilde{c}=\varphi\left[\varphi+(1-\varphi) \tilde{p}^{1-\phi}\right]^{\frac{\phi}{1-\phi}} \tilde{c} \\
\tilde{c}^{F} & =\frac{\alpha_{c} p_{c} \tilde{c}}{\tilde{p}}=(1-\varphi) \tilde{p}^{-\phi}\left[\varphi+(1-\varphi) \tilde{p}^{1-\phi}\right]^{\frac{\phi}{1-\phi}} \tilde{c}
\end{aligned}
$$

\section{G.2 Eigenvectors and Real Eigenvalues}

\section{The Linearized Matrix}

Making use of functional forms described in section G.1, the linearized matrix (15) can be rewritten as follows:

$$
J \equiv\left(\begin{array}{cccc}
-\sigma & \sigma & 0 & 0 \\
a_{21} & \delta+\sigma & a_{23} & a_{24} \\
0 & -\left(1-\alpha_{c}\right) p_{c} & r^{\star} & a_{34} \\
0 & 0 & \tilde{p} F_{k k} & 0
\end{array}\right)
$$

with

$$
\begin{aligned}
& a_{21}=-\frac{\gamma}{\epsilon}\{\sigma \epsilon-(1-\epsilon)[\delta+\sigma(1-\gamma)]\},<0, \\
& a_{23}=\frac{[\delta+\sigma(1-\gamma)]}{\epsilon(\delta+\sigma)} \frac{\tilde{c}^{D} F_{k k}}{p_{c}}>0, \\
& a_{24}=-\frac{[\delta+\sigma(1-\gamma)]}{\epsilon} \frac{\left(1-\alpha_{c}\right) \tilde{c}}{\tilde{p}}<0 . \\
& a_{34}=-\frac{1}{\tilde{p}}\left[\nu_{X} \tilde{X}+\phi\left(1-\alpha_{c}\right) \tilde{p} \tilde{c}^{F}\right]<0 .
\end{aligned}
$$

\section{Determinant and Condition for Real Roots}

The determinant $J$ of the matrix of the linearized system denoted by $b_{4}$ (given by (19d)) can be rewritten as by making use of the functional forms for the production function, the 
export function, and real consumptio aggregator function:

$$
\text { Det } \mathrm{J}=b_{4}=\frac{\sigma}{\epsilon}[\delta+\sigma(1-\gamma)] F_{k k}\left\{[\gamma+\epsilon(1-\gamma)] I_{p} \tilde{p}-\left(1-\alpha_{c}\right) \tilde{c}^{D}\right\}>0
$$

where we have substituted $(202 \mathrm{~b}),(202 \mathrm{~d})$, and used the fact that $I_{c}=-\left(1-\alpha_{c}\right) p_{c}$ and $\tilde{c}^{D}=\left(1-\alpha_{c}\right) p_{c} \tilde{c}$. Performing a similar procedure, we can evaluate the term $b_{3} / r^{\star}$ (given by $(19 \mathrm{c}))$ :

$$
\frac{b_{3}}{r^{\star}}=\frac{\sigma}{\epsilon}[\gamma+\epsilon(1-\gamma)][\delta+\sigma(1-\gamma)]+F_{k k}\left[\tilde{p} I_{p}-\left(1-\alpha_{c}\right) \tilde{c}^{D} \frac{[\delta+\sigma(1-\gamma)]}{\epsilon(\delta+\sigma)}\right]>0 .(220)
$$

and the condition (26) for real roots:

$$
\begin{aligned}
\left(\frac{b_{3}}{r^{\star}}\right)^{2}-4 b_{4} & =\left\{\frac{\sigma}{\epsilon}[\gamma+\epsilon(1-\gamma)][\delta+\sigma(1-\gamma)]-F_{k k}\left[\tilde{p} I_{p}-\left(1-\alpha_{c}\right) \tilde{c}^{D} \frac{[\delta+\sigma(1-\gamma)]}{\epsilon(\delta+\sigma)}\right]\right\}^{2} \\
& +4 \frac{\sigma \gamma}{\epsilon}\{\sigma \epsilon-(1-\epsilon)[\delta+\sigma(1-\gamma)]\} \frac{[\delta+\sigma(1-\gamma)]}{\epsilon(\delta+\sigma)} F_{k k}\left(1-\alpha_{c}\right) \tilde{c}^{D} \gtrless 0, \quad(221)
\end{aligned}
$$

with $I_{p}$ given by equation (213) and $F_{k k}$ evaluated at the steady-state can be written as follows:

$$
F_{k k}=-\frac{r^{\star}\left(1-\alpha_{K}\right)}{\tilde{k}}
$$

\section{Eigenvalues}

We write out the two stable and the two unstable eigenvalues:

$$
\begin{aligned}
& \mu_{1} \equiv \frac{1}{2}\left\{r^{\star}-\sqrt{\left(r^{\star}\right)^{2}+2\left(\frac{b_{3}}{r^{\star}}+\sqrt{\left.\left(\frac{b_{3}}{r^{\star}}\right)^{2}-4 b_{4}\right)}\right\}<0,}\right. \\
& \mu_{2} \equiv \frac{1}{2}\left\{r^{\star}-\sqrt{\left(r^{\star}\right)^{2}+2\left(\frac{b_{3}}{r^{\star}}-\sqrt{\left.\left(\frac{b_{3}}{r^{\star}}\right)^{2}-4 b_{4}\right)}\right\}<0,}\right. \\
& \mu_{3} \equiv \frac{1}{2}\left\{r^{\star}+\sqrt{\left(r^{\star}\right)^{2}+2\left(\frac{b_{3}}{r^{\star}}-\sqrt{\left(\frac{b_{3}}{r^{\star}}\right)^{2}-4 b_{4}}\right)}\right\}>0, \\
& \mu_{4} \equiv \frac{1}{2}\left\{r^{\star}+\sqrt{\left(r^{\star}\right)^{2}+2\left(\frac{b_{3}}{r^{\star}}+\sqrt{\left(\frac{b_{3}}{r^{\star}}\right)^{2}-4 b_{4}}\right)}\right\}>0,
\end{aligned}
$$

where $b_{3} / r^{\star},\left(b_{3} / r^{\star}\right)^{2}-4 b_{4}$ are given by (220) and (221) respectively.

If preferences are time separable, the smallest eigenvalue and the highest eigenvalue simplify as follows:

$$
\mu_{1}^{\gamma=0}=-\sigma<0, \quad \mu_{4}^{\gamma=0}=r^{\star}+\sigma>0 .
$$




\section{Eigenvectors}

Eigenvector $\omega_{2}^{i}$ writes as follows:

$$
\begin{aligned}
& \omega_{2}^{1}=\left(\frac{\sigma+\mu_{1}}{\sigma}\right)>0, \\
& \omega_{2}^{2}=\left(\frac{\sigma+\mu_{2}}{\sigma}\right)>0,
\end{aligned}
$$

Eigenvector $\omega_{3}^{i}$ writes as follows:

$$
\begin{aligned}
\omega_{3}^{1} & =\frac{\left(1-\alpha_{c}\right) p_{c}\left(\sigma+\mu_{1}\right) \mu_{1}}{\sigma\left[\mu_{1} \mu_{4}+I_{p} \tilde{p} F_{k k}\right]}>0, \\
\omega_{3}^{2} & =\frac{\left(1-\alpha_{c}\right) p_{c}\left(\sigma+\mu_{2}\right) \mu_{2}}{\sigma\left[\mu_{2} \mu_{3}+I_{p} \tilde{p} F_{k k}\right]}>0,
\end{aligned}
$$

Eigenvector $\omega_{4}^{i}$ writes as follows:

$$
\begin{aligned}
\omega_{4}^{1} & =\frac{\tilde{p} F_{k k}}{\mu_{1}} \omega_{1}^{3}>0, \\
\omega_{4}^{2} & =\frac{\tilde{p} F_{k k}}{\mu_{2}} \omega_{2}^{3}>0,
\end{aligned}
$$

where the signs of $\omega_{2}^{1}, \omega_{2}^{2}, \omega_{3}^{2}$ and $\omega_{4}^{2}$ stem from the following condition that we imposed:

$$
I_{p} \tilde{p} F_{k k}<\sigma(\sigma+\delta)
$$

with $\delta=r^{\star}$.

\section{References}

Becker, Gary S. and Kevin M. Murphy. (1988) A Theory of Rational Addiction. Journal of Political Economy 96, 675-700.

Dockner, Engelbert J. and Gustav Feichtinger (1991) On the Optimality of Limit Cycles in Dynamic Economic Systems. Journal of Economics 53(1), 31-50.

Ryder, Harl E., and Geoffrey M. Heal (1973) Optimal Growth with Intertemporally Dependent Preferences. Review of Economic Studies 40, 1-31.

Shi, Shouyong, and Larry G. Epstein (1993) Habits and Time preference. International Economic Review, 34 (1), 61-84. 